\title{
HARMONIC DIFFEOMORPHISMS BETWEEN COMPLETE RIEMANN SURFACES OF NEGATIVE CURVATURE*
}

\author{
QUN CHEN ${ }^{\dagger}$ AND JÜRGEN EICHHORN ${ }^{\ddagger}$
}

\begin{abstract}
Consider two open Riemannian surfaces $\left(M^{2}, g_{0}\right),\left(M^{2}, g_{1}\right)$, a curve $\left\{g_{t}\right\}_{0 \leq t \leq 1}$ in the Sobolev topology, $K_{g_{t}} \equiv-1$, inf $\sigma\left(\Delta_{0}\left(g_{t}\right)\right)>0, r_{i n j}\left(g_{t}\right)>0,0 \leq t \leq 1$. We prove that there exists a unique harmonic diffeomorphism $f_{1}:\left(M^{2}, g_{0}\right) \rightarrow\left(M^{2}, g_{1}\right)$ which is moreover isotopic by harmonic diffeomorphisms to id : $\left(M^{2}, g_{0}\right) \rightarrow\left(M^{2}, g_{0}\right)$ in the unit component $\mathcal{D}_{0}^{r+1}$ of the completed diffeomorphism group $\mathcal{D}^{r+1}$. This has application in Teichmüler theory for open surfaces.
\end{abstract}

Key words. Harmonic map, open surfaces, Teichmüler space, diffeomorphism.

AMS subject classifications. 58D27, 58E20

1. Introduction. In [5] the second author introduced Teichmüller spaces for open manifolds (i.e., noncompact complete manifolds) as follows. Let $\left(M^{n}, g\right)$ be an open Riemannian manifold with bounded geometry, the Sobolev completed space $\mathcal{M}^{2, r}\left(I, B_{k}\right)$ (c.f. [5]) splits into its arc components $\mathcal{M}^{2, r}\left(I, B_{k}\right)=\sum_{i \in I} \operatorname{comp} p^{2, r}\left(g_{i}\right)$.

We considered the case $n=2$, i.e. $\left(M^{2}, g_{0}\right)$ with the sectional curvature $K_{g_{0}} \equiv-1$, the lowest spectral value $\inf \sigma\left(\Delta_{0}\left(g_{0}\right)\right)>0$, the injectivity radius $r_{i n j}\left(g_{0}\right)>0$ and its arc component $\operatorname{comp}^{2, r}\left(g_{0}\right)$. We defined a completed space $\mathcal{P}^{r}\left(g_{0}\right)$ of positive conformal factors. $\mathcal{P}^{r}=\sum_{i} \operatorname{comp}\left(e^{u_{i}}\right)$, and $\operatorname{comp}(1) \subset \mathcal{P}^{r}\left(g_{0}\right)$ is an invariant of $\operatorname{comp}\left(g_{0}\right) \cdot \operatorname{comp}(1)$ acts on $\operatorname{comp}\left(g_{0}\right)$. We proved that under the above assumptions there exists for any $g \in \operatorname{comp}\left(g_{0}\right)$ a unique $e^{u} \in \operatorname{comp}(1) \subset \mathcal{P}^{r}\left(g_{0}\right)$ such that $K_{e^{u} g} \equiv$ -1 . Thereafter we defined as Teichmüller space of $c o m p^{2, r}\left(g_{0}\right)$ the space

$$
\mathcal{T}^{r}\left(\operatorname{comp}\left(g_{0}\right)\right):=\operatorname{comp}(1) \backslash \operatorname{comp}^{2, r}\left(g_{0}\right) / \mathcal{D}_{0}^{r+1} .
$$

If $M^{2}$ would be closed, then $\mathcal{M}^{2, r}\left(I, B_{k}\right)$ consists of only one component, and we come back to the classical Teichmüller space.

In the open case, we would be interested in the topological structure of $\mathcal{T}^{r}\left(\operatorname{comp}\left(g_{0}\right)\right)$. It follows from the slice theorem in [7] that $\mathcal{T}^{r}\left(\operatorname{comp}\left(g_{0}\right)\right)$ is a Hilbert manifold. One canonical method to get some insight into the topological structure is the construction of a Morse function. Assume that for any $g_{1} \in \operatorname{comp}^{2, r}\left(g_{0}\right)$ with $K_{g_{1}} \equiv-1$ there exists a unique harmonic diffeomorphism $f:\left(M^{2}, g_{0}\right) \rightarrow\left(M^{2}, g_{1}\right)$ in $\mathcal{D}_{0}^{2, r+1}$, then we prove in a forthcoming paper that in fact

$$
\phi\left(g_{1}\right):=\int_{M^{2}}\left[e\left(f:\left(M^{2}, g_{0}\right) \rightarrow\left(M^{2}, g_{1}\right)\right)-e\left(\mathrm{id}:\left(M^{2}, g_{0}\right) \rightarrow\left(M^{2}, g_{0}\right)\right)\right] d \operatorname{vol}_{x}\left(g_{0}\right),
$$

where $e(h)$ is the energy density of $h$, defines a Morse function on $\mathcal{T}^{r}\left(\operatorname{comp}\left(g_{0}\right)\right)$.

Hence we have to assure the assumption above. This is the content of this paper and our

\footnotetext{
${ }^{*}$ Received December 31, 2008; accepted for publication November 10, 2009.

${ }^{\dagger}$ School of Mathematics and Statistics, Wuhan University, Wuhan 430072, China (qunchen@ whu.edu.cn).

${ }_{\ddagger}^{\ddagger}$ Institute for Mathematics, Greifswald University, D-17487 Greifswald, Germany (eichhorn@unigreifswald.de).
} 
Main Theorem. Let $\left(M^{2}, g_{0}\right)$ be an open Riemannian surface with $K_{g_{0}} \equiv$ -1 , the injective radius $r_{i n j}\left(g_{0}\right)>0$ and the lowest spectral value of $\left(M, g_{0}\right)$ is $\inf \sigma\left(\Delta_{0}\left(g_{0}\right)\right)>0$. If $g_{1} \in \operatorname{comp}^{2, r}\left(g_{0}\right)$ and $\left\{g_{t}\right\}_{0 \leq t \leq 1}$ is a smooth curve between $g_{0}$ and $g_{1}$ such that $K_{g_{t}} \equiv-1$, then there exists a unique curve of harmonic diffeomorphisms $\left\{f_{t}:\left(M^{2}, g_{0}\right) \rightarrow\left(M^{2}, g_{t}\right)\right\}_{0 \leq t \leq 1}$, in particular, $f_{1}$ is isotopic in $\mathcal{D}_{0}^{2, r+1}=\operatorname{comp}^{2, r+1}(i d) \subset \mathcal{D}^{2, r+1}$ to id $:\left(M^{2}, g_{0}\right) \rightarrow\left(M^{2}, g_{0}\right)$.

The proof of this theorem considerably differs from existent proofs in the compact case. In the compact case one more or less uses some compactness arguments, mostly of Arzela-Ascoli type, or properties of the heat flow on compact manifolds. In the open complete case, one has to establish another approach. Very successful approaches are e.g. the papers [3], [18],[19],[27],[31]. Our approach here essentially relies on the framework of nonlinear Sobolev analysis on open manifolds established in [12].

The paper is organized as follows. In section 2 we collect those constructions and facts of linear and nonlinear Sobolev analysis on open manifolds which are needed in the sequel. This contains Sobolev spaces, spaces of Riemannian metrics, manifolds of maps and diffeomorphism groups. Section 3 is devoted to harmonic maps as stationary points of a relative energy functional. In section 4 we present gradient estimates for harmonic maps between open surfaces, which are of extraordinarily importance for the above sections. We prove in section 5 by means of standard techniques that locally we can extend a curve of harmonic $f_{t}:\left(M, g_{0}\right) \rightarrow\left(M, g_{t}\right)$. In section 6 , we reduce our main theorem to the Banach fixed point theorem. The section 7 contains the proof of the existence theorem. It amounts to quite formidable estimates. The concluding section 8 contains the uniqueness and the proof of the main theorem, i.e., the proof that the harmonic maps in question are in fact diffeomorphisms. We would like to point out that the open manifolds considered here and in [5] are all of infinite genus.

There are other existence theorems, we refer to [19], [27] and [31]. One of the special features of our approach is the fact that we work in Sobolev category, we reduce to the Banach fixed point theorem, and we obtain harmonic diffeomorphisms and a whole curve of such diffeomorphisms.

Another approach for open complete surfaces has been established by J. Lohkamp in [20] and [21]. M. Wolf used in [29] harmonic diffeomorphisms to describe the Teichmüller space of compact surfaces and Wolf-Weber in [30] used Teichmüller theory to construct complete minimal surfaces of finite genus.

In [20] Lohkamp proved the following

Theorem ([20]). Let $f: M \rightarrow N$ be simple. Then there exists a harmonic map homotopic to $f$.

We refer to [20] concerning the exact definition of simple. Roughly speaking, it means that there exists an exhaustion $M_{i}$ by compact submanifolds such that the energy of $\mathrm{f}$ restricted to the annulus $M_{i+1} / M_{i}$ becomes very small . J. Lohkamp gives classes of examples for simple maps. One class (he uses) are maps $f: M \rightarrow N$ with $M$ a punctured closed Riemann surface. The proof of the theorem essentially uses some kinds of compactness arguments, whose applicability comes from the simplicity of $f$ (at the ends = cusps the injectivity radius tends to zero, the volume of a cusp is finite etc.). In our case, the initial map $i d:\left(M, g_{0}\right) \rightarrow\left(M, g_{0}\right)$ or $f_{t}:\left(M, g_{0}\right) \rightarrow\left(M, g_{t}\right)$ is not simple, and the arguments of Lohkamp are not applicable.

In [21] Lohkamp proved the following 
TheOREM ([21], p.343). If $f: X \rightarrow Y$ is a homeomorphism between punctured surfaces, then there exists a unique harmonic map $h$ homotopic to $f$ with $E(h)<\infty$. $h$ is a diffeomorphism.

Here the author again uses an exhaustion argument adapted to the special structure of the ends. We can not see the possibility of generalization of these arguments to the case of infinitely connected ends. In the case of cusp manifolds, one can traditionally separate the considerations, one considers the contribution of the compact part and the contribution of the cusps. In our case, such a separation is impossible.

Hence, our results and those of Lohkamp and Wolf are disjoint, since the classes under consideration are completely disjoint.

The authors are deeply indebted to the Deutsche Forschungsgemeinschaft and the NSF of China for the support of their collaboration. One third of the paper has been established at the Greifswald University, the second third at the Wuhan University and the last third at the MPI for Mathematics in Bonn. The research of QC was partially supported by NSFC (Nos.10571068, 10871149) and RFDP (No.200804860046). The authors are deeply indebted to these institutions.

2. Nonlinear Sobolev analysis. In this section, we briefly collect those facts from nonlinear Sobolev analysis which we essentially need in the sequel. We restrict to bounded geometry and say $\left(M^{n}, g\right)$ has bounded geometry up to order $k$ if it satisfies the condition $(I)$ and $\left(B_{k}(M, g)\right)$ :

$$
\begin{gathered}
r_{i n j}(M, g)>0 \\
\left|\nabla^{i} R\right| \leq C_{i}, \forall i=0,1, \cdots, k .
\end{gathered}
$$

The condition $\left(B_{\infty}(M, g)\right)$ means $\left|\nabla^{i} R\right| \leq C_{i}, i=1,2, \cdots$. Every closed Riemannian manifold satisfies $(I)$ and $\left(B_{\infty}(M, g)\right)$. Examples of open manifolds satisfying $(I)$ and $\left(B_{\infty}(M, g)\right)$ are homogeneous spaces or Riemannian coverings of closed manifolds. Greene has proven that every open manifold admits a metric $g$ satisfying $(I)$ and $\left(B_{\infty}(M, g)\right)$, i.e., bounded geometry does not affect the topological type. We restrict in most of our considerations to bounded geometry. The reason for this is the fact that then Sobolev analysis is available, e.g. embedding theorems, module structure theorems and many invariance properties. If we give up $(I)$ for instance, then these theorems do not apply. Parts of them still hold by using weighted Sobolev spaces, but this requires additional effort. We list some important consequences of $(I)$ and $\left(B_{k}\right)$.

Proposition 2.1. a) (I) implies completeness of $\left(M^{n}, g\right)$.

b) If $\left(M^{n}, g\right)$ satisfies $(I)$ and $\left(B_{k}\right)$ and $\mathscr{U}=\left\{\left(U_{\alpha}, \Phi_{\alpha}\right)\right\}_{\alpha}$ is a locally finite cover by normal charts, then there exist constants $C_{\beta}, C_{\beta}^{\prime}, C_{\gamma}^{\prime}$, multi-indexed by $\beta, \gamma$ such that

$$
\left|D^{\beta} g_{i j}\right| \leq C_{\beta}, \quad\left|D^{\beta} g^{i j}\right| \leq C_{\beta}^{\prime}, \quad \text { for } \quad|\beta| \leq k
$$

and

$$
\left|D^{\gamma} \Gamma_{i j}^{m}\right| \leq C_{\gamma}^{\prime}, \quad|\gamma| \leq k-1,
$$

all constants are independent of $\alpha$. 
c) If $\left(E, h, \nabla^{E}\right) \rightarrow\left(M^{n}, g\right)$ is a Riemannian vector bundle satisfying $(I)$ and $\left(B_{k}(M, g)\right),\left(B_{k}(E, \nabla)\right)$, then additionally to (2.1), (2.2) there holds for the connection coefficients $\Gamma_{i \lambda}^{\mu}$ defined by $\nabla_{\frac{\partial}{\partial u^{i}}} \phi_{\lambda}=\Gamma_{i \lambda}^{\mu} \phi_{\mu},\left\{\phi_{\mu}\right\}_{\mu}$ a local orthonormal frame obtained by radial parallel translation,

$$
\left|D^{\beta} \Gamma_{i \lambda}^{\mu}\right| \leq D_{\beta}, \quad|\beta| \leq k-1 .
$$

Proof. Under the assumption of $(I)$ any Cauchy sequence $\left(x_{\nu}\right)_{\nu}$ in $M$ can be considered, up to quasi-isometry, as contained in a small closed Euclidean ball, omitting only a finite number of the $x_{\nu}$ 's. This proves a). b) and c) are the content of [10].

Proposition 2.2. Assume $\left(M^{n}, g\right)$ satisfies (I) and ( $\left.B_{0}\right)$. There exists $\varepsilon_{0}>0$ such that for any $\varepsilon \in\left(0, \varepsilon_{0}\right)$ there is a countable cover of $M$ by geodesic balls $B_{\varepsilon}\left(x_{i}\right)$, $\cup_{i} B_{\varepsilon}\left(x_{i}\right)=M$, such that the cover of $M$ by the balls $B_{2 \varepsilon}\left(x_{i}\right)$ with double radius and same centers is still uniformly locally finite.

We refer to [12] for the proof. Proposition 2.2 implies the existence of an associated uniform partition of unity.

Proposition 2.3. Assume $\left(M^{n}, g\right)$ open with $(I)$ and $\left(B_{k}\right)$ for $r \in\left(0, r_{i n j}\right)$. For every $0<\varepsilon<r / 2$ there exists a partition of unity $1=\sum_{i=1}^{\infty} \psi_{i}$ on $M$ such that

1) $\psi_{i} \geq 0, \psi_{i} \in C_{c}^{\infty}(M), \operatorname{supp} \psi_{i} \subset B_{2 \varepsilon}\left(x_{i}\right)$, where the sequence $\left\{x_{i}\right\}_{i}$ comes from Proposition 2.2.

2) $\left|D_{u}^{\beta} \psi_{i}\left(u^{1}, \cdots, u^{n}\right)\right| \leq C_{\beta},|\beta| \leq k+2$, where $\left(u^{1}, \cdots, u^{n}\right)$ are normal coordinates in $B_{2 \varepsilon}\left(x_{i}\right)$.

We refer to[12] for the proof.

Let $\left(E, h, \nabla^{h}\right) \rightarrow\left(M^{n}, g\right)$ be a Riemannian vector bundle. Then the Levi-Civita connection $\nabla^{g}$ and $\nabla^{h}$ define metric connections $\nabla$ in all tensor bundles $T_{v}^{u} \otimes E$. Denote smooth sections as above by $C^{\infty}\left(T_{v}^{u} \otimes E\right)$, and by $C_{c}^{\infty}\left(T_{v}^{u} \otimes E\right)$ those with compact support. In the sequel we shall write $E$ instead of $T_{v}^{u} \otimes E$, keeping in mind that $E$ can be an arbitrary vector bundle. Now we define for $p \in \mathbb{R}, 1 \leq p<\infty$ and $r$ a non-negative integer

$$
\begin{gathered}
|\phi|_{p, r}:=\left(\int \sum_{i=0}^{r}\left|\nabla^{i} \phi\right|_{x}^{p} d \operatorname{vol}_{x}(g)\right)^{1 / p}, \\
\Omega_{r}^{0, p}(E) \equiv \Omega_{r}^{p}(E)=\left\{\left.\phi \in C^{\infty}(E)|| \phi\right|_{p, r}<\infty\right\}, \\
\bar{\Omega}^{0, p, r}(E) \equiv \bar{\Omega}^{p, r}(E)=\text { completion of } \Omega_{r}^{p}(E) \text { with respect to }|\cdot|_{p, r}, \\
\stackrel{\circ}{\Omega^{0, p, r}}(E) \equiv \stackrel{\circ}{\Omega}^{p, r}(E)=\text { completion of } C_{c}^{\infty}(E) \text { with respect to }|\cdot|_{p, r}, \\
\Omega^{0, p, r}(E) \equiv \Omega^{p, r}(E)=\left\{\phi \mid \quad \phi \text { measurable distribution section with }|\phi|_{p, r}<\infty\right\} .
\end{gathered}
$$


Here we use the standard identification of sections of a vector bundle $E$ with $E$-valued zero-forms. $\Omega^{q, p, r}(E)$ stands for a Sobolev space of $q$-forms with values in E.

For $p=2$, we often use the notations $|\cdot|_{2,0}=|\cdot|_{L_{2}}$. Furthermore, we define

$$
\begin{gathered}
{ }^{b, m}|\phi|:=\sum_{i=0}^{m}\left|\nabla^{i} \phi\right|_{x}, \\
{ }^{b, m} \Omega(E)=\left\{\phi \mid \quad \phi \quad C^{m}-\text { section and }{ }^{b, m}|\phi|<\infty\right\}, \\
{ }^{b, m} \stackrel{\circ}{\Omega}(E)=\text { completion of } C_{c}^{\infty}(E) \text { with respect to }{ }^{b, m}|\cdot|,
\end{gathered}
$$

${ }^{b, m} \Omega(E)$ equals the completion of

$$
{ }_{m}^{b} \Omega(E)=\left\{\left.\phi \in C^{\infty}(E)\right|^{b, m}|\phi|<\infty\right\}
$$

with respect to ${ }^{b, m}|\cdot|$.

Denote by ${ }^{b, \infty} \Omega(E)$ the locally convex space of smooth sections $\phi$ such that $\nabla^{s} \phi$ is bounded for $s=0,1,2, \cdots$.

Proposition 2.4. The spaces $\stackrel{\circ}{\Omega}^{p, r}(E), \bar{\Omega}^{p, r}(E), \Omega^{p, r}(E),{ }^{b, m} \stackrel{\circ}{\Omega}(E),{ }^{b, m} \Omega(E)$ are Banach spaces and there are inclusions

$$
\begin{gathered}
\stackrel{\circ}{\Omega}^{p, r}(E) \subseteq \bar{\Omega}^{p, r}(E) \subseteq \Omega^{p, r}(E), \\
{ }^{b, m} \stackrel{\circ}{\Omega}(E) \subseteq \subseteq^{b, m} \Omega(E) .
\end{gathered}
$$

If $p=2$, then $\stackrel{\circ}{\Omega^{2, r}}(E), \bar{\Omega}^{2, r}(E), \Omega^{2, r}(E)$ are Hilbert spaces.

In general, $\stackrel{\circ}{\Omega^{p, r}}(E), \bar{\Omega}^{p, r}(E), \Omega^{p, r}(E)$ could be different from one another.

Proposition 2.5. If $\left(M^{n}, g\right)$ satisfies $(I)$ and $\left(B_{k}\right)$, then

$$
\stackrel{\circ}{\Omega}^{p, r}(E)=\bar{\Omega}^{p, r}(E)=\Omega^{p, r}(E), \quad 0 \leq r \leq k+2 .
$$

Proof. We refer to [12] for the proof.

Embedding theorems are of great importance in non-linear global analysis and even more the module structure theorem which we present now.

Theorem 2.6. a) Assume $r-\frac{n}{p} \geq s-\frac{n}{q}, r \geq s$. Let $B \subset \mathbb{R}^{n}$ be a Euclidean ball. Then

$$
\stackrel{\circ}{\Omega}^{p, r}\left(B \times \mathbb{R}^{n}\right) \hookrightarrow \stackrel{\circ}{\Omega}^{q, s}\left(B \times \mathbb{R}^{n}\right)
$$

continuously. 
b) If $r-\frac{n}{p} \geq s, s \in \mathbb{Z}_{+}$, then

$$
\stackrel{\circ}{\Omega}^{p, r}\left(B \times \mathbb{R}^{n}\right) \hookrightarrow \stackrel{\circ}{\Omega}^{q, s}\left(B \times \mathbb{R}^{n}\right)
$$

continuously.

The global version of a) looks slightly different.

TheOrem 2.7. Let $\left(E, h, \nabla^{E}\right) \rightarrow\left(M^{n}, g\right)$ be a Riemannian vector bundle satisfying $(I),\left(B_{k}\left(M^{n}, g\right)\right),\left(B_{k}(E, \nabla)\right), k \geq 1$.

a) Assume $r-\frac{n}{p} \geq s-\frac{n}{q}, r \geq s, q \geq p$. Then

$$
\Omega^{p, r}(E) \hookrightarrow \Omega^{q, s}(E)
$$

continuously.

b) If $r-\frac{n}{p} \geq s, s \in \mathbb{Z}_{+}$, then

$$
\Omega^{p, r}(E) \hookrightarrow \Omega^{b, s}(E)
$$

continuously.

We refer to [11] for a proof.

Now we come to the module structure theorem.

ThEOREM 2.8. Let $\left(E_{i}, h_{i}, \nabla_{i}\right) \rightarrow\left(M^{n}, g\right)$ be Riemannian vector bundles satisfying $(I),\left(B_{k}\left(M^{n}, g\right)\right),\left(B_{k}\left(E_{i}, \nabla_{i}\right)\right), i=1,2$. Assume $0 \leq r \leq r_{1}, r_{2} \leq k$. If $r=0$ assume

$$
\begin{gathered}
\left\{\begin{array}{c}
r-\frac{n}{p}<r_{1}-\frac{n_{1}}{p_{1}} \\
r-\frac{n}{p}<r_{2}-\frac{n_{2}}{p_{2}} \\
r-\frac{n}{p} \leq r_{1}-\frac{n_{1}}{p_{1}}+r_{2}-\frac{n_{2}}{p_{2}} \\
\frac{1}{p} \leq \frac{1}{p_{1}}+\frac{1}{p_{2}}
\end{array}\right\} \text { or } \\
\left\{\begin{array}{c}
r-\frac{n}{p} \leq r_{1}-\frac{n_{1}}{p_{1}} \\
0<r_{2}-\frac{n_{2}}{p_{2}} \\
\frac{1}{p} \leq \frac{1}{p_{1}}
\end{array}\right\} \text { or }\left\{\begin{array}{c}
0<r_{1}-\frac{n_{1}}{p_{1}} \\
r-\frac{n}{p} \leq r_{2}-\frac{n_{2}}{p_{2}} \\
\frac{1}{p} \leq \frac{1}{p_{2}}
\end{array}\right\} .
\end{gathered}
$$

If $r>0$, assume $\frac{1}{p} \leq \frac{1}{p_{1}}+\frac{1}{p_{2}}$ and

$$
\left\{\begin{array}{c}
r-\frac{n}{p}<r_{1}-\frac{n_{1}}{p_{1}} \\
r-\frac{n}{p}<r_{2}-\frac{n_{2}}{p_{2}} \\
r-\frac{n}{p} \leq r_{1}-\frac{n_{1}}{p_{1}}+r_{2}-\frac{n_{2}}{p_{2}}
\end{array}\right\} \text { or }\left\{\begin{array}{c}
r-\frac{n}{p} \leq r_{1}-\frac{n_{1}}{p_{1}} \\
r-\frac{n}{p} \leq r_{2}-\frac{n_{2}}{p_{2}} \\
r-\frac{n}{p}<r_{1}-\frac{n_{1}}{p_{1}}+r_{2}-\frac{n_{2}}{p_{2}}
\end{array}\right\} .
$$

Then the tensor product of sections defines a continuous bilinear map

$$
\Omega^{p_{1}, r_{1}}\left(E_{1}, \nabla_{1}\right) \times \Omega^{p_{2}, r_{2}}\left(E_{2}, \nabla_{2}\right) \rightarrow \Omega^{p, r}\left(E_{1} \otimes E_{2}, \nabla_{1} \otimes \nabla_{2}\right) .
$$

We refer to [12] for the proof. $\square$

Corollary 2.9. Assume $r=r_{1}=r_{2} \cdot p=p_{1}=p_{2}$.

(a) If $E_{1}=M \times \mathbb{R}, E_{2}=E$, then $\Omega^{p, r}(E)$ is an $\Omega^{p, r}(M \times \mathbb{R})$-module. 
(b) If $E_{1}=M \times \mathbb{R}=E_{2}$, then $\Omega^{p, r}(M \times \mathbb{R})$ is a commutative, associative Banach algebra.

(c) If $E_{1}=E=E_{2}$, then the tensor product of sections defines a continuous map

$$
\Omega^{p, r}(E) \times \Omega^{p, r}(E) \rightarrow \Omega^{p, r}(E \otimes E) .
$$

Given $\left(E, h, \nabla^{E}\right) \rightarrow\left(M^{n}, g\right)$, for fixed $E \rightarrow M, r \geq 0, p \geq 1$, the Sobolev space $\Omega^{p, r}\left(E, h, \nabla^{E}, g, \nabla^{g}, d \operatorname{vol}_{x}(g)\right)$ depends on $h, \nabla=\nabla^{E}$, and $g$. Moreover, if we choose another sequence of differential operators with injective symbol, e.g. $D, D^{2}, \cdots$ in case of a Clifford bundle, we should get other Sobolev spaces. Hence two questions arise, namely

1) the dependence on the choice of $h, \nabla^{E}, g$,

2) the dependence on the sequence of differential operators.

We start with the first issue and investigate the dependence on the metric connection $\nabla=\nabla^{E}$ of $(E, h)$. If $\nabla^{\prime}=\nabla^{\prime E}$ is another metric connection then $\eta=\nabla^{\prime}-\nabla$ is a 1-form with values in $\mathfrak{G}_{E}, \nabla^{\prime}-\nabla \in \Omega^{1}\left(\mathfrak{G}_{E}\right)=\Omega\left(T^{*} M \otimes \mathfrak{G}_{E}\right)$. Here $\mathfrak{G}_{E}$ is the bundle of the skew-symmetric endomorphisms. $\nabla=\nabla^{E}$ induces a connection $\nabla=\nabla^{\mathfrak{G}_{E}}$ in $\mathfrak{G}_{E}$ and hence a Sobolev norm $\left|\nabla^{\prime}-\nabla\right|_{\nabla, p, r}=\left|\nabla^{\prime}-\nabla\right|_{h, \nabla, g, \nabla^{g}, p, r}$.

TheOREM 2.10. Assume $\left(E, h, \nabla^{E}\right) \rightarrow\left(M^{n}, g\right)$ be a Riemannian vector bundle satisfying $(I),\left(B_{k}\left(M^{n}, g\right)\right),\left(B_{k}\left(E, \nabla^{E}\right)\right), k \geq r>\frac{n}{q}+1$. Let $\nabla^{\prime}=\nabla^{E}$ be a second metric connection with $\left.B_{k}\left(E, \nabla^{\prime E}\right)\right)$ and suppose

$$
\left|\nabla-\nabla^{\prime}\right|_{\nabla, p, r-1}<\infty \text {. }
$$

Then

$$
\Omega^{p . \rho}(E, h, \nabla, g)=\Omega^{p \cdot \rho}\left(E, h, \nabla^{\prime}, g\right), \quad 0 \leq \rho \leq r
$$

as Sobolev spaces.

This can be extended to a more general

TheOREM 2.11. Let $\left(E, h, \nabla^{E}\right) \rightarrow\left(M^{n}, g\right)$ be a Riemannian vector bundle satisfying $(I),\left(B_{k}\left(M^{n}, g\right)\right),\left(B_{k}\left(E, \nabla^{E}\right)\right), k \geq r>\frac{n}{q}+1$. Suppose $h^{\prime}$ is a fibre metric on $E$ with metric connection $\nabla^{\prime}$ and $g^{\prime}$ a metric on $M^{n}$ with $(I),\left(B_{k}\left(M^{n}, g^{\prime}\right)\right)$, $\left(B_{k}\left(E, \nabla^{\prime}\right)\right)$ satisfying $C \dot{h} \leq h^{\prime} \leq D \cdot h, C_{1} \cdot g \leq g^{\prime} \leq C_{2} \cdot g,\left|\nabla-\nabla^{\prime}\right|_{h, \nabla, g, p, r-1}<\infty$, $\left|\nabla^{g^{\prime}}-\nabla^{g}\right|_{g, p, r-1}<\infty$. then

$$
\Omega^{p, \rho}(E, h, \nabla, g)=\Omega^{p, \rho}\left(E, h^{\prime}, \nabla^{\prime}, g^{\prime}\right), \quad 0 \leq \rho \leq r
$$

as equivalent Sobolev spaces.

We are left with the dependence on the sequence of differential operators. This can be answered by the following two theorems.

TheOrem 2.12. Let $\left(M^{n}, g\right)$ be an open Riemannian manifold satisfying $\left(B_{\infty}\left(M^{n}, g\right)\right)$. Then

$$
\stackrel{\circ}{\Omega}^{q, 2,2 s}(M, \nabla)=\stackrel{\circ}{\Omega}^{q, 2,2 s}(M, \Delta), \quad 0 \leq q \leq n, \quad, s=0,1,2, \cdots
$$


as equivalence of Sobolev spaces.

Here the $\Omega^{\prime} s$ are Sobolev space of forms.

TheOrem 2.13. Let $\left(E, h, \nabla^{E}\right) \rightarrow\left(M^{n}, g\right)$ be a Clifford bundle satisfying $\left(B_{\infty}\left(M^{n}, g\right)\right)$ and $\left(B_{\infty}(E, \nabla)\right)$. If $\left(M^{n}, g\right)$ is complete, then

$$
\Omega^{2, r}(E, \nabla)=\Omega^{2, r}(E, D), \quad, r=0,1,2, \cdots
$$

as equivalence of Sobolev spaces.

We refer to [12] for the proof.

For later use we restric ourselfs additionally to metrics with bounded geometry. Let $\left(M^{n}, g\right)$ be open. Consider the conditions $(I)$ and $\left(B_{k}\right)$ and

$$
\begin{gathered}
\mathcal{M}(I)=\{g \in \mathcal{M} \mid \quad g \quad \text { satisfies } \quad(I)\}, \\
\mathcal{M}\left(B_{k}\right)=\left\{g \in \mathcal{M} \mid \quad g \quad \text { satisfies } \quad\left(B_{k}\right)\right\} \\
\mathcal{M}\left(I, B_{k}\right)=\mathcal{M}(I) \cap \mathcal{M}\left(B_{k}\right) .
\end{gathered}
$$

Now we want to introduce Sobolev uniform structures into the space of metrics. Let now $k \geq r>\frac{n}{p}+1, \delta>0, C(n, \delta)=1+\delta+\delta \sqrt{2 n(n-1)}$,

$$
\begin{aligned}
V_{\delta}= & \left\{\left(g, g^{\prime}\right) \in \mathcal{M}\left(I, B_{k}\right) \times \mathcal{M}\left(I, B_{k}\right) \mid C(n, \delta)^{-1} g \leq g^{\prime} \leq C(n, \delta) g,\right. \\
& \left|g-g^{\prime}\right|_{g, p, r}=\left(\int\left(\left|g-g^{\prime}\right|_{g, x}^{p}+\sum_{i=0}^{r-1}\left|\left(\nabla^{g}\right)^{i}\left(\nabla^{g^{\prime}}-\nabla^{g}\right)\right|_{g, x}^{p} d \operatorname{vol}_{x}(g)\right)^{\frac{1}{p}}<\delta\right\} .
\end{aligned}
$$

Proposition 2.14. The set $\left\{V_{\delta}\right\}_{\delta>0}$ is a basis for a metrizable uniform structure $\left.\mathfrak{U}^{p, r}\left(\mathcal{M}\left(I, B_{k}\right)\right)\right)$ on $\mathcal{M}\left(I, B_{k}\right)$.

Denote $\mathcal{M}_{r}^{P}\left(I, B_{k}\right)$ as $\left(\mathcal{M}\left(I, B_{k}\right), \mathfrak{U}^{p, r}\left(\mathcal{M}\left(I, B_{k}\right)\right)\right)$ and by $\mathcal{M}^{p, r}\left(I, B_{k}\right)$ the completion. It was proven by Salomonsen that the completion yields only positive definite elements, i.e. we still remain in the space of $C^{1}$ Riemannian metrics.

For $g \in \mathcal{M}^{p, r}\left(I, B_{k}\right)$,

$$
\begin{aligned}
\left\{U_{\varepsilon}^{p, r}(g)\right\}_{\varepsilon>0}= & \left\{\left\{g^{\prime} \in \mathcal{M}^{p, r}\left(I, B_{k}\right)\left|{ }^{b}\right| g-\left.g^{\prime}\right|_{g}<\varepsilon, \quad{ }^{b}\left|g-g^{\prime}\right|_{g^{\prime}}<\varepsilon,\right.\right. \\
& \left.\left.\left|g-g^{\prime}\right|_{g, p, r}<\varepsilon\right\}\right\}_{\varepsilon>0}
\end{aligned}
$$

is a neighborhood basis in the uniform topology. There arises a small difficulty. $g \in \mathcal{M}^{p, r}\left(I, B_{k}\right)$ must not be smooth and hence $\left|g-g^{\prime}\right|_{g, p, r}$ must not be defined immediately. But in this case we use the density of $\mathcal{M}\left(I, B_{k}\right) \subset \mathcal{M}^{p, r}\left(I, B_{k}\right)$ and apply a suitable approximations procedure (cf. [12]).

Proposition 2.15. The space $\mathcal{M}^{p, r}\left(I, B_{k}\right)$ is locally contractible.

Proposition 2.16. In $\mathcal{M}^{p, r}\left(I, B_{k}\right)$ components and arc components coincide. 
Set for $g \in \mathcal{M}^{p, r}\left(I, B_{k}\right)$

$$
\begin{aligned}
U^{p, r}(g)= & \left\{g^{\prime} \in \mathcal{M}^{p, r}\left(I, B_{k}\right)\left|{ }^{b}\right| g-\left.g^{\prime}\right|_{g}<\infty, \quad{ }^{b}\left|g-g^{\prime}\right|_{g^{\prime}}<\infty,\right. \\
& \left.\left.\left|g-g^{\prime}\right|_{g, p, r}<\infty\right\}\right\} .
\end{aligned}
$$

Proposition 2.17. Denote by comp $(g)$ the component of $g \in \mathcal{M}^{p, r}\left(I, B_{k}\right)$. Then

$$
\operatorname{comp}(g) \equiv \operatorname{comp}^{p, r}(g)=U^{p, r}(g) .
$$

Theorem 2.18. Let $\left(M^{n}\right.$ be open, $k \geq r>\frac{n}{p}+1$. Then $\mathcal{M}^{p, r}\left(I, B_{k}\right)$ has a representation as a topological sum

$$
\mathcal{M}^{p, r}\left(I, B_{k}\right)=\sum_{i \in I} U^{p, r}\left(g_{i}\right)
$$

We can reformulate theorems 2.10 and 2.11 .

Proposition 2.19. Let $g \in \mathcal{M}\left(I, B_{k}\right), k \geq r>\frac{n}{p}+1, g^{\prime} \in \operatorname{comp}(g) \subset$ $\mathcal{M}^{p, r}\left(I, B_{k}\right)$. Then

$$
\Omega^{p, r}\left(T_{v}^{u}, g\right)=\Omega^{p, r}\left(T_{v}^{u}, g^{\prime}\right)
$$

as equivalence of Sobolev spaces.

Theorem 2.20. Assume $k \geq r>\frac{n}{p}+1$. Then, each component of the space $\mathcal{M}^{p, r}\left(I, B_{k}\right)$ is a Banach manifold and for $p=2$ it is a Hilbert manifold.

We introduce now in quite analogous manner uniform structures of connections. Let $(E, h) \rightarrow\left(M^{n}, g\right)$ be a Riemannian vector bundle. Denote by $\mathcal{C}_{E}$ the set of all metric connections in $E$ and set for $m \in \mathbb{Z}_{+}, \delta>0$

$$
V_{\delta}=\left\{\left.\left(\nabla, \nabla^{\prime}\right) \in \mathcal{C}_{E}^{2}\right|^{b, m}\left|\nabla^{\prime}-\nabla\right|_{\nabla}<\delta\right\},
$$

where, according to our definitions in section 2,

$$
{ }^{b, m}\left|\nabla^{\prime}-\nabla\right|_{\nabla}=\sum_{\mu=0}^{m} \sup _{x \in M}\left|\nabla^{\mu}\left(\nabla^{\prime}-\nabla\right)\right|_{x} \sim \sup _{\substack{x \in M \\ 0 \leq \mu \leq m}}\left|\nabla^{\mu}\left(\nabla^{\prime}-\nabla\right)\right|_{x} .
$$

Proposition 2.21. $\mathfrak{B}=\left\{V_{\delta}\right\}_{\delta>0}$ is a basis for a metrizable uniform structure ${ }^{b, m} \mathfrak{U}\left(\mathcal{C}_{E}\right)$ on $\mathcal{C}_{E}$.

Denote ${ }_{m}^{b} \mathcal{C}_{E}=\left(\mathcal{C}_{E},{ }^{b, m} \mathfrak{U}\left(\mathcal{C}_{E}\right)\right)$ and by ${ }^{b, m} \mathcal{C}_{E}$ the completion.

Proposition 2.22. a) ${ }^{b, m} \mathcal{C}_{E}$ is locally arcwise connected, hence components coincide with arc components.

b) ${ }^{b, m} \mathcal{C}_{E}$ has a representation as topological sum

$$
{ }^{b, m} \mathcal{C}_{E}=\sum_{i \in I}{ }^{b, m} \operatorname{comp}\left(\nabla_{i}\right) .
$$


c) For $\nabla \in^{b, m} \mathcal{C}_{E}$

$$
{ }^{b, m} \operatorname{comp}(\nabla)=\left\{\left.\nabla^{\prime} \in{ }^{b, m} \mathcal{C}_{E}\right|^{b, m}\left|\nabla^{\prime}-\nabla\right|_{\nabla}<\infty\right\}=\nabla+{ }^{b, 1} \Omega^{1}\left(\mathcal{G}_{E}\right),
$$

where $\mathcal{G}_{E}$ are the skew symmetric endomorphisms of $E$ and the connection in $\mathcal{G}_{E}$ is defined by $\nabla^{\mathcal{G}} \phi=\left[\nabla^{E}, \phi\right]$.

REMARK 2.23. On a compact manifold we have only one component.

Suppose that $\left(M^{n}, g\right)$ satisfies $\left(B_{k}\right)$ and consider the set $\mathcal{C}_{E}\left(B_{k}\right)=\{\nabla \in$ $\mathcal{C}_{E} \mid(E, \nabla)$ satisfies $\left.\left(B_{k}\right)\right\}$. Restricting ${ }^{b, m} \mathfrak{U}$ to $\mathcal{C}_{E}\left(B_{k}\right)$ by yields ${ }_{m}^{b} \mathcal{C}\left(B_{k}\right)$ and the completion ${ }^{b, m} \mathcal{C}_{E}\left(B_{k}\right)$.

Proposition 2.24. Suppose $m \geq k+1$.

a) ${ }^{b, m} \mathcal{C}_{E}\left(B_{k}\right)$ is locally arcwise connected, hence components coincide with arc components.

b) ${ }^{b, m} \mathcal{C}_{E}\left(B_{k}\right)$ has a representation as topological sum

$$
{ }^{b, m} \mathcal{C}_{E}\left(B_{k}\right)=\sum_{j \in J}{ }^{b, m} \operatorname{comp}\left(\nabla_{j}\right)
$$

We discuss another example, which is important in Teichmüller theory for open surfaces. That is the space of bounded conformal factors, adapted to a Riemannian metric $g$.

Let

$$
\mathcal{P}_{m}(g)=\left\{\left.\phi \in C^{\infty}(M)\left|\inf _{x \in M} \phi(x)>0, \sup _{x \in M} \phi(x)<\infty, \quad\right| \nabla^{i} \phi\right|_{g, x} \leq C_{i}, \quad 0 \leq i \leq m\right\} .
$$

Set for $1 \leq p<\infty, r \in \mathbb{Z}_{+}, \delta>0$,

$$
V_{\delta}=\left\{\left(\phi, \phi^{\prime}\right) \in \mathcal{P}_{m}(g)^{2}|\quad| \phi-\left.\phi^{\prime}\right|_{g, p, r}:=\left(\int \sum_{i=0}^{r}\left|\left(\nabla^{g}\right)^{i}\left(\phi-\phi^{\prime}\right)\right|_{g, x}^{p} d \operatorname{vol}_{x}(g)\right)^{\frac{1}{p}}<\delta\right\} .
$$

Then $\mathfrak{B}=\left\{V_{\delta}\right\}_{\delta>0}$ is a basis for a metrizable uniform structure.

Let $\overline{\mathcal{P}}_{m, r}^{p}(g)$ be the completion,

$$
\mathcal{C}^{1} \mathcal{P}=\left\{\phi \in C^{1}(M) \mid \inf _{x \in M} \phi(x)>0, \quad \sup _{x \in M} \phi(x)<\infty\right\}
$$

and set

$$
\mathcal{P}_{m}^{p, r}(g)=\overline{\mathcal{P}}_{m, r}^{p}(g) \cap \mathcal{C}^{1} \mathcal{P} .
$$

$\mathcal{P}_{m}^{p, r}(g)$ is locally contractible, hence locally arcwise connected and hence components coincide with arc components. Let

$$
U_{m}^{p, r}(\phi)=\left\{\phi^{\prime} \in \mathcal{P}_{m}^{p, r}(g)|\quad| \phi-\left.\phi^{\prime}\right|_{g, p, r}<\infty\right\}
$$

and denote by $\operatorname{comp}(\phi)=\operatorname{comp}_{m}^{p, r}(\phi)$ the component of $\phi$ in $\mathcal{P}_{m}^{p, r}(g) \cdot|\cdot|_{g, p, r}$ in (2.7) means the local extended metric, i.e. it is defined by taking distributional derivatives. 
TheOREM 2.25. $\mathcal{P}_{m}^{p, r}(g)$ has a representation as topological sum

$$
\mathcal{P}_{m}^{p, r}(g)=\sum_{i \in I} \operatorname{comp}\left(\phi_{i}\right)
$$

and

$$
\operatorname{comp}(\phi)=U_{m}^{p, r}(\phi) .
$$

REMARK 2.26. On a compact manifold there is only one component, the component $\operatorname{comp}(1)$.

Let $M^{n}$ be an open smooth manifold, $\mathcal{M}=\mathcal{M}(M)$ be the space of all Riemannian metrics. We want to endow $\mathcal{M}$ with a canonical intrinsic topology either in the $C^{m}-$ or Sobolev setting, depending on the subsequent investigation.

Our last class of examples for non-linear Sobolev structures are manifolds of maps and diffeomorphism groups.

Let $\left(M^{n}, g\right),\left(N^{n}, h\right)$ be open, complete, satisfying $(I)$ and $\left(B_{k}\right)$ and let $f \in$ $C^{\infty}(M, N)$. Then the differential $f_{*}=d f$ is a section of $T^{*} M \otimes f^{*} T N . f^{*} T N$ is endowed with the induced connection $f^{*} \nabla^{h}$ which is locally given by

$$
\Gamma_{i \mu}^{\nu}=\partial_{i} f^{\alpha}(x) \Gamma_{\alpha, \mu}^{h, \nu}(f(x)), \quad \partial_{i}=\frac{\partial}{\partial x^{i}} .
$$

$\nabla^{g}$ and $f^{*} \nabla^{h}$ induce metric connections $\nabla$ in all tensor bundles $T_{s}^{q}(M) \otimes f^{*} T_{v}^{u}(N)$. Therefore $\nabla^{m} d f$ is well defined. Since $(I)$ and $\left(B_{0}\right)$ imply the boundedness of the $g_{i j}, g^{k m}, h_{\mu \nu}$ in normal coordinates, the conditions $d f$ to be bounded and $\partial_{i}$ to be bounded are equivalent.

In local coordinates

$$
\sup _{x \in M}|d f|_{x}^{2}=\sup t r_{g}\left(f^{*} h\right)=\sup g^{i j} h_{\mu \nu} \partial_{j} f^{\mu} \partial_{i} f^{\nu} .
$$

For $\left(M^{n}, g\right),\left(N^{n}, h\right)$ of bounded geometry up to order $k$ and $m \leq k$ we denote by $C^{\infty, m}(M, N)$ the set of all $f \in C^{\infty}(M, N)$ satisfying

$$
{ }^{b, m}|d f|:=\sum_{\mu=0}^{m-1} \sup _{x \in M}\left|\nabla^{\mu} d f\right|_{x}<\infty
$$

Assume $\left(M^{n}, g\right),\left(N^{n}, h\right)$ are open, complete, and of bounded geometry up to order $k, r \leq k, 1 \leq p<\infty, r>\frac{n}{p}+1$. Consider $C^{\infty, m}(M, N)$. According to the Sobolev embedding theorem, for $r>\frac{n}{p}+s$,

$$
\begin{gathered}
\Omega^{p, r}\left(f^{*} T N\right) \hookrightarrow^{b, s} \Omega\left(f^{*} T N\right), \\
{ }^{b, s}|Y| \leq D \cdot|Y|_{p, r},
\end{gathered}
$$

where $|Y|_{p, r}=\left(\int \sum_{i=0}^{r}\left|\nabla^{i} Y\right|^{p} d \mathrm{vol}\right)^{\frac{1}{p}}$. Set for $\delta>0, \delta \cdot D \leq \delta_{N}<r_{i n j}(N) / 2,1 \leq p<\infty$, $V_{\delta}=\left\{(f, g) \in C^{\infty, m}(M, N) \times C^{\infty, m}(M, N) \mid \exists Y \in \Omega_{r}^{p}\left(f^{*} T N\right)\right.$ such that $g=g_{Y}=$ $\exp Y$ and $\left.|Y|_{p, r}<\delta\right\}$. 
Proposition 2.27. $\mathfrak{B}=\left\{V_{\delta}\right\}_{0<\delta<r_{i n j}(N) / 2 D}$ is a basis for a metrizable uniform structure $\mathfrak{U}^{p, r}\left(C^{\infty, m}(M, N)\right)$.

$\mathfrak{U}^{p, r}\left(C^{\infty, m}(M, N)\right)$ is metrizable. Let ${ }^{m} \Omega^{p, r}(M, N)$ be the completion of $C^{\infty, m}(M, N)$. From now on we assume $r=m$ and deonte ${ }^{r} \Omega^{p, r}(M, N)=\Omega^{p, r}(M, N)$.

TheOrem 2.28. Let $\left(M^{n}, g\right),\left(N^{n}, h\right)$ be open, complete, of bounded geometry up to order $k, 1 \leq p<\infty, r \leq k, r>\frac{n}{p}+1$. Then each component of $\Omega^{p, r}(M, N)$ is a $C^{1+k-r}-$ Banach manifold, and for $p=2$ it is a Hilbert manifold.

Let $\left(M^{n}, g\right)$ be open, complete, oriented, of bounded geometry up to order $k$, $1 \leq p<\infty, r \leq k, r>\frac{n}{p}+1$. Set

$$
\mathcal{D}^{p, r}=\left\{f \in \Omega^{p, r}(M, M) \mid f \quad \text { bijective, } \quad \text { orientation } \quad \text { preserving, } \quad|\lambda|_{\min }(d f)>0 .\right\}
$$

THEOREM 2.29. $\mathcal{D}^{p, r}$ is open in $\Omega^{p, r}(M, M)$, in particular, each component is a $C^{1+k-r}$-Banach manifold.

This follows from immediately from the condition $|\lambda|_{\min }(d f)>0$ and the Sobolev embedding theorem. In particular for $f \in \mathcal{D}^{2, r}, T_{f} \mathcal{D}^{2, r}=T_{f} \Omega^{2, r}(M, M)$, but for $f \in \mathcal{D}^{2, r}, \exp _{f}: B_{\varepsilon}(0) \subset T_{f} \mathcal{D}^{2, r} \rightarrow \Omega^{2, r}(M, M)$, must not map into $\mathcal{D}^{2, r}$. This only holds for $\varepsilon$ "very small", depending on $|\lambda|_{\min }(d f)$.

We denote by $\operatorname{comp}^{2, r}(i d)=\mathcal{D}_{0}^{2, r} \subset \mathcal{D}^{2, r}$ or $\operatorname{comp}^{2, r}(i d) \subset \Omega^{2, r}(M, M)$ the components of the identity map, respectively. $f \in \operatorname{comp}(i d) \subset \mathcal{D}^{2, r}$ or $f \in \operatorname{comp}(i d) \subset$ $\Omega^{2, r}(M, M)$ if and only if $f$ is homotopic in $\mathcal{D}_{0}^{2, r}$ or $\Omega^{2, r}(M, M)$ to the identity map.

The metric $g$ enters into the definition of $\mathcal{D}_{0}^{2, r}$ and $\Omega^{2, r}(M, M)$. It is easy to show that they depend only on $c o m p^{2, r}(g)$,

$$
\begin{gathered}
\mathcal{D}_{0}^{2, r}(g)=\mathcal{D}_{0}^{2, r}(\operatorname{comp}(g)), \\
\Omega^{2, r}((M, g),(M, g))=\Omega^{2, r}((M, \operatorname{comp}(g)),(M, \operatorname{comp}(g))) .
\end{gathered}
$$

In particular, if we fix a metric $g_{0} \in \mathcal{M}\left(I, B_{k}\right)$ and consider a smooth curve $\left\{g_{t}\right\}_{0 \leq t \leq 1}$ connecting $g_{0}$ and $g_{1}$ in $\mathcal{M}\left(I, B_{k}\right)$, then

$$
\Omega^{2, r}\left(\left(M, g_{0}\right),\left(M, g_{0}\right)\right)=\Omega^{2, r}\left(\left(M, g_{0}\right),\left(M, g_{t}\right)\right) .
$$

This will be important in section 7 .

3. Harmonic maps as stationary points. Not very much is known about harmonic maps between open manifolds. The reason for this is that-in comparison with the compact case-the analytical difficulties grow considerably. Nevertheless, there are some substantial results, e.g. [3], [19], [27], [31].

We start with some generalities concerning energy, tension and harmonic maps between open manifolds.

Let $\left(M^{n}, g\right),\left(N^{n^{\prime}}, h\right)$ be open and of bounded geometry up to order $k, k \geq r>$ $\max \left\{n, n^{\prime}\right\}+2, p=1, f \in \Omega^{1, r}(M, N)$. Then $d f \in C^{1}\left(T^{*} M \otimes f^{*} T N\right)$. Let us introduce the energy density of $f$,

$$
e(f)=\frac{1}{2}|d f|_{T^{*} M \otimes f^{*} T N}^{2}
$$


If $\operatorname{vol}(M, g)=\infty\left(\right.$ as follows from $(I)$ and $\left.\left(B_{k}\right)\right)$ then, in general,

$$
E(f):=\int_{M} e(f) d \operatorname{vol}_{x}(g)=\infty .
$$

Let $X \in \Omega^{1, r}\left(f^{*} T N\right)$, where $f^{*} T N$ is endowed with induced metric and connection, and put $f_{t}=\exp t X$, i.e.

$$
f_{t}(x)=\exp _{f(x)}\left(t X_{f(x)}\right), \quad e\left(f_{t}\right)=\frac{1}{2}\left|d f_{t}\right|_{T^{*} M \otimes f_{t}^{*} T N}^{2} .
$$

Lemma 3.1. Let $f, X, f_{t}$ as above. Then the integral

$$
\begin{gathered}
\int_{M}\left[e\left(f_{t}\right)-e(f)\right] d \operatorname{vol}_{x}(g) \\
=\frac{1}{2} \int_{M}\left[\left(d f_{t}, d f_{t}\right)-(d f, d f)\right] d \operatorname{vol}_{x}(g)
\end{gathered}
$$

converges, i.e. has a finite value.

Proof. $d f_{t}-d f$ is not defined since for a tangent vector $Z \in T_{x} M, d f_{t}(Z)$ and $d f(Z)$ lie in different tangent spaces. But if we translate $d f_{t}$ along $\exp \left(-s P_{t} X\right)$ from $f_{t}(x)$ to $f(x)$, thus getting a map $\widetilde{d f_{t}}$, then $\widetilde{d f}_{t}-d f$ is well defined. We obtain

$$
\left(d f_{t}, d f_{t}\right)-(d f, d f)=\left(\widetilde{d f_{t}}, \widetilde{d f_{t}}\right)-(d f, d f)=\left(\widetilde{d f_{t}}+d f, \widetilde{d f_{t}}-d f\right) .
$$

But $\left|\widetilde{d f_{t}}\right|,|d f|$ are bounded and $\left|\widetilde{d f_{t}}-d f\right|_{x} \leq C \cdot t|X|$, where $C$ depends on curvature bounds. Hence the integral (3.2) converges. $\square$

Lemma 3.2. Let $f_{1} \in \operatorname{comp}^{1, r}\left(f_{0}\right)$. Then

$$
\int_{M}\left[e\left(f_{1}\right)-e\left(f_{0}\right)\right] d \operatorname{vol}_{x}(g)
$$

converges, i.e. has a finite value.

Proof. By assumption, $f_{1}=\exp X_{u} \circ \exp X_{u-1} \cdots \circ \exp X_{1}, X_{1} \in \Omega^{1, r}\left(f_{0}^{*} T N\right)$, $X_{i} \in \Omega^{1, r}\left(\left(\exp X_{i-1} \cdots \circ \exp X_{1}\right)^{*} T N\right)$. Then we write

$$
\begin{aligned}
& e\left(f_{1}\right)-e\left(f_{0}\right) \\
& =e\left(\exp X_{u} \circ \exp X_{u-1} \cdots \circ \exp X_{1}\right)-e\left(\exp X_{u-1} \cdots \circ \exp X_{1}\right) \\
& +e\left(\exp X_{u-1} \cdots \circ \exp X_{1}\right)-e\left(\exp X_{u-2} \cdots \circ \exp X_{1}\right) \\
& +\cdots \\
& +e\left(\exp X_{1}\right)-e\left(f_{0}\right)
\end{aligned}
$$

Each of the terms (3.4)-(3.6) is of the kind (3.1) and we can apply Lemma 3.1. $\square$

Define for $f_{0} \in \Omega^{1, r}(M, N)$ the relative energy functional w.r.t. $f_{0}$,

$$
E_{f_{0}}: \operatorname{comp}^{1, r}\left(f_{0}\right) \rightarrow \mathbb{R}
$$


by

$$
E_{f_{0}}(f)=\int_{M}\left[e(f)-e\left(f_{0}\right)\right] d \operatorname{vol}_{x}(g) .
$$

According to Lemma $3.2, E_{f_{0}}(\cdot)$ is well defined. Let us further define for $f \in$ $\Omega^{1, r}(M, N)$ the tension field $\tau(f) \in^{b, 0} \Omega\left(f^{*} T N\right)=T_{f}^{b, 0} \Omega(M, N)$ by

$$
\tau(f):=\operatorname{tr}_{g} \nabla^{h} d f=\left\{\tau^{\alpha}(f)\right\}_{\alpha},
$$

$\tau^{\alpha}(f)=\left(g^{i j}\left(\nabla^{h} d f\right)_{i j}\right)^{\alpha}, g=\left(g_{i j}\right), h=\left(h_{\alpha \beta}\right), i, j=1,2, \cdots, n, \quad \alpha, \beta=1,2, \cdots, n^{\prime}$.

Lemma 3.3. A map $f \in \operatorname{comp}^{1, r}\left(f_{0}\right)$ is a stationary point of $E_{f_{0}}(\cdot)$ if and only if

$$
\tau(f)=\operatorname{tr}_{g} \nabla^{h} d f=0 .
$$

Proof. We note that our choice of $r$ implies the applicability of Stokes' theorem. We have to calculate for $f_{t X}=\exp t X, X \in \Omega^{1, r}\left(f^{*} T N\right)$,

$$
\begin{aligned}
\left.\frac{d}{d t} E_{f_{0}}\left(f_{t X}\right)\right|_{t=0} & =\left.\frac{1}{2} \int_{M} \frac{d}{d t}\left[\left(d f_{t X}, d f_{t X}\right)-\left(d f_{0}, d f_{0}\right)\right]\right|_{t=0} d \operatorname{vol}_{x}(g) \\
& =\left.\int_{M}\left(\nabla_{\frac{\partial}{\partial t}} d f_{t X}, d f\right)\right|_{t=0} d \operatorname{vol}_{x}(g) \\
& =\int_{M}(d f, \nabla X) d \operatorname{vol}_{x}(g) \\
& =\int_{M}\left(\nabla^{*} d f, X\right) d \operatorname{vol}_{x}(g) \\
& =-\int_{M}\left(t r_{g} \nabla d f, X\right) d \operatorname{vol}_{x}(g),
\end{aligned}
$$

hence the Euler-Lagrange equations for $E_{f_{0}}(\cdot)$ are

$$
\tau(f)=\operatorname{tr}_{g} \nabla^{h} d f=0 .
$$

Lemma 3.4. Let $f_{1} \in \operatorname{comp}^{1, r}\left(f_{0}\right)$. Then $f$ is a stationary point of $E_{f_{0}}(\cdot)$ if and only if it is a stationary point of $E_{f_{1}}(\cdot)$.

Now we define $f \in \Omega^{1, r}(M, N)$ to be harmonic if it is a stationary point of some $E_{f_{0}}(\cdot), f_{0} \in \operatorname{comp}^{1, r}(f)$ fixed. As we have seen, this property does not depend on the choice of $f_{0}$.

We summarize in

Proposition 3.5. $f \in \Omega^{1, r}(M, N)$ is harmonic if and only if

$$
\tau(f)=\operatorname{tr}_{g} \nabla^{h} d f=0 .
$$


We now turn to harmonic maps within the Teichmüller space. Suppose $M^{2}=N^{2}$, $M^{2}$ an open Riemannian surface with $g_{0} \in \mathcal{M}\left(I, B_{\infty}\right), K_{g_{0}} \equiv-1\left(K_{g_{0}}=\right.$ the scalar curvature), $r_{i n j}\left(g_{0}\right)>0$, inf $\sigma_{e}\left(\Delta_{0}\left(g_{0}\right)\right)>0$. We extend our considerations above slightly by working with $p=2$. But for vector bundles, $\Omega^{1, r} \hookrightarrow \Omega^{2, \frac{r}{2}}$ if $r>n$ : $r-\frac{n}{1}>\frac{r}{2}-\frac{n}{2}, r>\frac{n}{2}, p=2>1=q$. This implies $\Omega^{1, r}(M, N) \hookrightarrow \Omega^{2, \frac{r}{2}}(M, N)$ etc. We write in the sequel $r$ for $\frac{r}{2}$ and assume $r>\frac{n}{2}+2$, i.e. in our case $r>3$. Denote as in section 2 by comp $p^{2, r}\left(g_{0}\right)_{-1}$ the submanifold consisting of all metrics of scalar curvature -1. Let $\left\{g_{t}\right\}_{0 \leq t \leq 1}$ be a $C^{r}$ - curve in $c o m p^{2, r}\left(g_{0}\right)_{-1}$. Then $i d:\left(M^{2}, g_{0}\right) \rightarrow\left(M^{2}, g_{0}\right)$ is harmonic. We ask for a harmonic map $f_{1} \in \operatorname{comp}^{2, r}(i d) \subset \Omega^{2, r}\left(\left(M, g_{0}\right),\left(M, g_{1}\right)\right)$ between $\left(M, g_{0}\right)$ and $\left.\left(M, g_{1}\right)\right)$.

Consider the map

$$
\begin{aligned}
& {[0,1] \times \operatorname{comp}^{2, r}(i d) \underset{\tilde{F}}{\longrightarrow} T \operatorname{comp} p^{2, r-2}(i d),} \\
& \tilde{F}(t, f):=t r_{g_{0}} \nabla^{g_{t}} d f \in T c o m p^{2, r-2}(i d) .
\end{aligned}
$$

First we must show that this map is well defined, i.e.

$$
\tau_{t}(f)=t r_{g_{0}} \nabla^{g_{t}} d f \in \Omega^{2, r-2}\left(f^{*} T M^{2}, f^{*} g_{t}\right) \cong \Omega^{2, r-2}\left(T M^{2}, g_{0}\right) .
$$

At this point we should remove the $t$-ambiguity in our Sobolev spaces. According to section 2 ,

$$
\Omega^{2, j}\left(T M, g_{0}\right)=\Omega^{2, j}\left(T M, g_{t}\right), \quad 0 \leq j \leq r-1, \quad 0 \leq t \leq 1,
$$

as equivalence of Sobolev spaces and, since $\left\{g_{t}\right\}_{0 \leq t \leq 1}$ is a compact curve, there exists constants independent of $t$ which describe the equivalence (3.10). Moreover, for any $C^{r}$ - curve $\left\{f_{t}\right\}_{0 \leq t \leq 1}$ in $\operatorname{comp}^{2, r}(i d) \subset \Omega^{2, r}\left(\left(M, g_{0}\right),\left(M, g_{0}\right)\right) \equiv \Omega^{2, r}\left(\left(M, g_{0}\right),\left(M, g_{t}\right)\right)$,

$$
\Omega^{2, j}\left(T M, g_{0}\right) \cong \Omega^{2, j}\left(f_{t}^{*} T M, f_{t}^{*} g_{\tau}\right), \quad 0 \leq j \leq r-1, \quad 0 \leq t \leq 1, \quad 0 \leq \tau \leq 1,
$$

with equivalence constants independent of $t, \tau$ (but depending on $\left\{g_{\tau}\right\}_{0 \leq \tau \leq 1}$, $\left.\left\{f_{t}\right\}_{0 \leq t \leq 1}\right)$. Finally, we use the fact that under our assumptions the Sobolev spaces based on the covariant derivative $\nabla$ and on the (Bochner) Laplacian $\Delta$ are equivalent, i.e.

$$
\begin{gathered}
\Omega^{2, j}\left(T M, \Delta^{g_{0}}, g_{0}\right) \cong \Omega^{2, j}\left(T M, \nabla^{g_{0}}, g_{0}\right) \\
\Omega^{2, j}\left(T M, \nabla^{g_{t}}, g_{t}\right) \cong \Omega^{2, j}\left(T M, \Delta^{g_{0}}, g_{t}\right), \quad 0 \leq j \leq r-1, \quad 0 \leq t \leq 1
\end{gathered}
$$

as equivalent Sobolev spaces with constants independent of $t$ (cf. [12]. (3.12) holds correspondingly for the induced bundles and metrics by a curve $\left\{f_{t}\right\}_{0 \leq t \leq 1}$ in $\operatorname{comp}^{2, r}(i d)$. We want to prove $\tau_{t}(f)=t r_{g_{0}} \nabla_{g_{t}} d f \in \Omega^{2, r-2}\left(f^{*} T M\right)$ and start with the case $f=\exp X$. At this point there arises a further question. With respect to which $g$ shall we take the exponential map, $f=\exp ^{g} X$ ? Let $\delta<\inf _{t} r_{i n j}\left(g_{t}\right)$. Then $f=\exp ^{g_{t}} X\left(g_{t}\right)$ if and only if $f=\exp ^{g_{0}} X\left(g_{0}\right)$ if we restrict to $X^{\prime} s$ with length $\leq \delta$, i.e. given $f$ and $t$, there exists a unique $X=X\left(g_{t}\right)$ such that $f=\exp ^{g_{t}} X\left(g_{t}\right)$. Hence, restricting once and for all to such $X^{\prime} s$, the choice of $g$ to represent $f$ as $f=\exp ^{g} X$ is irrelevent and we fix $g \equiv g_{0}$ and simply write $f=\exp X=\exp ^{g_{0}} X$. Moreover, we denote in the sequel $\nabla \equiv \nabla^{g_{0}}$. 
Proposition 3.6. Let $\left(M^{2}, g_{t}\right), r$ be as above and $f \in \operatorname{comp}^{2, r}(i d) \subset$ $\Omega^{2, r}\left(\left(M, g_{0}\right),\left(M, g_{t}\right)\right)$. Then

$$
\tau_{t}(f)=t r_{g_{0}}\left(\nabla^{g_{t}} d f\right) \in \Omega^{2, r-2}\left(f^{*} T M\right) \equiv T_{f} c o m p^{2, r}(i d) .
$$

Proof. We start with $f=\exp X \equiv \exp ^{g_{0}} X\left(g_{0}\right), X \in \Omega_{r}^{2}(T M)$. Then, according to chapter III, section 5 of [12], for $1 \leq i \leq r-1$

$$
\left|\nabla^{i} d \exp X\right|_{x} \leq p_{i}\left(|X|_{x},|\nabla X|_{x}, \cdots,\left|\nabla^{i} X\right|_{x}\right) \in L_{2},
$$

where $p_{i}$ is a universal polynomial in the indicated variables without constant term. (3.14) implies

$$
\begin{aligned}
& \left|\operatorname{tr}_{g_{0}} \nabla^{g_{0}} d \exp X\right| \in L_{2}, \\
& \left|\operatorname{tr}_{g_{0}} \nabla^{g_{t}} d \exp X\right| \in L_{2}
\end{aligned}
$$

and finally

$$
\left|\nabla_{g_{0}}^{j} t r_{g_{0}} \nabla^{g_{t}} d \exp X\right| \in L_{2}, \quad 0 \leq j \leq r-2 .
$$

Let $X \in \Omega^{2, r}(T M)$, then there exists a sequence $\left(X_{\nu}\right)_{\nu}$ in $\Omega_{r}^{2}(T M)$ converging to $X$ w.r.t. $|\cdot|_{r}$. For the $X_{\nu}$ (3.14) makes sense since the corresponding pointwise norms are defined. We have, according to the embedding theorem, $X_{\nu} \rightarrow X$ in $C^{2}$, $t r_{g_{0}} \nabla d \exp X_{\nu} \rightarrow t r_{g_{0}} \nabla d \exp X$ in $C^{0}$, and $\left(\nabla^{j} t r_{g_{0}} \nabla d \exp X_{\nu}\right)_{\nu}$ is a Cauchy sequence in $L_{2}$ which converges to (the distribution) $\nabla^{j} t_{g_{0}} \nabla d \exp X$, which now is in $L_{2}$ too, $0 \leq j \leq r-2$. Then, according to the equivalence of Sobolev spaces above,

$$
\left|\nabla_{g_{0}}^{j} t r_{g_{0}} \nabla^{g_{t}} d \exp X\right| \in L_{2}, \quad 0 \leq j \leq r-2 .
$$

Suppose

$$
\left|\nabla^{i} d f\right|_{x} \leq p_{i, u-1}\left(\left|X_{1}\right|, \cdots,\left|\nabla^{i} X_{1}\right|, \cdots\left|X_{u-1}\right|, \cdots,\left|\nabla^{i} X_{u-1}\right|\right) \in L_{2}
$$

for all $f=\exp X_{u-1} \circ \cdots \circ \exp X_{1}, X_{j} \in \Omega_{r}^{2}\left(\left(\exp X_{j-1} \cdots \circ \exp X_{1}\right)^{*} T M\right)$ and let now $f=$ $\exp X_{u} \circ \exp X_{u-1} \cdots \circ \exp X_{1}, \sup _{x, j}\left|X_{j}\right|_{x}<\inf _{t} r_{i n j}\left(g_{t}\right)$. We recall: If $E_{0}, E_{1}, \cdots, E_{u}$ are vector bundles with connections $\nabla_{0}, \cdots, \nabla_{u}$, respectively, which induce connections $\nabla=\nabla_{j-1, j}$ in $\operatorname{Hom}\left(E_{j-1}, E_{j}\right)$ and $\Phi: \Gamma\left(E_{0}\right) \rightarrow \Gamma\left(E_{u}\right)$ can be factorized as $\Phi:$ $\Gamma\left(E_{0}\right) \stackrel{\Phi_{1}}{\longrightarrow} \Gamma\left(E_{1}\right) \rightarrow \cdots \stackrel{\Phi_{u}}{\longrightarrow} \Gamma\left(E_{u}\right)$ then for $X \in \Gamma$

$$
\begin{aligned}
\nabla(\Phi(X))= & \nabla\left(\left(\Phi_{u} \circ \cdots \circ \Phi_{1}\right)(X)\right) \\
= & \left(\nabla \Phi_{u}\right)\left(\Phi_{u-1} \circ \cdots \circ \Phi_{1}\right)(X)+\Phi_{u} \circ\left(\nabla \Phi_{u-1}\right)\left(\Phi_{u-2} \circ \cdots \circ \Phi_{1}\right)(X) \\
& +\cdots+\left(\Phi_{u} \circ \cdots \circ \Phi_{1}\right)(\nabla X) .
\end{aligned}
$$

We apply this to $E_{0}=T M, \Phi_{j}=d \exp X_{j}$. Hence

$$
\begin{aligned}
\nabla\left(d \exp X_{u} \circ \cdots \circ d \exp X_{1}\right)= & \nabla d\left(\exp X_{u} \circ \cdots \circ \exp X_{1}\right) \\
= & {\left[\nabla\left(d \exp X_{u}\right)\right] \circ d\left(\exp X_{u-1} \circ \cdots \circ \exp X_{1}\right) } \\
& +d \exp X_{u} \circ \nabla d\left(\exp X_{u-1} \circ \cdots \circ \exp X_{1}\right)
\end{aligned}
$$


Here $\left|\nabla d\left(\exp X_{u}\right)\right| \leq p_{1}\left(\left|X_{u}\right|,\left|\nabla X_{u}\right|\right)=p_{1,1}$ and $d\left(\exp X_{u-1} \circ \cdots \circ \exp X_{1}\right)$ is bounded by $p_{0, u-1}\left(\left|X_{1}\right|, \cdots,\left|X_{u-1}\right|\right)$ since we assumed $\left(B_{1}\right)$ and $r>3, d \exp X_{u}$ is bounded by $p_{0,1}$ and $\left|\nabla d\left(\exp X_{u-1} \circ \cdots \circ \exp X_{1}\right)\right| \leq p_{1, u-1} \in L_{2}$ by induction assumption. We infer

$$
\left|\nabla d\left(\exp X_{u} \circ \cdots \circ \exp X_{1}\right)\right| \leq p_{1,1} \cdot p_{0, u-1}+p_{0,1} \cdot p_{1, u-1}=p_{1, u} .
$$

We demonstrate the general rule, performing the estimates still for $\nabla^{2}$. We apply $\nabla$ to (3.21) and obtain

$$
\begin{aligned}
& {\left[\nabla^{2}\left(d \exp X_{u}\right)\right] \circ d\left(\exp X_{u-1} \circ \cdots \circ \exp X_{1}\right) } \\
+ & {\left[\nabla\left(d \exp X_{u}\right)\right] \circ\left[\nabla d\left(\exp X_{u-1} \circ \cdots \circ \exp X_{1}\right)\right] } \\
+ & {\left[\nabla\left(d \exp X_{u}\right)\right] \circ\left[\nabla d\left(\exp X_{u-1} \circ \cdots \circ \exp X_{1}\right)\right] } \\
+ & {\left[d \exp X_{u}\right] \circ\left[\nabla^{2} d\left(\exp X_{u-1} \circ \cdots \circ \exp X_{1}\right)\right], }
\end{aligned}
$$

$\left|\nabla^{2}\left(d \exp X_{u}\right)\right| \leq p_{2}\left(\left|X_{u}\right|,\left|\nabla X_{u}\right|,\left|\nabla^{2} X_{u}\right|\right)=p_{2,1} \in L_{2},\left|d\left(\exp X_{u-1} \circ \cdots \circ \exp X_{1}\right)\right|$ is bounded by $p_{0, u-1},\left|\nabla d \exp X_{u}\right| \leq p_{1,1}\left(\left|X_{u}\right|,\left|\nabla X_{u}\right|\right) \in L_{2}, \mid \nabla d\left(\exp X_{u-1} \circ \cdots \circ\right.$ $\left.\exp X_{1}\right)\left|\leq p_{1, u-1} \in L_{2},\right| \nabla^{2} d\left(\exp X_{u-1} \circ \cdots \circ \exp X_{1}\right) \mid \leq p_{1, u-1} \in L_{2}$, hence

$$
\left|\nabla^{2} d\left(\exp X_{u-1} \circ \cdots \circ \exp X_{1}\right)\right| \leq p_{2,1} \cdot p_{0, u-1}+2 p_{1,1} \cdot p_{1, u-1}+p_{0,1} \cdot p_{2, u-1} .
$$

The polynomials $p_{2,1}, p_{2, u-1}$ are in $L_{2}, p_{0, j}$ is always bounded, $p_{2,1} \cdot p_{0, u-1}, p_{0,1}$. $p_{2, u-1}$ are in $L_{2}$. The open question is $p_{1,1} \cdot p_{1, u-1} \in L_{2}$ ? Fortunately, the product $p_{1,1} \cdot p_{1, u-1}$ in (3.27) is indeed in $L_{2}$. This comes from the special structure of the polynomials which we exhibited in chapter III, section 5 and 6 of [12]. We repeat this briefly. Assuming the assumptions of the module structure theorem-which are satisfied in our case-one gets $X, Y(2, r)$-Sobolev implies $X \otimes Y$ is also $(2, r)$-Sobolev. The $p_{i j}$ arise during the estimate of the i-th derivative of $j$ factors (or compositions) applying the Leibniz rule. The module structure theorem then just says that the estimated summands are in $L_{2}$. This yields $p_{1,1} \cdot p_{1, u-1} \in L_{2}$. Having these arguments in mind, it is a matter of induction and simple combinatorics that, assuming $\mid \nabla^{j} d \exp X_{u} \circ \cdots \circ$ $\left.\exp X_{1}\right) \mid \leq p_{j, u}$, finally

$$
\left|\nabla^{j+1} d\left(\exp X_{u} \circ \cdots \circ \exp X_{1}\right)\right| \leq p_{j+1, u}, \quad j+1 \leq r-1,
$$

where $p_{j+1, u}$ with the structure coming from (3.19). By a simple completion (diagonal) argument quite parallel to that after (3.17) we can extend (3.28) to the case $X_{j} \in \Omega^{2, r}\left(\left(\exp X_{j-1} \circ \cdots \circ \exp X_{1}\right)^{*} T M\right)$, i.e. to the case $f \in c o m p^{2, r}(i d)$ and infer from (3.28), (3.11) that

$$
\begin{aligned}
& \left|\operatorname{tr}_{g_{0}} \nabla d f\right| \in L_{2}, \\
& \left|\operatorname{tr}_{g_{0}} \nabla^{g_{t}} d f\right| \in L_{2}, \\
& \left|\nabla^{j} \operatorname{tr}_{g_{0}} \nabla^{g_{t}} d f\right| \in L_{2}, \quad j \leq r-2, \\
& \text { i.e. } \quad \tau_{t}(f)=\operatorname{tr}_{g_{0}} \nabla^{g_{t}} d f \in \Omega^{2, r-2}\left(f^{*} T M\right) .
\end{aligned}
$$


4. Gradient estimates for harmonic maps on open manifolds. We assume that for a curve $\left\{g_{t}\right\}:=\gamma$ of metrics, we have a family of harmonic diffeomorphisms $f_{t}:\left(M^{2}, g_{0}\right) \rightarrow\left(M^{2}, g_{t}\right)$ for $t \in[0, T]$. This implies

$$
\tau_{t}\left(f_{t}\right) \equiv \operatorname{tr}_{g_{0}}\left(\nabla_{g_{t}} d f_{t}\right)=0 .
$$

For $f_{0}=i d:\left(M, g_{0}\right) \rightarrow\left(M, g_{t}\right)$, we have

$$
\tau_{t}\left(f_{0}\right)=B(i d)\left(i d_{*} e_{i}, i d_{*} e_{i}\right)=\nabla_{e_{i}}^{g_{t}} e_{i}-\nabla_{e_{i}}^{g_{0}} e_{i}=\left(\Gamma_{t, i i}^{k}-\Gamma_{0, i i}^{k}\right) \partial_{y^{k}},
$$

where $B(i d)(\cdot, \cdot)$ stands for the second fundamental form of the map $i d:\left(M, g_{0}\right) \rightarrow$ $\left(M, g_{t}\right)$, and $\left\{\partial_{y^{k}}\right\}$ is a natural frame corresponding to some local coordinates $\left\{y^{k}\right\}$ on $M$. Using the assumption on the metrics $\left\{g_{t}\right\}$, we have

$$
\left|\tau_{t}(i d)\right|_{g_{t}}=\left|\left(\Gamma_{t, i i}^{k}-\Gamma_{0, i i}^{k}\right) \partial_{y^{k}}\right|_{g_{t}} \in L^{2}(M) .
$$

As in [23], we have the distance function $\rho_{t}(\cdot, \cdot)$ with respect to the metric $g_{t}$ on $M$. From [23] and [3], we have

$$
\Delta \rho_{t}\left(f_{t}, i d\right) \geq-\left(\left|\tau_{t}\left(f_{t}\right)\right|_{g_{t}}+\left|\tau_{t}(i d)\right|_{g_{t}}\right),
$$

consequently,

$$
\Delta \rho_{t}\left(f_{t}, i d\right) \geq-\left|\tau_{t}(i d)\right|_{g_{t}}
$$

and note that the RHS is in $L^{2}(M)$, furthermore, the $L^{2}$ norm of $\left|\tau_{t}(i d)\right|_{g_{t}}$ is bounded above by a constant $C=C(\gamma)$ independent of $t$. For simplicity of notation, we denote the lowest spectral value $\inf \sigma\left(\Delta_{0}\left(g_{0}\right)\right)>0$ of $\left(M, g_{0}\right)$ by $\lambda\left(M, g_{0}\right)$, or even simply $\lambda(M)$.

LEMMA 4.1. We have

$$
\int_{M} \rho_{t}^{2}\left(f_{t}, i d\right) d v_{0} \leq C_{0}<+\infty
$$

where $C_{0}=C_{0}\left(\gamma, \lambda\left(M, g_{0}\right)\right)>0$ is a constant independent of $t$.

We proceed to consider the gradient estimates for $f_{t}$ and will prove Lemma 4.1 later. Since $\lambda\left(M, g_{0}\right)>0, R i c_{M} \geq-1$, and $\inf _{x \in M} \operatorname{Vol}\left(B_{x}(1) \equiv a>0\right.$, from (4.2) and using the Moser's iteration, we have (c.f. Proposition 2.1 and (3.5) in [3]):

$$
\rho_{t}\left(f_{t}, i d\right)(x) \leq C_{1}\left[\left\|\rho_{t}\left(f_{t}, i d\right)\right\|_{L^{2}\left(B_{x}(2)\right)}+\operatorname{Vol}\left(B_{x}(2)\right)^{\frac{1}{2}}\left\|\tau_{t}(i d)\right\|_{g_{t}, L^{2}\left(B_{x}(2)\right)}\right]
$$

for any $x \in M$, where $C_{1}>0$ is a constant independent of $t$. Since

$$
\left\|\rho_{t}\left(f_{t}, i d\right)\right\|_{L^{2}(M)} \leq C_{2}, \quad\left\|\tau_{t}(i d)\right\|_{g_{t}, L^{2}(M)} \leq C_{2}
$$

for some constant $C_{2}>0$ independent of $t$, we conclude that

$$
\rho_{t}\left(f_{t}, i d\right)(x) \rightarrow 0 \quad(x \rightarrow \infty)
$$

uniformly in $t$, hence

$$
\rho_{t}\left(f_{t}, i d\right)(x) \leq C_{3}, \quad \forall t, \forall x \in M,
$$


where $C_{3}>0$ is a constant independent of $t$.

REMARK 4.2. From (4.5) we have

$$
d_{g_{t}}\left(f_{t}(x), x\right) \leq C_{3}, \quad \forall t, \forall x \in M,
$$

so, for any $x_{0} \in M$ and a geodesic ball in $\left(M, g_{0}\right)$ of radius $r$ centered at $x_{0}$, we have

$$
\begin{aligned}
d_{g_{t}}\left(f_{t}(x), f_{t}\left(x_{0}\right)\right) & \leq d_{g_{t}}\left(f_{t}(x), x\right)+d_{g_{t}}\left(x, x_{0}\right)+d_{g_{t}}\left(f_{t}\left(x_{0}\right), x_{0}\right) \\
& \leq 2 C_{3}+C_{4} r:=R(r),
\end{aligned}
$$

where in the last step we have used the fact that

$$
C_{4}^{-1} g_{0} \leq g_{t} \leq C_{4} g_{0}
$$

for some constant $C_{4}>0$ independent of $t$. Therefore, for the map $f_{t}:\left(M, g_{0}\right) \rightarrow$ $\left(M, g_{t}\right)$,

$$
f_{t}\left(B_{x_{0}}(r)\right) \subset B_{f_{t}\left(x_{0}\right)}(R(r)) .
$$

Denote by $\widetilde{M}$ the universal covering space of $M$, and denote the covering projection by $\pi$. For any $x \in M$, choose $\tilde{x} \in \pi^{-1}(x)$. Let $\delta>0$ be a constant smaller than the infimum of the injective radius of points in $\left(M, g_{0}\right)$ which is positive since $\left(M, g_{0}\right)$ satisfies (I). Then from (4.5), for any $\tilde{x}^{\prime} \in \tilde{B}_{\tilde{x}}(\delta)$,

$$
\begin{aligned}
d_{\pi^{*} g_{t}}\left(\tilde{f}_{t}\left(\tilde{x}^{\prime}\right), \tilde{f}_{t}(\tilde{x})\right) & \leq d_{\pi^{*} g_{t}}\left(\tilde{f}_{t}\left(\tilde{x}^{\prime}\right), \tilde{x}^{\prime}\right)+d_{\pi^{*} g_{t}}\left(\tilde{x}^{\prime}, \tilde{x}\right)+d_{\pi^{*} g_{t}}\left(\tilde{f}_{t}(\tilde{x}), \tilde{x}\right) \\
& \leq \rho_{t}\left(f_{t}, i d\right)\left(x^{\prime}\right)+C_{4} \delta+\rho_{t}\left(f_{t}, i d\right)(x) \\
& \leq 2 C_{3}+C_{4} \delta \equiv R_{0},
\end{aligned}
$$

namely, the harmonic map $\tilde{f}_{t}:\left(\tilde{M}, \pi^{*} g_{0}\right) \rightarrow\left(\tilde{M}, \pi^{*} g_{t}\right)$ satisfies:

$$
\tilde{f}_{t}\left(\tilde{B}_{\tilde{x}}(\delta)\right) \subset \tilde{B}_{\tilde{f}_{t}(\tilde{x})}\left(R_{0}\right)
$$

for some constant $R_{0}\left(\gamma, \lambda\left(M, g_{0}\right)\right)>0$ independent of $t$. Now we need the following (c.f. $[2])$

Lemma 4.3. Let $M^{m}$ and $N$ be complete Riemannian manifolds, $N$ is simplyconnected with nonpositive sectional curvature, $x_{0} \in M$. If $f: M \rightarrow N$ is a harmonic map satisfying $f\left(B_{x_{0}}(2)\right) \subset B_{y_{0}}(R) \subset N$, then

$$
\sup _{B_{x_{0}}(1)}|d f| \leq C(m, R, K)
$$

where $K \geq 0$, and Ric $_{M} \geq-K$ on $B_{x_{0}}(2)$.

Using the above estimate for $\tilde{f}_{t}: \tilde{B}_{\tilde{x}}(\delta) \rightarrow \tilde{B}_{\tilde{f}_{t}(\tilde{x})}\left(R_{0}\right)$, we have

$$
\left|d f_{t}\right|_{g_{t}}=\left|d \tilde{f}_{t}\right|_{\pi^{*} g_{t}}(\tilde{x}) \leq C_{5}
$$

for some constant $C_{5}>0$ independent of $t$. From the standard elliptic estimate for the equation

$$
\tau_{t}\left(f_{t}\right)=0
$$


we can conclude that

$$
\left|\nabla^{j} f_{t}\right|_{g_{t}}(x) \leq C(j, \gamma), \quad \forall x \in M, \quad j=1,2, \cdots, r+1,
$$

where $C(j, \gamma)>0$ is a constant independent of $t$. To see (4.10), we choose local coordinates $\left\{x^{\alpha}\right\},\left\{y^{i}\right\}$ around any point $x \in M$ and $f_{t}(x)$ respectively, and write (4.9) locally as

$$
\Delta f_{t}^{i}=-\Gamma_{t, j k}^{i} \frac{\partial f_{t}^{j}}{\partial x^{\alpha}} \frac{\partial f_{t}^{k}}{\partial x^{\beta}} g_{0}^{\alpha \beta}
$$

namely,

$$
\frac{\partial}{\partial x^{\alpha}}\left(\sqrt{g_{0}} g_{0}^{\alpha \beta} \frac{\partial f_{t}^{i}}{\partial x^{\beta}}\right)=-\sqrt{g_{0}} \Gamma_{t, j k}^{i} \frac{\partial f_{t}^{j}}{\partial x^{\alpha}} \frac{\partial f_{t}^{k}}{\partial x^{\beta}} g_{0}^{\alpha \beta}:=F_{t}^{i},
$$

where $g_{0}:=\operatorname{det}\left(g_{0, \alpha \beta}\right)$. It follows from (4.8) and $\Gamma_{t, j k}^{i} \in C^{r-1}(M)$ that $F_{t}^{i} \in L^{\infty}$, by Schauder estimates for the elliptic equation (4.12) (see e.g. Theorem 2.2.1 in [15] or [14]), we have

$$
\left\|f_{t}^{i}\right\|_{C^{1, \alpha}\left(B_{x}\left(\frac{1}{2}\right)\right)} \leq C(\gamma),
$$

using this in Schauder estimates for the elliptic equation (4.12) again, we obtain

$$
\left\|f_{t}^{i}\right\|_{C^{2, \alpha}\left(B_{x}\left(\frac{1}{2}\right)\right)} \leq C(\gamma),
$$

by using the bootstrap method like this, we finally obtain (4.10). Now we give the

Proof of Lemma 4.1. Since $f_{t} \in \operatorname{comp}(\mathrm{id})$, by definition (c.f. [12]), there exist vector fields $X_{1}^{t}, X_{2}^{t}, \cdots, X_{n_{t}}^{t}$ such that

$$
f_{t}=\exp X_{n_{t}}^{t} \circ \cdots \circ \exp X_{1}^{t}
$$

and $X_{j}^{t} \in \Omega^{2, r}\left(\left(\exp X_{j-1}^{t} \circ \cdots \circ \exp X_{1}^{t}\right)^{*} T M\right), j=1,2, \cdots, n_{t}$. The homotopic maps $f_{t}, i d:\left(M, g_{0}\right) \rightarrow\left(M, g_{t}\right)$ can be lifted to homotopic maps $\tilde{f}_{t}, i d:\left(\tilde{M}, \pi^{*} g_{0}\right) \rightarrow$ $\left(\tilde{M}, \pi^{*} g_{t}\right)$ respectively between covering spaces, and

$$
\tilde{f}_{t}=\exp \tilde{X}_{n_{t}}^{t} \circ \cdots \circ \exp \tilde{X}_{1}^{t}
$$

with $X_{j}^{t}=d \pi\left(\tilde{X}_{j}^{t}\right), \quad j=1,2, \cdots, n_{t}$. Note that since the covering projection $\pi$ is a local isometry, we have $\left|X_{j}^{t}\right|_{g_{t}}=\left|\tilde{X}_{j}^{t}\right|_{\pi^{*} g_{t}}, j=1,2, \cdots, n_{t}$. The distance between $\tilde{f}_{t}(\tilde{x})$ and $i d(\tilde{x})=\tilde{x}$ in $\left(\tilde{M}, \pi^{*} g_{t}\right)$ is

$$
\begin{aligned}
d_{\pi^{*} g_{t}}\left(\tilde{f}_{t}(\tilde{x}), \tilde{x}\right) & \leq d_{\pi^{*} g_{t}}\left(\tilde{x}, \exp \tilde{X}_{1}^{t}(\tilde{x})\right)+\cdots+d_{\pi^{*} g_{t}}\left(\exp \tilde{X}_{n_{t}-1}^{t} \circ \cdots \circ \exp \tilde{X}_{1}^{t}(\tilde{x}), \tilde{f}_{t}(\tilde{x})\right) \\
& =\left|\tilde{X}_{1}^{t}\right|_{\pi^{*} g_{t}}(\tilde{x})+\left|\tilde{X}_{2}^{t}\right|_{\pi^{*} g_{t}}(\tilde{x})+\cdot+\left|\tilde{X}_{n_{t}}^{t}\right|_{\pi^{*} g_{t}}(\tilde{x}) \\
& =\left|X_{1}^{t}\right|_{g_{t}}(x)+\left|X_{2}^{t}\right|_{g_{t}}(x)+\cdots+\left|X_{n_{t}}^{t}\right|_{g_{t}}(x),
\end{aligned}
$$

by the definition of $\rho_{t}$ we have

$$
\rho_{t}\left(f_{t}(x), x\right) \leq \sum_{i=1}^{n_{t}}\left|X_{i}^{t}\right|_{g_{t}}(x) .
$$


From this we conclude that

$$
\begin{aligned}
\int_{M} \rho_{t}^{2}\left(f_{t}, i d\right) d v_{0} & \leq \int_{M}\left(\sum_{i=1}^{n_{t}}\left|X_{i}^{t}\right|_{g_{t}}\right)^{2} d v_{0} \\
& \leq n_{t} \sum_{i=1}^{n_{t}} \int_{M}\left|X_{i}^{t}\right|_{g_{t}}^{2} d v_{0}<\infty
\end{aligned}
$$

the last inequality is because of the definition of the spaces $\Omega^{2, r}$. Thus, for any fixed $t$, the map $f_{t}:\left(M, g_{0}\right) \rightarrow\left(M, g_{t}\right)$ is harmonic and satisfies

$$
\int_{M} \rho_{t}^{2}\left(f_{t}, i d\right) d v_{0}<\infty
$$

However, such $f_{t}$ is unique, that is, if there is another map

$$
\bar{f}_{t}:\left(M, g_{0}\right) \rightarrow\left(M, g_{t}\right)
$$

homotopic to $i d$ which is also harmonic and satisfies

$$
\int_{M} \rho_{t}^{2}\left(\bar{f}_{t}, i d\right) d v_{0}<\infty
$$

then we can deduce that $f_{t} \equiv \bar{f}_{t}$. In fact, since $\rho_{t}\left(f_{t}, \bar{f}_{t}\right) \leq \rho_{t}\left(f_{t}, i d\right)+\rho_{t}\left(\bar{f}_{t}, i d\right)$, we have

$$
\int_{M} \rho_{t}^{2}\left(f_{t}, \bar{f}_{t}\right) d v_{0} \leq 2 \int_{M} \rho_{t}^{2}\left(f_{t}, i d\right) d v_{0}+2 \int_{M} \rho_{t}^{2}\left(\bar{f}_{t}, i d\right) d v_{0}<\infty
$$

that is,

$$
\rho_{t}\left(f_{t}, \bar{f}_{t}\right) \in L^{2}\left(M, g_{0}\right)
$$

On the other hand,

$$
\Delta \rho_{t}\left(f_{t}, \bar{f}_{t}\right) \geq-\left(\left|\tau_{t}\left(f_{t}\right)\right|_{g_{t}}+\left|\tau_{t}\left(\bar{f}_{t}\right)\right|_{g_{t}}\right)=0 .
$$

From (4.16) and (4.17) we see that $\rho_{t}\left(f_{t}, \bar{f}_{t}\right)$ is a nonnegative subharmonic function on $\left(M, g_{0}\right)$ which is in $L^{2}\left(M, g_{0}\right)$, by Theorem 3 in [32], we conclude that

$$
\rho_{t}\left(f_{t}, \bar{f}_{t}\right) \equiv \text { const. }
$$

But the volume of $\left(M, g_{0}\right)$ is infinite, this contradicts with $\int_{M} \rho_{t}^{2}\left(f_{t}, \bar{f}_{t}\right) d v_{0}<\infty$. We must have $\rho_{t}\left(f_{t}, \bar{f}_{t}\right) \equiv 0$, consequently, $f_{t} \equiv \bar{f}_{t}$. (Here we would like to remark that having this uniqueness of $f_{t}$, we then have a determined family of $\left\{f_{t}\right\}$ corresponding to $\left\{g_{t}\right\}$.)

Now we consider the map $i d:\left(M, g_{0}\right) \rightarrow\left(M, g_{t}\right)$, a sequence of harmonic maps $\left\{f_{t, i}\right\}$ solving the following

$$
\left\{\begin{array}{l}
\tau_{t}\left(f_{t, i}\right)=0 \\
\left.f_{t, i}\right|_{\partial \Omega_{i}}=\left.i d\right|_{\partial \Omega_{i}}, \quad i=1,2, \cdots
\end{array}\right.
$$

for an exhaustion $\left\{\Omega_{i}\right\}$ of $M$. For these $f_{t, i}$ we have the following estimates

$$
\int_{\Omega_{i}} \rho_{t}^{2}\left(f_{t, i}, i d\right) d v_{0}<\frac{1}{\lambda^{2}(M)}\left\|\tau_{t}(i d)\right\|_{g_{t}, L^{2}\left(M, g_{0}\right)} \leq C_{0},
$$


where $C_{0}>0$ is a constant depending only on $\lambda(M)$ and the curve of metrics $\left\{g_{t}\right\}_{0 \leq t \leq 1}$, but independent of $t$. And $f_{t, i}, i=1,2, \cdots$ converges uniformly on any compact subsets of $M$ to a harmonic map $\hat{f}_{t}:\left(M, g_{0}\right) \rightarrow\left(M, g_{t}\right)$ as $i \rightarrow \infty$, furthermore, from (4.19), $\hat{f}_{t}$ satisfies

$$
\int_{M} \rho_{t}^{2}\left(\hat{f}_{t}, i d\right) d v_{0} \leq C_{0}
$$

(c.f. [3], also [17]). By the above mentioned uniqueness of $f_{t}$, we know that $f_{t} \equiv \hat{f}_{t}$ and consequently

$$
\int_{M} \rho_{t}^{2}\left(f_{t}, i d\right) d v_{0} \leq C_{0} \quad \forall t
$$

This proves (4.3).

5. The local existence of harmonic maps. We are searching for a curve $\left\{f_{t}\right\}_{0 \leq t \leq 1}$ in $\mathrm{comp}^{2, r}(i d)$ such that

$$
\widetilde{F}\left(t, f_{t}\right)=\operatorname{tr}_{g_{0}} \nabla^{g_{t}} d f_{t}=\tau_{t}\left(f_{t}\right)=0 .
$$

$f_{1}$ would be the harmonic map $f_{1}:\left(M^{2}, g_{0}\right) \rightarrow\left(M^{2}, g_{1}\right)$ we are searching for. We will prove in section 8 that $f_{t}$ must be a diffeomorphism. Our main tool is the continuity method. Define

$$
L=\left\{t \in[0,1] \mid \text { There } \quad \text { exists } f \in \Omega^{2}\left(\left(M, g_{0}\right),\left(M, g_{1}\right)\right) \text { s.t. } \tau_{t}(f)=0\right\} .
$$

The uniqueness will be later discussed. the set $L$ is not empty since $f_{0}:\left(M^{2}, g_{0}\right) \rightarrow$ $\left(M^{2}, g_{0}\right)$ is harmonic and $0 \in L$. In this section, we want to show that $L$ is open. For this we use the implicit function technique and express $\tilde{F}$ in local coordinates to get maps in vector spaces.

Let $f_{t_{0}}$ be harmonic, $\mathscr{U}_{\varepsilon}\left(f_{t_{0}}\right)$ a sufficiently small neighborhood of $f_{t_{0}} \in \operatorname{comp}^{2, r}(i d)$ and $\mathscr{U}_{\varepsilon}(0)=\exp _{f_{t_{0}}}^{-1}\left(\mathscr{U}_{\varepsilon}\left(f_{t_{0}}\right)\right) \subset T_{f_{t_{0}}} \operatorname{comp}^{2, r}(i d)$.

Consider the map

$$
\begin{gathered}
\left\{\mathscr{U}_{\delta}\left(t_{0}\right) \cap[0,1]\right\} \times \mathscr{U}_{\varepsilon}(0) \rightarrow T_{f_{t_{0}}} \operatorname{comp}^{2, r-2}(i d) \\
(t, Y) \mapsto F(t, Y),
\end{gathered}
$$

where $\mathscr{U}_{\delta}\left(t_{0}\right):=\left(t_{0}-\delta, t_{0}+\delta\right), F(t, Y)=P_{-Y} t_{g_{0}} \nabla^{g(t)} d \exp Y$ and $P_{-Y}$ is the parallel translation along the geodesic $u \rightarrow \exp u\left(-P_{Y} Y\right.$ ) from $\exp Y$ to $f_{t_{0}}$ (we omit the points $x \in M)$, i.e., $F\left(t_{0}, \cdot\right)$ is a non-linear map from $\mathscr{U}_{\varepsilon}(0) \subset T_{f_{t_{0}}} \operatorname{comp}^{2, r}(i d) \rightarrow$ $T_{f_{t_{0}}} c o m p^{2, r-2}(i d)$.

If we start with $t=0, f_{0}=i d:\left(M^{2}, g_{0}\right) \rightarrow\left(M^{2}, g_{0}\right)$, then

$$
F(0,0)=0 \text {. }
$$

Proposition 5.1. L is open in $[0,1]$. 
Proof. Let $\Delta_{\mathrm{v} f, f} X:=-g_{0}^{i j} \nabla_{i} \nabla_{j} X=-t r_{g_{0}} \nabla^{2} X, X \in \Omega^{2, r}\left(f^{*} T M\right)$ be the rough Laplacian acting on vector fields along $f$. Then

$$
\begin{aligned}
F_{X}(0,0) Y & =\left.\frac{d}{d \tau}\right|_{\tau=0} F(0, \tau Y) \\
& =\left.\frac{d}{d \tau}\right|_{\tau=0} P_{-\tau Y} \operatorname{tr}_{g_{0}} \nabla^{g_{0}} d(i d)+\left.\frac{d}{d \tau}\right|_{\tau=0} \operatorname{tr} r_{g_{0}} \nabla^{g_{0}} d(\exp \tau Y) \\
& =\left.\frac{d}{d \tau}\right|_{\tau=0} t r_{g_{0}} \nabla^{g_{0}} d(\exp \tau Y) \\
& =-\Delta_{\mathrm{v} f, i d} Y+\operatorname{tr}_{g_{0}} R^{g_{0}}(Y, d(i d)) d(i d)
\end{aligned}
$$

The last equation is a straightforward calculation. Here we obtain in our case $n=2$ and $K \equiv-1$

$$
\begin{aligned}
\left\langle F_{X}(0,0) Y, Y\right\rangle_{L^{2}} & =-\left\langle\Delta_{\mathrm{v} f, i d} Y, Y\right\rangle_{L^{2}}+\left\langle\operatorname{Ric}^{g_{0}} Y, Y\right\rangle_{L^{2}} \\
& \leq-\frac{1}{2}|Y|_{L^{2}}^{2}
\end{aligned}
$$

Hence $F_{X}(0,0)$ has zero kernel and bounded inverse with closed image. Using the $\Delta$-based definition of Sobolev spaces, we conclude easily that $F_{X}(0,0)$ is an isomorphism between $T_{i d} c o m p^{2, r}(i d)=\Omega^{2, r}\left(T M, g_{0}\right)$ and $T_{i d} c o m p^{2, r-2}(i d)=$ $\Omega^{2, r-2}\left(T M, g_{0}\right)$ since $F_{X}(0,0)$ is essentially self-adjoint and dimcoker $F_{X}(0,0)=$ $\operatorname{dimker} F_{X}(0,0)=0$.

According to the implicit function theorem, there exist $\delta>0, \varepsilon>o$ such that for $t \in[0, \delta]$ there is a unique $X=X(t) \in \mathscr{U}_{\varepsilon}(0) \subset T_{f_{t_{0}}} \operatorname{comp}^{2, r}(i d)$ such that $F(t, X(t))=0$, i.e.,

$$
\operatorname{tr}_{g_{0}} \nabla^{g(t)} d(\exp X(t))=\tau_{t}(\exp X(t))=0 .
$$

$L$ contains an open neighborhood of $t_{0}=0$ in $[0,1]$. Now let $0 \neq t_{0} \in L, \tau_{t_{0}}\left(f_{t_{0}}\right)=$ $\operatorname{tr}_{g_{0}} \nabla^{g_{t_{0}}} d f_{t_{0}}=0$

$\mathscr{U}_{\delta}\left(t_{0}\right) \times \mathscr{U}_{\varepsilon}(0) \ni(t, Y) \rightarrow P_{-Y} \operatorname{tr}_{g_{0}} \nabla^{g(t)} d(\exp Y)=P_{-Y} \tau_{t}(\exp Y)=F(t, Y)$

with $\mathscr{U}_{\varepsilon} \subset T_{f_{t_{0}}} \operatorname{comp}^{2, r}(i d), Y$ a Sobolev $r$-vector field along $f_{t_{0}}$.

We recall $\nabla \equiv \nabla^{g_{0}}$, $\exp \equiv \exp ^{g_{0}}, R \equiv R^{g_{0}},|\cdot| \equiv|\cdot|$ and $\Gamma \equiv \Gamma^{g_{0}}$, i.e., without " $g_{0}$ " everything is with respect to $g_{0}$. Moreover, we remark that in local normal coordinates $\left\{u^{i}\right\}_{i}$ and $X=\xi^{i} \frac{\partial}{\partial u^{i}}$,

$$
C_{1}|X|_{g, x} \leq\left(\sum_{i}\left(\xi^{i}\right)^{2}\right)^{1 / 2} \equiv|X|_{e u c l} \leq C_{2}|X|_{g, x}
$$

holds for arbitrary vector fields. Then again a straightforward calculation yields

$$
\begin{aligned}
F_{X}\left(t_{0}, 0\right) Y & =\left.\frac{d}{d \tau}\right|_{\tau=0} F\left(t_{0}, \tau Y\right) \\
& =\left.\frac{d}{d \tau}\right|_{\tau=0} P_{-\tau Y} t r_{g_{0}} \nabla^{g_{t_{0}}} d f_{t_{0}}+\left.\frac{d}{d \tau}\right|_{\tau=0} t r_{g_{0}} \nabla^{g_{0}} d(\exp \tau Y) \\
& =\left.\frac{d}{d \tau}\right|_{\tau=0}\left[\operatorname{tr}_{g_{0}} \nabla^{g_{t_{0}}} d(\exp \tau Y)\right] \\
& =-\Delta_{\mathrm{v} f, f_{t_{0}}} Y+\operatorname{tr}_{g_{0}} R^{g_{t_{0}}}\left(Y, d f_{t_{0}}\right) d f_{t_{0}}
\end{aligned}
$$




$$
\begin{aligned}
\left\langle F_{X}\left(t_{0}, 0\right) Y, Y\right\rangle_{L^{2}}= & -\left\langle\Delta_{\mathrm{v} f, f_{t_{0}}} Y, Y\right\rangle_{L^{2}} \\
& +\int_{M}\left\langle\operatorname{tr}_{g_{0}} R^{g_{t_{0}}}\left(Y, d f_{t_{0}}\right) d f_{t_{0}}, Y\right\rangle_{f_{t_{0}}^{*} g_{t_{0}}} d \operatorname{vol}_{x}\left(f_{t_{0}}^{*} g_{t_{0}}\right),
\end{aligned}
$$

where all scalar products in (5.4) are taken in $\left(f_{t_{0}}^{*} T M, f_{t_{0}}^{*} g_{t_{0}}\right)$.

We recall our main assumption $\inf \sigma_{e}\left(\Delta_{0}\left(g_{0}\right)\right)>0, \operatorname{comp}^{2, r}\left(g_{0}\right) \subset \mathscr{M}^{2, r}\left(I, B_{\infty}\right)$, $r>3, r_{i n j}\left(g_{0}\right)>0$, in particular, $\operatorname{vol}\left(M^{2}, g_{0}\right)=\operatorname{vol}\left(M^{2}, g_{t}\right)=\infty, K_{g_{t}} \equiv-1$.

The assumption $\inf \sigma_{e}\left(\Delta_{0}\left(g_{0}\right)\right)>0,\left\{g_{t}\right\}_{0 \leq t \leq 1}$ a smooth curve in comp $\left(g_{0}\right)_{-1} \subset$ $c o m p^{2, r}\left(g_{0}\right)$ imply inf $\sigma_{e}\left(\Delta_{0}\left(g_{t}\right)\right)=\inf \sigma_{e}\left(\Delta_{0}\left(g_{0}\right)\right)>0$. Hence below $\inf \sigma_{e}\left(\Delta_{0}\left(g_{t}\right)\right)$ there can be only discrete nonnegative eigenvalues of finite multiplicity which cannot accumulate at 0 . Moreover, 0 is not an eigenvalue of $\Delta_{0}\left(g_{t}\right)$ since $\operatorname{vol}\left(M^{2}, g_{t}\right)=\infty$. Hence if inf $\sigma_{e}\left(\Delta_{0}\left(g_{t}\right)\right)>0$, for compactness reasons $\inf _{t \in[0,1]} \inf \sigma_{e}\left(\Delta_{0}\left(g_{t}\right)\right)=\lambda_{0}>0$.

We remark that for any Riemannian vector bundle $(E, h, \nabla)$ over a complete Riemannian manifold $\left(M^{2}, g\right)$ there holds that $\phi \in C_{c}^{\infty}(E)$ implies $|\phi| \in \Omega^{0,2,1}\left(M^{n}, g\right)$. Moreover,

$$
\begin{aligned}
\inf \sigma\left(\Delta_{0}\left(M^{n}, g\right)\right) & =\inf _{0 \neq \psi \in C_{c}^{\infty}(M)} \frac{|\nabla \psi|_{L^{2}}^{2}}{|\psi|_{L^{2}}^{2}} \\
& =\inf _{0 \neq \chi \in \Omega^{0,2,1}(M)} \frac{|\nabla \chi|_{L^{2}}^{2}}{|\chi|_{L^{2}}^{2}} .
\end{aligned}
$$

Lemma 5.2. Let $\left(M^{n}, g\right)$ be open, complete, $\operatorname{vol}\left(M^{n}, g\right)=\infty, \lambda_{0, M}=$ $\inf \sigma\left(\Delta_{0}(g)\right)>0$ and let $(E, h, \nabla) \rightarrow\left(M^{n}, g\right)$ be a Riemannian vector bundle over $M$. If $\lambda_{0, E}=\inf \sigma\left(\nabla^{*} \nabla\right)$, then

$$
\lambda_{0, E} \geq \lambda_{0, M}
$$

Proof. According to (5.5) and Kato's inequality $|d| \phi||_{x} \leq|\nabla \phi|_{x}$,

$$
\begin{aligned}
\lambda_{0, M} & =\inf _{0 \neq \chi \in \Omega^{0,2,1}(M)} \frac{|\nabla \chi|_{L^{2}}^{2}}{|\chi|_{L^{2}}^{2}} \\
& \leq \inf _{0 \neq \phi \in C_{c}^{\infty}(E)} \frac{\left.|d| \phi\right|_{L^{2}} ^{2}}{|\phi|_{L^{2}}^{2}} \\
& \leq \inf _{0 \neq \phi \in C_{c}^{\infty}(E)} \frac{|\nabla \phi|_{L^{2}}^{2}}{|\phi|_{L^{2}}^{2}}=\lambda_{0, E} .
\end{aligned}
$$

We now apply Lemma 5.2 to $E=f_{t_{0}}^{*} T M, \nabla^{*} \nabla=\Delta_{\mathrm{v} f, f_{t_{0}}}$ and obtain from (5.6) and $\left\langle\operatorname{tr}_{g_{0}} R^{g_{t_{0}}}\left(Y, d f_{t_{0}}\right) d f_{t_{0}}, Y\right\rangle_{g_{t_{0}}} \leq 0$ that

$$
\left.\left\langle F_{X}\left(t_{0}, 0\right) Y, Y\right\rangle_{L^{2}} \leq-C|Y|_{L^{2}}^{2}, \quad 0<\inf _{t \in[0,1]} \inf \sigma \Delta_{0}\left(g_{t}\right)\right) \leq C .
$$

From this we immediately see that $F_{X}\left(t_{0}, 0\right): \Omega^{2, r}\left(f_{t_{0}}^{*} T M\right) \rightarrow \Omega^{2, r-2}\left(f_{t_{0}}^{*} T M\right)$ is an isomorphism: $\operatorname{ker} \Delta_{\mathrm{v} f, f_{t_{0}}}=\{0\}, \operatorname{Im}\left(\Delta_{\mathrm{v} f, f_{t_{0}}}\right)$ is closed, and $\operatorname{ker} \Delta_{\mathrm{v} f, f_{t_{0}}}^{*}=\{0\}$. Applying again the implicit function theorem, there exist $\delta>0, \varepsilon>0$ such that 
for $t \in \mathscr{U}_{\delta}\left(t_{0}\right) \cap[0,1]$ there is a unique $Y=Y(t) \in \mathscr{U}_{\varepsilon}(0) \subset \Omega^{2, r}\left(f_{t_{0}}^{*} T M\right)$ with $F(t, Y(t))=0$, i.e., $\tau_{t}(\exp Y(t))=0$. We conclude that $L$ is open in $[0,1]$, the proof of Proposition 5.1 is done.

We infer from the implicit function theorem even more. The map

$$
\mathscr{U}\left(t_{0}, 0\right) \ni(t, Y) \rightarrow F(t, Y)=P_{-Y} t_{g_{0}} d(\exp Y)
$$

is smooth since $\left\{g_{t}\right\}_{0 \leq t \leq 1}$ is smooth. Then, according to the implicit function theorem, the function $t \rightarrow Y(t)$ is smooth and hence $t \rightarrow \exp Y(t)$ is a smooth curve in $c o m p^{2, r}(i d)$. The derivative $\frac{\partial}{\partial t} Y(t)$ is given by

$$
\frac{\partial}{\partial t} Y(t)=-F_{X}(t, Y(t))^{-1} F_{t}(t, Y(t))
$$

An explicit expression for the vector field $\xi_{t}(x)=\frac{\partial}{\partial t} \exp _{x} Y(t)$ can be obtained as follows. $c(s . t)=\exp (s Y(t))$ defines a Jacobi field $J_{t}(s)=\frac{\partial}{\partial t} \exp (s Y(t)), \xi=$ $\frac{\partial}{\partial t} \exp Y(t)=J_{t}(1), J_{t}(0)=0, \nabla_{s} J_{t}(s)=\nabla_{t} Y(t)$, hence

$$
\xi=\frac{\partial}{\partial t} \exp Y(t)=\left(d\left(\exp _{f_{t_{0}}} Y(t)\right)\left(\nabla_{\frac{\partial}{\partial t}} Y(t)\right) .\right.
$$

An estimate for $\left|\xi_{t}\right|$ plays an essential role. In [22] has been established an elliptic PDE for $\xi$ which could be use to estimate $\left|\xi_{t}\right|$ but we have chosen in section 4 another approach. To prove $L=[0,1]$, we must show the following. Assume that $t_{1}<t_{2}<$ $\cdots<t_{0}, t_{\nu} \in L, \nu \geq 1, t_{\nu} \rightarrow t_{0}$, then $t_{0} \in L$. The canonical procedure to prove this would be to prove

$$
\begin{gathered}
\left(f_{t_{\nu}}\right)_{\nu} \text { is a Cauchy sequence in } \operatorname{comp}^{2, r}(i d), \\
f_{t_{\nu}} \rightarrow f_{t_{0}} \\
\tau\left(f_{t_{0}}\right)=0 .
\end{gathered}
$$

As well known from other treatises on harmonic maps, the proof of (5.8), (5.10) would be the hardest part of an existence theorem for harmonic maps. In the next section, we shall follow another road which will turn out as essentially equivalent to (5.8) and (5.10).

6. The reduction to a uniform Banach fixed point theorem. The proof of $L=[0,1]$ will immediately follow from the following

TheOrem 6.1. The exists a $\delta>0$ independent of $t_{0}$ such that

$$
\left(t_{0}-\delta, t_{0}+\delta\right) \cap[0,1] \subset L .
$$

We shall later see that the proof of (6.1) is equivalent to (5.8)-(5.10).

The proof of Theorem 6.1 essentially relies on careful estimates of $F_{X}(t, Y)$ and $F(t, Y)$ to which we turn our attention in the next section. 
First we sketch the key idea proof of Theorem 6.1.

Let $t_{0} \in L, f_{t_{0}}:\left(M^{2}, g_{0}\right) \rightarrow\left(M^{2}, g_{t_{0}}\right), f_{t_{0}} \in \operatorname{comp}^{2, r}(i d) \subset \Omega^{2 r}(M, M), \tau_{t_{0}}\left(f_{t_{0}}\right)=$ $t r_{g_{0}} \nabla^{g_{t_{0}}} d f_{t_{0}}=0$ and set with $F(t, Y)=P_{-Y} t r_{g_{0}} \nabla^{g(t)} d \exp Y$

$$
\begin{aligned}
g(t, Y) & :=F_{X}\left(t_{0}, 0\right) Y-F(t, Y) \\
& =\left.\frac{d}{d \tau}\right|_{\tau=0}\left[P_{-\tau Y} t_{g_{0}} \nabla^{g_{t_{0}}} d(\exp \tau Y)\right]-F(t, Y)
\end{aligned}
$$

for $Y \in \mathscr{U}_{\varepsilon}(0) \subset T_{f_{t_{0}}} \operatorname{comp}^{2, r}(i d) \subset \Omega^{2 . r}\left(f_{t_{0}}^{*} T M\right), \varepsilon$ sufficiently small. $g$ is a map

$$
[0,1] \times \mathscr{U}_{\varepsilon}(0) \rightarrow T_{f_{t_{0}}} \operatorname{comp}^{2, r-2}(i d) .
$$

Then $\tau_{t}(\exp Y)=0$ if and only if $F(t, Y)=0$ if and only if $Y=F_{X}\left(t_{0}, 0\right)^{-1} g(t, Y)$. Hence if we set

$$
T_{t} Y:=F_{X}\left(t_{0}, 0\right)^{-1} g(t, Y),
$$

then

$$
F(t, Y)=0 \text { if and only if } T_{t} Y=Y,
$$

i.e., if $Y$ is a fixed point of $\tau_{t}$. Hence we would be done if there would exist a $\delta>0$ and for every $t_{0} \in L$ a (non-empty) complete metric space $M_{t_{0}, \delta} \subset T_{f_{t_{0}}} c o m p^{2, r}(i d)$ such that

$$
T_{t}: M_{t_{0}} \rightarrow M_{t_{0}}
$$

and

$$
T_{t} \quad \text { is contracting for all } t \in\left(t_{0}-\delta, t_{0}+\delta\right) \cap[0,1] \text {, }
$$

where $\delta$ is independent of $t_{0}$.

To establish (6.5) and (6.6), we must carefully estimate the operator norm $\left|F_{X}\left(t_{0}, 0\right)^{-1}\right|_{r-2, r}$ and the Sobolev norm $|g(t, Y)|_{2, r-2}$.

We write

$$
T_{t} Y:=F_{X}\left(t_{0}, 0\right)^{-1} g(t, Y)=F_{X}\left(t_{0}, 0\right)^{-1}[g(t, Y)-g(t, 0)+g(t, 0)] .
$$

Proposition 6.2.

1. $\left|F_{X}\left(t_{0}, 0\right)^{-1}\right|_{r-2, r} \leq C_{1}, \quad C_{1}$ independent of $t_{0}$.

2. $|g(t, 0)|_{r-2} \equiv|g(t, 0)|_{2, r-2} \leq C_{2}\left|t-t_{0}\right|, \quad C_{2}$ independent of $t_{0}$.

3. $|g(t, Y)-g(t, 0)|_{r-2} \leq o_{1}(1)|Y|_{r}$, where $o_{1}(1) \rightarrow 0$ as $|Y|_{r} \rightarrow 0$ and $\left|t-t_{0}\right| \rightarrow 0$ with rate independent of $t_{0}$.

4. $\left|g\left(t, Y_{1}\right)-g\left(t, Y_{2}\right)\right|_{r-2} \leq o_{2}(1)\left|Y_{1}-Y_{2}\right|_{r}$, where $o_{2}(1) \rightarrow 0$ as $\left|Y_{1}\right|_{r} \rightarrow 0$ and $\left|Y_{2}\right|_{r} \rightarrow 0$ with rate independent of $t_{0}$. 
The proof of (6.8)-(6.11) will occupy the whole next section.

Now we draw our conclusions from Proposition 6.2. Choose $\rho$ so small such that

$$
o_{i}(1) \leq \frac{1}{2 C_{1}}
$$

for $|Y|_{r} \leq \rho$ and $\left|t-t_{0}\right| \leq \delta, i=1,2$. Then

$$
\begin{aligned}
& \mid F_{X}\left(t_{0}, 0\right)^{-1}\left[\left.(g(t, Y)-g(t, 0)+g(t, 0)]\right|_{r}\right. \\
& \leq C_{1}\left[o_{1}(1)|Y|_{r}+C_{2}\left|t-t_{0}\right|\right] \\
& \leq C_{1}\left[\frac{1}{2 C_{1}}|Y|_{r}+C_{2}\left|t-t_{0}\right|\right] \\
& \leq \frac{1}{2}|Y|_{r}+C_{1} C_{2}\left|t-t_{0}\right| \\
& \leq \frac{1}{2} \rho+C_{1} C_{2}\left|t-t_{0}\right| \\
& \leq \frac{1}{2} \rho+\frac{1}{2} \rho=\rho
\end{aligned}
$$

for $\left|t-t_{0}\right| \leq \frac{\rho}{2 C_{1} C_{2}}$.

Hence

$$
T_{t}: M_{\rho} \rightarrow M_{\rho}, \quad M_{\rho}=\left\{\left.Y \in \Omega^{2, r}\left(f_{t_{0}}^{*} T M\right)|| Y\right|_{r} \leq \rho\right\}
$$

Theorem 6.3. $T_{t}: M_{\rho} \rightarrow M_{\rho}$ is contractive for $\left|t-t_{0}\right| \leq \min \left\{\delta, \frac{\rho}{2 C_{1} C_{2}}\right\}$.

Proof. Let $Y_{1}, Y_{2} \in M_{\rho}$. Then

$$
\begin{aligned}
\left|T_{t} Y_{1}-T_{t} Y_{2}\right|_{r} & \leq C_{1} o_{2}(1)\left|Y_{1}-Y_{2}\right|_{r} \\
& \leq C_{1} \frac{1}{2 C_{1}}\left|Y_{1}-Y_{2}\right|_{r} \\
& =\frac{1}{2}\left|Y_{1}-Y_{2}\right|_{r} .
\end{aligned}
$$

7. Proof of the existence theorem. As we pointed out in the preceding section, the proof of the existence theorem essentially reduces to the proof of Proposition 6.2. We start with the uniform boundedness of $F_{X}\left(t_{0}, 0\right)^{-1}$. Let $f_{t_{0}}:\left(M^{2}, g_{0}\right) \rightarrow$ $\left(M^{2}, g_{t_{0}}\right) \in \operatorname{comp}(i d) \subset \Omega^{2 . r}(M, M)$ be harmonic, where $\left\{g_{t}\right\}_{0 \leq t \leq 1}$ is the given curve. Then, according to (5.3),

$$
F_{X}\left(t_{0}, 0\right) Y=-\Delta_{\mathrm{v} f, f_{t_{0}}} Y+t r_{g_{0}} R^{g_{t_{0}}}\left(Y, d f_{t_{0}}\right) d f_{t_{0}} .
$$

Lemma 7.1. There exists $C_{r-2, r}>0$, independent of $t_{0}$, such that

$$
\left|F_{X}\left(t_{0}, 0\right)^{-1}\right|_{\Omega^{2, r-2} \rightarrow \Omega^{2, r}} \equiv\left|F_{X}\left(t_{0}, 0\right)^{-1}\right|_{r-2, r} \leq C_{r-2, r} .
$$

Proof. We know from (5.7) and the spectral theorem that

$$
\left|F_{X}\left(t_{0}, 0\right)^{-1}\right|_{0,2} \leq C_{0,2} \text {. }
$$


Write

$$
F_{X}\left(t_{0}, 0\right) Y=-\Delta_{\mathrm{v} f, f_{t_{0}}} Y+t r_{g_{0}} R^{g_{t_{0}}}\left(Y, d f_{t_{0}}\right) d f_{t_{0}} \equiv\left(-\Delta_{t_{0}}+\mathscr{R}_{t_{0}}\right) Y .
$$

Then (7.2) is equivalent to

$$
\left|\left(-\Delta_{t_{0}}+\mathscr{R}_{t_{0}}\right)^{-1} Y\right|_{2,0} \leq C_{0,2}|Y|_{2,0} .
$$

Here and in the sequel, for a vector field $Z$, denote $|Z|_{2, i}$ the i-th Sobolev norm for $p=2$, in particular, $|Z|_{2,0}:=|Z|_{L^{2}}$. According to Theorem 2.12, (7.1) would be done if we could additionally to (7.2) establish

$$
\left|\left(-\Delta_{t_{0}}\right)^{i}\left(-\Delta_{t_{0}}+\mathscr{R}_{t_{0}}\right)^{-1} Y\right|_{2,0} \leq C_{i}|Y|_{2,2(i-1)}, \quad 1 \leq i \leq \frac{r}{2} .
$$

Let $i=1$, then

$$
\begin{aligned}
\left|\left(-\Delta_{t_{0}}\right)\left(-\Delta_{t_{0}}+\mathscr{R}_{t_{0}}\right)^{-1} Y\right|_{2,0} & =\left|Y-\mathscr{R}_{t_{0}}\left(-\Delta_{t_{0}}+\mathscr{R}_{t_{0}}\right)^{-1} Y\right|_{2,0} \\
& \leq|Y|_{2,0}+d_{1}|Y|_{2,0}=\left(1+d_{1}\right)|Y|_{2,0}=C_{1}|Y|_{2,0} .
\end{aligned}
$$

Using (4.10), Theorem 2.12, $\left(B_{\infty}\right)$ and the Leibniz rule, we immediately obtain for $i \leq \frac{r}{2}$ that

$$
\begin{aligned}
& \left|\left(-\Delta_{t_{0}}\right)^{i}\left(-\Delta_{t_{0}}+\mathscr{R}_{t_{0}}\right)^{-1} Y\right|_{2,0} \\
& =\left|\left(-\Delta_{t_{0}}\right)^{i-1} Y-\left(-\Delta_{t_{0}}\right)^{i-1} \mathscr{R}_{t_{0}}\left(-\Delta_{t_{0}}+\mathscr{R}_{t_{0}}\right)^{-1} Y\right|_{2,0} \\
& \leq d_{2(i-1)}|Y|_{2,2(i-1)}+\sum_{j-0}^{2(i-1)} d_{j}^{\prime}|Y|_{2, j} \leq C_{i}|Y|_{2,2(i-1)},
\end{aligned}
$$

where $C_{i}$ is independent of $t_{0}$.

Proposition 7.2. Under the assumptions above, there holds

$$
|g(t, 0)|_{2, r-2} \leq C\left|t-t_{0}\right|=C_{2}\left|t-t_{0}\right| .
$$

Proof. We first note that

$$
\begin{aligned}
g(t, 0)= & -F(t, 0)=F\left(t_{0}, 0\right)-F(t, 0)=t r_{g_{0}} \nabla^{g_{t_{0}}} d f_{t_{0}} \\
& -\operatorname{tr}_{g_{0}} \nabla^{g_{t}} d f_{t_{0}}=t r_{g_{0}}\left[\left(\nabla^{g_{t_{0}}}-\nabla^{g_{t}}\right) d f_{t_{0}}\right] .
\end{aligned}
$$

Now we give an expression for $\nabla^{g_{t_{0}}}-\nabla^{g_{t}}$.

Lemma 7.3. Let $g$ be a $C^{1}$-metric, $A$ a symmetric tensor field such that $g+A$ is still $C^{1}$-metric. Then there holds in local coordinates

$$
\Gamma(g+A)_{j k}^{i}=\Gamma(g)_{j k}^{i}+\frac{1}{2}\left((g+A)^{-1}\right)^{i l}\left(A_{l j ; k}+A_{l k ; j}+A_{j k ; l}\right),
$$

where; is the covariant derivative with respect to $g$.

Proof. We refer to [13] for the proof. 
Now we apply this to

$$
\begin{gathered}
g_{t, i j}-g_{t_{0}, i j}=\dot{g}_{t, i j}\left(t_{0}+\theta_{i j}\left(t-t_{0}\right)\right)\left(t-t_{0}\right), \quad 0<\theta_{i j}<1, \\
\left(g_{t, i j}\right)=\left(g_{t_{0}, i j}+A_{i j}\right)
\end{gathered}
$$

with

$$
\left\{\begin{aligned}
\left(A_{i j}\right) & =\left(\dot{g}_{t, i j}\left(t_{0}+\theta_{i j}\left(t-t_{0}\right)\right)\right)\left(t-t_{0}\right)=\left(B_{i j}\right)\left(t-t_{0}\right), \\
\left(g_{t, i j}\right) & =\left(g_{t_{0}, i j}\right)+\left(B_{i j}\right)\left(t-t_{0}\right),
\end{aligned}\right.
$$

and according to (7.4)-(7.5), we get

$$
\Gamma_{j k}^{i}\left(g_{t}\right)-\Gamma_{j k}^{i}\left(g_{t_{0}}\right)=\frac{1}{2} g_{t}^{i l}\left(B_{l j ; k}+B_{l k ; j}+B_{j k ; l}\right)\left(t-t_{0}\right) .
$$

Here we take; with respect to $g_{t_{0}}$. This yields in local coordinates $\left(x^{1}, x^{2}\right)$ about $x_{0} \in M^{2}$ and $\left(y^{1}, y^{2}\right)$ about $f_{t_{0}}\left(x_{0}\right)$

$$
\begin{aligned}
\left(\nabla^{g_{t}}-\nabla^{g_{0}}\right) \frac{\partial}{\partial x^{s}} d f_{t_{0}}= & \left(\Gamma_{i j}^{k}\left(g_{t}\right)-\Gamma_{i j}^{k}\left(g_{t_{0}}\right)\right) \frac{\partial f_{t_{0}}^{i}}{\partial x^{r}} d x^{r} \otimes \frac{\partial f_{t_{0}}^{j}}{\partial x^{s}} \frac{\partial}{\partial y^{k}} \\
& +\left(\Gamma_{s j}^{k}\left(g_{t_{0}}\right)-\Gamma_{s j}^{k}\left(g_{t}\right)\right)\left(d x^{j} \otimes \frac{\partial f_{t_{0}}^{i}}{\partial x^{k}} \frac{\partial}{\partial y^{i}}\right)
\end{aligned}
$$

and consequently,

$$
\begin{aligned}
\operatorname{tr}_{g_{0}}\left(\nabla^{g_{t}}-\nabla^{g_{t_{0}}}\right) d f_{t_{0}}= & {\left[g_{0}^{r s}\left(\Gamma_{i j}^{k}\left(g_{t}\right)-\Gamma_{i j}^{k}\left(g_{t_{0}}\right)\right) \frac{\partial f_{t_{0}}^{i}}{\partial x^{r}} \frac{\partial f_{t_{0}}^{j}}{\partial x^{s}}\right] \frac{\partial}{\partial y^{k}} } \\
& +g_{0}^{s j}\left(\Gamma_{s j}^{k}\left(g_{t_{0}}\right)-\Gamma_{s j}^{k}\left(g_{t}\right)\right) \frac{\partial f_{t_{0}}^{i}}{\partial x^{k}} \frac{\partial}{\partial y^{i}} \\
= & \frac{1}{2}\left[g_{0}^{r s}\left(g_{t}^{k l}\left(B_{l i ; j}+B_{l j ; i}+B_{i j ; l}\right) \frac{\partial f_{t_{0}}^{i}}{\partial x^{r}} \frac{\partial f_{t_{0}}^{j}}{\partial x^{s}}\right]\left(t-t_{0}\right) \frac{\partial}{\partial y^{k}}\right. \\
& +\frac{1}{2}\left[g_{0}^{s j} g_{t}^{k l}\left(B_{l s ; j}+B_{l j ; s}+B_{s j ; l}\right) \frac{\partial f_{t_{0}}^{i}}{\partial x^{k}}\right]\left(t_{0}-t\right) \frac{\partial}{\partial y^{i}} .
\end{aligned}
$$

By assumption, $g_{t} \in \operatorname{comp}_{-1}^{2 . r}\left(g_{0}\right), 0 \leq t \leq 1$, which implies

$$
\left|g_{t}-g_{t_{0}}\right|_{2, r} \leq C_{1}, \quad\left|B_{i j ; k}\right|_{2, r-1} \leq C_{1}^{\prime}
$$

with $C_{1}, C_{1}^{\prime}$ independent of $t, t_{0}$. Moreover, according to (4.10), $\left|\nabla^{i} d f_{t_{0}}\right| \leq d_{i}, 0 \leq i \leq$ $r-1$, and we obtain with (7.7)

$$
|g(t, 0)|_{2, r-1}=|-F(t, 0)|_{2, r-1} \leq C_{2}\left|t-t_{0}\right|,
$$

with $C_{2}$ independent of $t, t_{0}$. $\square$

Finally we estimate $g(t, Y)-g(t, 0)$ and $g\left(t, Y_{1}\right)-g\left(t, Y_{2}\right)$, i.e. we establish (6.10).

Proposition 7.4. Suppose $f_{t_{0}}:\left(M^{2}, g_{0}\right) \rightarrow\left(M^{2}, g_{t_{0}}\right)$ harmonic. Then there holds for $Y \in T_{f_{t_{0}}} \subset \operatorname{comp}^{2, r}(i d) \subset \Omega^{2, r}\left(f_{t_{0}}^{*} T M\right),|Y|<\frac{1}{2} \inf _{t} r_{i n j}\left(g_{t}\right),|Y|_{r}<\frac{1}{2}$ that

$$
|g(t, Y)-g(t, 0)|_{r-2} \leq o_{1}(1)|Y|_{r}, \quad o_{1}(1) \underset{|Y|_{r} \rightarrow 0}{\stackrel{\left|t-t_{0}\right| \rightarrow 0}{\longrightarrow} 0} 0
$$


with rate independent of $t_{0}$.

Proof. Let $V$ and $W$ be Banach spaces, $U \subset V$ open, $f: U \rightarrow W$ a $C^{1}$-map. Then we have the standard estimate

$$
|f(u+h)-f(u)| \leq \sup _{0<\theta<1}\left|\frac{d}{d \tau}\right|_{\tau=0} f(u+(\theta+\tau) h) \mid .
$$

In our case, $f(u)=g(t, Y), V_{t}=\{t\} \times T_{f_{t_{0}}} \operatorname{comp}^{2, r}(i d), W=T_{f_{t_{0}}} \operatorname{comp} p^{2, r-2}(i d)$,

$$
\begin{aligned}
g(t, Y) & =F_{X}\left(t_{0}, 0\right) Y-P_{-Y} t_{g_{0}} \nabla^{g_{t}} d(\exp Y) \\
& =-\Delta_{\mathrm{v} f, f_{t_{0}}} Y+\operatorname{tr}_{g_{0}} R^{g_{t_{0}}}\left(Y, d f_{t_{0}}\right) d f_{t_{0}}-P_{-Y} \operatorname{tr}_{g_{0}} \nabla^{g_{t}} d(\exp Y) .
\end{aligned}
$$

Hence

$$
\begin{aligned}
& \left|\frac{d}{d \tau}\right|_{\tau=0}\left(g \left(t,\left.(\theta+\tau) Y\right|_{r-2}\right.\right. \\
= & \left.\left|\frac{d}{d \tau}\right|_{\tau=0}\left[F_{X}\left(t_{0}, 0\right)(\theta+\tau) Y-P_{-(\theta+\tau) Y} t r_{g_{0}} \nabla^{g_{t}} d(\exp (\theta+\tau) Y)\right]\right|_{r-2} \\
= & \left.\left|F_{X}\left(t_{0}, 0\right) Y-\frac{d}{d s}\right|_{s=\theta}\left[P_{-s Y} \operatorname{tr}_{g_{0}} \nabla^{g_{t}} d(\exp s Y)\right]\right|_{r-2} .
\end{aligned}
$$

We calculate the pointwise expression of the second term.

Consider $c(s)=\exp s Y, P_{-s Y}$ the parallel transport from the point $\exp s Y$ back to the initial point, $0 \leq s \leq 1, s_{0} \in(0,1)$ and let $X(s)$ be a vector field along $c(\cdot)$. Then

$$
\begin{aligned}
\left.\frac{d}{d s}\right|_{s=s_{0}} P_{-s Y} X(s) & =\lim _{s \rightarrow s_{0}} \frac{P_{-s Y} X(s)-P_{-s_{0} Y} X\left(s_{0}\right)}{s-s_{0}} \\
& =\lim _{s \rightarrow s_{0}} \frac{P_{-s_{0} Y} P_{-s Y \rightarrow-s_{0} Y} X(s)-P_{-s_{0} Y} X\left(s_{0}\right)}{s-s_{0}} \\
& =P_{-s_{0} Y} \lim _{s \rightarrow s_{0}} \frac{P_{-s Y \rightarrow-s_{0} Y} X(s)-X\left(s_{0}\right)}{s-s_{0}} \\
& =P_{-s_{0} Y} \nabla_{\dot{c}} X\left(s_{0}\right) .
\end{aligned}
$$

We obtain in our case $\left.\frac{d}{d \tau}\right|_{\tau=0}=\left.\frac{d}{d s}\right|_{s=\theta}$ that

$$
\left.\frac{d}{d \tau}\right|_{\tau=0}\left[P_{-(\theta+\tau) Y} t r_{g_{0}} \nabla^{g_{t}} d(\exp (\theta+\tau) Y)\right]=P_{-\theta Y} \nabla_{c^{\prime}} t r_{g_{0}} \nabla^{g_{t}} d(\exp \theta Y),
$$

where, according to our convention before Proposition 3.6, $\nabla_{c^{\prime}} \equiv \nabla_{c^{\prime}}^{g_{0}}$. In the sequel we always denote $\nabla \equiv \nabla^{g_{0}}, R \equiv R^{g_{0}}$. Then

$$
\begin{aligned}
& \nabla_{c^{\prime}} t_{g_{0}} \nabla^{g_{t}} d(\exp \theta Y) \\
= & \operatorname{tr}_{g_{0}} \nabla_{c^{\prime}} \nabla^{g_{t}} d(\exp \theta Y) \\
= & g_{0}^{i j} \nabla_{c^{\prime}}\left(\nabla^{g_{t}}\right)_{j}(d(\exp \theta Y))\left(e_{i}\right) \\
= & g_{0}^{i j} \nabla_{c^{\prime}}\left(\nabla_{j}(d(\exp \theta Y))\left(e_{i}\right)+g_{0}^{i j} \nabla_{c^{\prime}}\left(\nabla^{g_{t}}-\nabla^{g_{0}}\right)_{j}(d(\exp \theta Y))\left(e_{i}\right)\right. \\
= & g_{0}^{i j} \nabla_{j} \nabla_{c^{\prime}}\left((d(\exp \theta Y))\left(e_{i}\right)+g_{0}^{i j} R^{g_{0}}\left(c^{\prime},(d(\exp \theta Y))\left(e_{j}\right)\right)(d(\exp \theta Y))\left(e_{i}\right)\right. \\
& +g_{0}^{i j} \nabla_{c^{\prime}}\left(\nabla^{g_{t}}-\nabla^{g_{0}}\right)_{j}(d(\exp \theta Y))\left(e_{i}\right) \\
= & g_{0}^{i j} \nabla_{j} \nabla_{i} c^{\prime}+g_{0}^{i j} R^{g_{0}}\left(c^{\prime},(d(\exp \theta Y))\left(e_{j}\right)\right)(d(\exp \theta Y))\left(e_{i}\right) \\
& +g_{0}^{i j} \nabla_{c^{\prime}}\left(\nabla^{g_{t}}-\nabla^{g_{0}}\right)_{j}(d(\exp \theta Y))\left(e_{i}\right),
\end{aligned}
$$


where we used $\nabla_{s} \frac{\partial}{\partial t} c(s, t)=\nabla_{t} \frac{\partial}{\partial s} c(s, t)$.

Hence, with $c^{\prime}=P_{\theta Y} Y$, we have

$$
\begin{aligned}
& F_{X}\left(t_{0}, 0\right) Y-\left.\frac{d}{d s}\right|_{s=\theta} P_{-s Y} t_{g_{0}} \nabla^{g_{t}} d(\exp s Y)=-\Delta_{f_{t_{0}}} Y+\operatorname{tr}_{g_{0}} R^{g_{t_{0}}}\left(Y, d f_{t_{0}}\right) d f_{t_{0}} \\
& +P_{-\theta Y} \Delta_{\exp \theta Y} P_{\theta Y} Y-P_{-\theta Y}\left[\operatorname{tr}_{g_{0}} R^{g_{0}}\left(P_{\theta Y} Y, d(\exp \theta Y)\right) d(\exp \theta Y)\right. \\
& \left.-t r_{g_{0}} \nabla c^{\prime}\left(\nabla^{g_{t}}-\nabla^{g_{0}}\right)(d(\exp \theta Y))\right]
\end{aligned}
$$

Now we start with the estimate of

$$
\Delta_{f_{t_{0}}} Y-P_{-\theta Y} \Delta_{\exp \theta Y} P_{\theta Y} Y
$$

Writing $\Delta \equiv \Delta_{\mathrm{v} f, f_{t_{0}}}, P \equiv P_{\theta Y}, P^{-1} \equiv P_{-\theta Y}$, we have to estimate $\mid(\Delta-$ $\left.P^{-1} \Delta P\right)\left.Y\right|_{r-2}$ and start with the estimate of the pointwise norm $\left|\Delta-P^{-1} \Delta P\right|$ in local coordinates. For this, we recall the standard formula for parallel transport. Let $c(s),-\varepsilon \leq s \leq \varepsilon$ be a $C^{1}$-curve, $X(s)$ a parallel $C^{1}$-vector field along $c$, denote $\dot{c} \equiv \dot{u}^{i} \frac{\partial}{\partial u^{i}}, X \equiv \xi^{j} \frac{\partial}{\partial u^{j}}$. Then, the equation $\nabla_{\dot{c}} X=0$ is equivalent to

$$
\left(\dot{u}^{i} \frac{\partial}{\partial u^{i}} \xi^{k}+\dot{u}^{i} \xi^{l} \Gamma_{i l}^{k}\right) \frac{\partial}{\partial u^{k}}=\left(\frac{d}{d s} \xi^{k}+\dot{u}^{i} \Gamma_{i l}^{k} \xi^{l}\right) \frac{\partial}{\partial u^{k}}=0 .
$$

We can rewrite (7.14) as

$$
\frac{d}{d s} X(s)=-\left\langle\dot{c}, \Gamma^{o p} X\right\rangle_{e u c l},
$$

where $\Gamma^{o p} X:=\Gamma_{i l}^{k} \xi^{l}$ and $\langle,\rangle_{\text {eucl }}$ means the euclidean scalar product in local coordinates which in normal coordinates is equivalent to the Riemannian one since we have bounded geometry.

We now perform the estimate of $\left|\left(\Delta-P^{-1} \Delta P\right) Y\right|$ in normal coordinates.

$$
\begin{aligned}
\left|P_{-\theta Y} \Delta_{\exp \theta Y} P_{\theta Y}-\Delta_{f_{0}} Y\right| & =\left|\int_{0}^{1} \frac{d}{d s}\left[P_{-s \theta Y} \Delta_{\exp s \theta Y} P_{s \theta Y} Y\right] d s\right| \\
& \leq \int_{0}^{1}\left|\frac{d}{d s}\left[P_{-s \theta Y} \Delta_{\exp s \theta Y} P_{s \theta Y} Y\right]\right| d s .
\end{aligned}
$$

According to (7.11) and (cf. [6], 12.7) $\nabla_{Z}^{u} \nabla_{c^{\prime}} V=\nabla_{c^{\prime}} \nabla_{Z}^{u}+\sum_{i=1}^{u} \nabla_{Z}^{u-i} R\left(V, c^{\prime}\right) \nabla_{Z}^{i-1} V$, and according to (7.11),

$$
\left.\frac{d}{d s}\left[P_{-s \theta Y}\left(\Delta_{\exp s \theta Y} P_{s \theta Y} Y\right)\right]=P_{-s \theta Y} \nabla_{c^{\prime}} \Delta_{\exp s \theta Y} P_{s \theta Y} Y\right)
$$


We estimate

$$
\begin{aligned}
\left.\mid P_{-s \theta Y} \nabla_{c^{\prime}} \Delta_{\exp s \theta Y} P_{s \theta Y} Y\right) \mid= & \left.\mid \nabla_{c^{\prime}} \Delta_{\exp s \theta Y} P_{s \theta Y} Y\right) \mid \\
= & \left.\left|g_{0}^{i j} \nabla_{c^{\prime}} \nabla_{j} \nabla_{i}\right|_{\exp s \theta Y} P_{s \theta Y} Y\right) \mid \\
= & \left.\left|g_{0}^{i j} \nabla_{j} \nabla_{c^{\prime}} \nabla_{i}\right|_{\exp s \theta Y} P_{s \theta Y} Y\right) \mid \\
& \left.+\left.g_{0}^{i j} R\left(c^{\prime},(d(\exp s \theta Y))\left(e_{j}\right)\right) \nabla_{i}\right|_{\exp s \theta Y} P_{s \theta Y} Y\right) \mid \\
= & \left.\mid g_{0}^{i j} \nabla_{j} \nabla_{i} \nabla_{c^{\prime}} P_{s \theta Y} Y\right) \\
& +g_{0}^{i j} \nabla_{j} R\left(c^{\prime},(d(\exp s \theta Y))\left(e_{i}\right)\right) P_{s \theta Y} Y \\
& \left.+\left.g_{0}^{i j} R\left(c^{\prime},(d(\exp s \theta Y))\left(e_{j}\right)\right) \nabla_{i}\right|_{\exp s \theta Y} P_{s \theta Y} Y\right) \mid \\
= & \mid g_{0}^{i j} \nabla_{j} R\left(c^{\prime},(d(\exp \theta Y))\left(e_{i}\right) P_{s \theta Y} Y\right. \\
& \left.+\left.g_{0}^{i j} R\left(c^{\prime},(d(\exp s \theta Y))\left(e_{j}\right)\right) \nabla_{i}\right|_{\exp s \theta Y} P_{s \theta Y} Y\right) \mid \\
\leq & c_{1}\left[\left|\nabla c^{\prime}\right||Y|+\left|c^{\prime}\right| p_{1}(|Y|,|\nabla Y|)|Y|\right. \\
& \left.+\left|c^{\prime}\right|\left|\nabla\left(P_{s \theta Y} Y\right)\right|+\left|c^{\prime}\right|\left|\nabla\left(P_{s \theta Y} Y\right)\right|\right] \\
\leq & c_{1}\left[\left|\nabla\left(P_{s \theta Y} Y\right)\right||Y|+|Y| p_{1}(|Y|,|\nabla Y|)|Y|\right. \\
& \left.+|Y|\left|\nabla\left(P_{s \theta Y} Y\right)\right|+|Y|\left|\nabla\left(P_{s \theta Y} Y\right)\right|\right] \\
= & C\left[|Y|\left|\nabla\left(P_{s \theta Y} Y\right)\right|+p_{1}(|Y|,|\nabla Y|)|Y|^{2}\right] .
\end{aligned}
$$

Here we used

$$
\begin{gathered}
\left|c^{\prime}\right|=\left|P_{s \theta Y} \theta Y\right|=|\theta Y| \leq|Y|, \\
\mid \nabla^{i}\left(d(\exp s Y)\left|\leq p_{i}\left(|Y|,|\nabla Y|, \cdots,\left|\nabla^{i} Y\right|\right), \quad\right|(d(\exp \theta Y))\left(e_{i}\right) \mid \leq c_{1},\right.
\end{gathered}
$$

where $p_{i}$ is a polynomial in the indicated variables with positive coefficients depending on $r_{i n j}$, curvature and on bounds for $\left|\nabla^{j} d f_{t_{0}}\right|$ (cf. [12], p.362). But according to (4.10), we have such bounds independent of $t_{0}$. Further we used $\left|\nabla_{X} Y\right| \leq|X||\nabla Y|$, $|\nabla| Y|| \leq|\nabla Y|$ under our curvature assumptions.

We must estimate

$$
\nabla\left(P_{s \theta Y} Y\right)=\left(\nabla P_{s \theta Y}\right) Y+P_{s \theta Y}(\nabla Y)
$$

which amounts to the estimate of

$$
\nabla P_{s \theta Y}
$$

Here we consider $\nabla P_{s \theta Y}$ as an isomorphism $f_{t_{0}}^{*} T M \rightarrow(\operatorname{exps} \theta Y)^{*} T M, \nabla P_{s \theta Y} \equiv$ $\left(f_{t_{0}}^{*} \nabla\right) P_{s \theta Y} \cdot \nabla \equiv f_{t_{0}}^{*} \nabla$ is independent of $s$. Hence, in (7.14), (7.15) $\frac{d}{d s}$ and $\nabla$ are exchangeable.

We get with $X(s)=P_{s \theta Y} Y$ that

$$
\begin{aligned}
\frac{d}{d s}\left(\nabla P_{s \theta Y}\right) Y= & {\left[\left(\nabla \frac{d}{d s} P_{s \theta Y}\right)\right] } \\
= & \nabla\left(\frac{d}{d s} P_{s \theta Y}\right) Y-\frac{d}{d s} P_{s \theta Y} \nabla Y \\
= & -\nabla\left\langle c^{\prime}, \Gamma^{o p} P_{s \theta Y} Y\right\rangle-\left(-\left\langle c^{\prime}, \Gamma^{o p} P_{s \theta Y} \nabla Y\right\rangle\right) \\
= & -\left[\left\langle\nabla c^{\prime}, \Gamma^{o p} P_{s \theta Y} Y\right\rangle+\left\langle c^{\prime},\left(\nabla \Gamma^{o p}\right) P_{s \theta Y} Y\right\rangle\right. \\
& +\left\langle c^{\prime}, \Gamma^{o p}\left(\nabla P_{s \theta Y}\right) Y\right\rangle+\left\langle c^{\prime}, \Gamma^{o p}\left(P_{s \theta Y}\right) \nabla Y\right\rangle-\left\langle c^{\prime}, \Gamma^{o p}\left(P_{s \theta Y}\right) \nabla Y\right\rangle \\
= & -\left[\left\langle\nabla c^{\prime}, \Gamma^{o p} P_{s \theta Y} Y\right\rangle+\left\langle c^{\prime},\left(\nabla \Gamma^{o p}\right) P_{s \theta Y} Y\right\rangle\right. \\
& \left.+\left\langle c^{\prime}, \Gamma^{o p}\left(\nabla P_{s \theta Y}\right) Y\right\rangle\right] .
\end{aligned}
$$


We see that (7.23) is a linear ODE for the morphism $\nabla P_{s \theta Y}$ :

$$
\begin{aligned}
\frac{d}{d s}\left(\nabla P_{s \theta Y}\right)(\cdot)= & -\left[\left\langle\nabla c^{\prime}, \Gamma^{o p} P_{s \theta Y}(\cdot)\right\rangle+\left\langle c^{\prime},\left(\nabla \Gamma^{o p}\right) P_{s \theta Y}(\cdot)\right\rangle\right. \\
& +\left\langle c^{\prime}, \Gamma^{o p}\left(\nabla P_{s \theta Y}\right)(\cdot)\right\rangle,
\end{aligned}
$$

that is,

$$
\frac{d}{d s}\left(\nabla P_{s \theta Y}\right)=f\left(\nabla P_{s \theta Y}, c^{\prime}, \nabla c^{\prime}, \Gamma^{o p}, \nabla \Gamma^{o p}, P_{s \theta Y}\right),
$$

and $f$ is continuous, bounded and satisfies a Lipschitz condition, if $c^{\prime}, \nabla c^{\prime}, \Gamma^{o p}, \nabla \Gamma^{o p}$, $P_{s \theta Y}$ are bounded. The latter is the case. Hence $\nabla P_{s \theta Y}$ itself is bounded on $M$ :

$$
\left|\nabla P_{s \theta Y}\right| \leq C_{1}^{\prime} .
$$

Completely parallel we obtain from differentiation of (7.21), (7.24) and (7.25)

$$
\left|\nabla^{2} P_{s \theta Y}\right| \leq C_{2}^{\prime}
$$

For higher derivatives of $P_{s \theta Y}$, we cannot apply the classical existence theorem and its conclusions. We assumed $r>3$ and inferred $\dot{c}$ is $C^{2}$. But we can only conclude $\left|\nabla^{i} c^{\prime}\right| \in L_{2}, i \geq 3$. In these cases we apply Theorem 26.1 from [28], p.384 and can still conclude

$$
\left|\nabla^{i} P_{s \theta Y}\right| \in L_{2}, \quad r \geq i \geq 2 .
$$

We obtain from (7.19),(7.25),(7.26), and (7.27)

$$
\left|P_{-s \theta Y} \nabla_{c^{\prime}}\left(\Delta_{\exp s \theta Y} P_{s \theta Y} Y\right)\right| \leq p_{0}(|Y|,|\nabla Y|),
$$

where $p_{0}$ is a polynomial in the indicated variables with pointwise coefficients (one of them is a Sobolev function) and without monomials of degree not greater than 1 . Since all monomials stem from the Leibniz rule, we can infer that they are in $L_{2}$, and we obtain

$$
\begin{aligned}
\left|P_{-\theta Y} \Delta_{\exp s \theta} P_{\theta Y}-\Delta_{f_{t_{0}}} Y\right|_{L_{2}} & \leq C\left(|Y|_{L_{2}}+|\nabla Y|_{L_{2}}\right)|Y|_{L_{2}} \\
& \leq C|Y|_{r}|Y|_{r} .
\end{aligned}
$$

Next we have to estimate

$$
\nabla^{u} P_{-s \theta Y} \nabla_{c^{\prime}} \Delta_{\exp s \theta Y} P_{\theta Y} Y
$$

which reduces to the estimate of $\nabla^{i} P_{\theta Y}$ and

$$
\nabla^{j} \nabla_{c^{\prime}} \Delta_{\exp s \theta Y} P_{\theta Y} Y .
$$

We exchange the differentiations in (7.29), a procedure which we use in the sequel. To estimate $\nabla^{u} V$ for a vector field $V$, we have to estimate expansions

$$
\nabla_{Z_{u}} \cdots \nabla_{Z_{1}}
$$

Since $\nabla_{X+Y} \nabla_{X-Y}=\nabla_{X}^{2}-\nabla_{Y}^{2}+\nabla_{Y} \nabla_{X}-\nabla_{X} \nabla_{Y}$ and we control the curvature, we would be done controling $\nabla_{Z}^{u} V=\nabla_{Z} \cdots \nabla_{Z} V$. 
According to (7.18),

$$
\begin{aligned}
& \left|\nabla^{u} g_{0}^{i j} \nabla_{c^{\prime}} \nabla_{j} \nabla_{i}\right|_{\exp s \theta Y} P_{s \theta Y} Y \mid \\
= & \mid g_{0}^{i j} \nabla^{u}\left[\nabla_{j} R\left(c^{\prime},(d(\exp s \theta Y))\left(e_{i}\right)\right) P_{s \theta Y} Y\right. \\
& \left.+\left.R\left(c^{\prime},(d(\exp s \theta Y))\left(e_{j}\right)\right) \nabla_{i}\right|_{\exp s \theta Y} P_{s \theta Y} Y\right] \mid \\
\leq & C\left\{\sum_{i_{1}+i_{2}+i_{3}=u}\left|\nabla^{i_{1}} c^{\prime}\right|\left|\nabla^{i_{2}} d(\exp s \theta Y)\right|\left|\nabla^{i_{3}} P_{s \theta Y}\right|\right. \\
& \left.+\sum_{j_{1}+j_{2}+j_{3}=u}\left|\nabla^{j_{1}} c^{\prime}\right|\left|\nabla^{j_{2}} d(\exp s \theta Y)\right|\left|\nabla^{j_{3}+1} P_{s \theta Y}\right|\right\} .
\end{aligned}
$$

Now we estimate the single terms of (7.30) according to (7.20), (7.27) and obtain easily

$$
\left|\nabla^{u} g_{0}^{i j} \nabla_{c^{\prime}} \nabla_{j} \nabla_{i}\right|_{\exp s \theta Y} P_{s \theta Y} Y \mid \leq P_{u}\left(|Y|,|\nabla Y|, \cdots,\left|\nabla^{u-1} Y\right|\right),
$$

where $P_{u}$ is a polynomial in the indicated variables with positive coefficients (some of them are Sobolev functions) independent of $t_{0}$ and without monomial of degree not greater that one. Since all monomials stem from the Leibniz rule, we can apply the module structure theorem and infer that they are in $L_{2}$ and we obtain for $u \leq r-2$

$$
\begin{gathered}
\left|\nabla^{u} P_{s \theta Y} g_{0}^{i j} \nabla_{c^{\prime}} \nabla_{j} \nabla_{i} P_{s \theta Y} Y\right|_{L_{2}} \leq \widetilde{Q}_{u}\left(|Y|_{r}\right), \\
\left|P_{s \theta Y} g_{0}^{i j} \nabla_{c^{\prime}} \nabla_{j} \nabla_{i} P_{s \theta Y} Y\right|_{r-2}=Q_{r-2}\left(|Y|_{r}\right)=P_{r-2}\left(|Y|_{r}\right)|Y|_{r},
\end{gathered}
$$

where $P_{r-2}\left(|Y|_{r}\right):=Q_{r-2}\left(|Y|_{r}\right) /|Y|_{r}$ is a polynomial in the indicated variables with positive coeffcients independent of $t_{0}$ and without constant term, in particular

$$
\left|P_{-\theta Y} \Delta_{\exp \theta Y} P_{\theta Y} Y-\Delta_{f_{t_{0}}} Y\right|_{r-2} \leq o(1)|Y|_{r},
$$

where $o(1) \rightarrow 0\left(|Y|_{r} \rightarrow 0\right)$ with rate independent of $t_{0}$.

There remains to estimate

$$
t r_{g_{0}} R^{g_{t_{0}}}\left(Y, d f_{t_{0}}\right) d f_{t_{0}}-P_{-\theta Y} t r_{g_{0}} R^{g_{0}}\left(P_{\theta Y} Y, d(\exp \theta Y)\right) d(\exp \theta Y)
$$

and

$$
P_{-\theta Y} \operatorname{tr}_{g_{0}} \nabla_{c^{\prime}}\left(\nabla^{g_{t}}-\nabla^{g_{0}}\right) d(\exp \theta Y) .
$$

We write (7.34) as

$$
\begin{aligned}
& \operatorname{tr}_{g_{0}} R^{g_{t_{0}}}\left(Y, d f_{t_{0}}\right) d f_{t_{0}}-P_{-\theta Y} \operatorname{tr}_{g_{0}} R^{g_{t_{0}}}\left(P_{\theta Y} Y, d(\exp \theta Y)\right) d(\exp \theta Y) \\
& +P_{-\theta Y} \operatorname{tr}_{g_{0}} R^{g_{t_{0}}}\left(P_{\theta Y} Y, d(\exp \theta Y)\right) d(\exp \theta Y) \\
& -P_{-\theta Y} \operatorname{tr}_{g_{0}} R^{g_{0}}\left(P_{\theta Y} Y, d(\exp \theta Y)\right) d(\exp \theta Y) .
\end{aligned}
$$

In normal coordinates,

$$
\begin{aligned}
(7.36) & =-\int_{0}^{1} \frac{d}{d s}\left[P_{-s \theta Y} \operatorname{tr}_{g_{0}} R^{g_{t_{0}}}\left(P_{s \theta Y} Y, d(\exp s \theta Y)\right) d(\exp s \theta Y)\right] d s \\
& \left.=-\int_{0}^{1} P_{-s \theta Y} \nabla_{c^{\prime}} t r_{g_{0}} R^{g_{t_{0}}}\left(P_{s \theta Y} Y, d(\exp s \theta Y)\right) d(\exp s \theta Y)\right] d s .
\end{aligned}
$$


But

$$
\left|P_{-s \theta Y} \nabla_{c^{\prime}} t_{g_{0}} R^{g_{t_{0}}}\left(P_{s \theta Y} Y, d(\exp s \theta Y)\right) d(\exp s \theta Y)\right| \leq C|Y||\nabla Y|,
$$

we obtain (as always, by the module structure theorem)

$$
|(7.36)|_{L_{2}} \leq C|Y|_{L_{2}}|\nabla Y|_{L_{2}} \leq C|Y|_{r}|Y|_{r}=P_{0}\left(|Y|_{r}\right)|Y|_{r} .
$$

We recall our standard formula

$$
\nabla_{Z}^{u} \nabla_{c^{\prime}} V=\nabla_{c^{\prime}} \nabla_{Z}^{u}+\sum_{i=1}^{u} \nabla_{Z}^{u-i} R\left(V, c^{\prime}\right) \nabla_{Z}^{i-1} V
$$

Applying this to (7.29) yields

$$
\begin{aligned}
& \left|\nabla_{Z}^{u} \nabla_{c^{\prime}} g_{0}^{i j} R^{g_{t_{0}}}\left(P_{s \theta Y},(d(\exp s \theta Y))\left(e_{i}\right)\right)(d(\exp s \theta Y))\left(e_{j}\right)\right| \\
= & \mid \nabla_{c^{\prime}} \nabla_{Z}^{u} g_{0}^{i j} R^{g_{t_{0}}}\left(P_{s \theta Y},(d(\exp s \theta Y))\left(e_{i}\right)\right)(d(\exp s \theta Y))\left(e_{j}\right) \\
& \left.+\sum_{i=1}^{u} \nabla_{Z}^{u-i} R\left(R^{g_{t_{0}}}\left(P_{s \theta Y},(d(\exp s \theta Y))\left(e_{i}\right)\right)(d(\exp s \theta Y))\left(e_{j}\right)\right), c^{\prime}\right) \\
& \nabla_{Z}^{i-1} R^{g_{t_{0}}}\left(P_{s \theta Y},(d(\exp s \theta Y))\left(e_{i}\right)\right)(d(\exp s \theta Y))\left(e_{j}\right) \mid \\
\leq & C\left\{\left|c^{\prime}\right|\left[\sum_{i_{1}+i_{2}+i_{3}+i_{4}=u+1}\left|\nabla^{i_{1}} R^{g_{t_{0}}}\right|\left|\nabla^{i_{2}} P_{s \theta Y} Y\right| p_{i_{3}}\left(|Y|, \cdots,\left|\nabla^{i_{3}} Y\right|\right) p_{i_{4}}\left(|Y|, \cdots,\left|\nabla^{i_{4}} Y\right|\right)\right]\right. \\
& +\sum_{i=1}^{u} \sum_{i_{1}+\cdots+i_{5}=u}\left|\nabla^{i_{1}} R^{g_{t_{0}}}\right|\left|\nabla^{i_{2}} P_{s \theta Y} Y\right| p_{i_{3}}\left(|Y|, \cdots,\left|\nabla^{i_{3}} Y\right|\right) p_{i_{4}}\left(|Y|, \cdots,\left|\nabla^{i_{4}} Y\right|\right)\left|\nabla^{i_{5}} c^{\prime}\right| \\
& \left.\cdot \sum_{j_{1}+j_{2}+j_{3}+j_{4}=u+1}\left|\nabla^{j_{1}} R^{g_{t_{0}}}\right|\left|\nabla^{j_{2}} P_{s \theta Y} Y\right| p_{j_{3}}\left(|Y|, \cdots,\left|\nabla^{j_{3}} Y\right|\right) p_{j_{4}}\left(|Y|, \cdots,\left|\nabla^{j_{4}} Y\right|\right)\right\} \\
= & \tilde{P}_{u}\left(|Y|, \cdots,\left|\nabla^{u+1} Y\right|\right),
\end{aligned}
$$

where $P_{u}$ is a polynomial in the indicated variables with positive coefficients (possibly Sobolev functions) independent of $t_{0}$ and without monomials of degree not greater that one. Hence

$$
\begin{aligned}
& \left.\left.\mid \nabla^{u} P_{s \theta Y} \nabla_{c^{\prime}} g_{0}^{i j} R^{g_{t_{0}}}\left(P_{s \theta Y},(d(\exp s \theta Y))\right)\left(e_{i}\right)\right)(d(\exp s \theta Y))\right)\left.\left(e_{j}\right)\right|_{L_{2}} \\
& \leq \sum_{i_{1}+i_{2}=u} \| \nabla^{i_{1}} P_{s \theta Y}\left|\tilde{p}_{i_{2}+1}\left(|Y|, \cdots,\left|\nabla^{i_{2}+1} Y\right|\right)\right|_{L_{2}} \\
& \leq \tilde{Q}_{u}\left(|Y|_{r}\right) \\
& \left.\mid \operatorname{tr}_{g_{0}} R^{g_{t_{0}}}\left(Y, d f_{t_{0}}\right) d f_{t_{0}}-P_{-\theta Y} R^{g_{t_{0}}}\left(P_{\theta Y} Y,(d(\exp \theta Y))\right) d(\exp \theta Y)\right)\left.\right|_{r-2} \\
& \leq Q_{r-2}\left(|Y|_{r}\right) \\
& =P_{r-2}\left(|Y|_{r}\right)|Y|_{r}
\end{aligned}
$$

thus

$$
\left.\mid \operatorname{tr}_{g_{0}} R^{g_{t_{0}}}\left(Y, d f_{t_{0}}\right) d f_{t_{0}}-P_{-\theta Y} R^{g_{t_{0}}}\left(P_{\theta Y} Y,(d(\exp \theta Y))\right) d(\exp \theta Y)\right)\left.\right|_{r-2} \leq o(1)|Y|_{r}
$$


$o(1) \rightarrow 0(|Y| \rightarrow 0)$ with rate independent of $t_{0},(7.36)$ is done.

We write (7.37) in normal coordinates(w.r.t. $g_{0}$ ) as

$$
\begin{aligned}
(7.37)= & \int_{0}^{1} \frac{d}{d s}\left[P_{-s \theta Y} \operatorname{tr}_{g_{0}} R^{g_{s t_{0}}}\left(P_{-s \theta Y} Y, d(\exp s \theta Y)\right) d(\exp s \theta Y)\right. \\
& \left.-P_{-s \theta Y} \operatorname{tr}_{g_{0}} R^{g_{t_{0}}}\left(P_{-s \theta Y} Y, d(\exp s \theta Y)\right) d(\exp s \theta Y)\right] d s \\
= & \int_{0}^{1} P_{-s \theta Y} \nabla_{c^{\prime}}\left[\operatorname{tr}_{g_{0}} R^{g_{s t_{0}}}\left(P_{-s \theta Y} Y, d(\exp s \theta Y)\right) d(\exp s \theta Y)\right. \\
& \left.-\operatorname{tr}_{g_{0}} R^{g_{t_{0}}}\left(P_{-s \theta Y} Y, d(\exp s \theta Y)\right) d(\exp s \theta Y)\right] d s .
\end{aligned}
$$

But both terms of $\left|P_{-s \theta Y} \nabla_{c^{\prime}}[\cdots]\right|$ can in the same manner be estimated as (7.41)(7.44), then

$$
|(7.37)|_{r-2} \leq p_{r-1}\left(|Y|_{r}\right)|Y|_{r} .
$$

From (7.44) and (7.45) it follows that

$$
|(7.34)|_{r-2} \leq P_{r-1}\left(|Y|_{r}\right)|Y|_{r}=o(1)|Y|_{r},
$$

where $P_{r-1}$ without constant term and with positive coefficients independent of $t_{0}$.

We finally estimate

$$
\begin{gathered}
P_{-\theta Y} \operatorname{tr}_{g_{0}} \nabla_{c^{\prime}}\left(\nabla^{g_{t}}-\nabla^{g_{0}}\right) d(\exp \theta Y) \\
=\int_{0}^{1} \frac{d}{d s}\left[P_{-s \theta Y} \operatorname{tr}_{g_{0}} \nabla_{c^{\prime}}\left(\nabla^{g_{s t}}-\nabla^{g_{0}}\right) d(\exp s \theta Y)\right] d s \\
=\int_{0}^{1} P_{-s \theta Y} \nabla_{c^{\prime}} t_{g_{0}} \nabla_{c^{\prime}}\left(\nabla^{g_{s t}}-\nabla^{g_{0}}\right) d(\exp s \theta Y) d s \\
\left|\nabla^{u} \nabla_{c^{\prime}} \nabla_{c^{\prime}} t r_{g_{0}}\left(\nabla^{g_{s t}}-\nabla^{g_{0}}\right) d(\exp s \theta Y)\right| \\
=\mid t r_{g_{0}}\left[\nabla_{c^{\prime}} \nabla^{u} \nabla_{c^{\prime}}\left(\nabla^{g_{s t}}-\nabla^{g_{0}}\right) d(\exp s \theta Y)\right. \\
+\sum_{i=1}^{u} \nabla^{u-i} R\left(\nabla_{c^{\prime}}\left(\nabla^{g_{s t}}-\nabla^{g_{0}}\right)\left(d(\exp s \theta Y), c^{\prime}\right) \nabla^{i-1} \nabla_{c^{\prime}}\left(\nabla^{g_{s t}}-\nabla^{g_{0}}\right) d(\exp s \theta Y) \mid\right. \\
=\mid t r_{g_{0}}\left[\nabla_{c^{\prime}} \nabla_{c^{\prime}} \nabla^{u}\left(\nabla^{g_{s t}}-\nabla^{g_{0}}\right) d(\exp s \theta Y)\right. \\
+\sum_{i=1}^{u} \nabla_{c^{\prime}} \nabla^{u-i} R\left(\left(\nabla^{g_{s t}}-\nabla^{g_{0}}\right)\left(d(\exp s \theta Y), c^{\prime}\right) \nabla^{i-1}\left(\nabla^{g_{s t}}-\nabla^{g_{0}}\right) d(\exp s \theta) \mid\right. \\
+\sum_{i=1}^{u} \nabla^{u-i} R\left(\nabla_{c^{\prime}}\left(\nabla^{g_{s t}}-\nabla^{g_{0}}\right)\left(d(\exp s \theta Y), c^{\prime}\right) \nabla^{i-1} \nabla_{c^{\prime}}\left(\nabla^{g_{s t}}-\nabla^{g_{0}}\right) d(\exp s \theta Y)\right] \mid \\
\leq C\left[\left|c^{\prime}\right|^{2} \sum_{i_{1}+i_{2}=u+2}\left|\nabla^{i_{1}}\left(\nabla^{g_{s t}}-\nabla^{g_{0}}\right)\right|\left|\nabla^{i_{2}} d(\exp s \theta Y)\right|\right. \\
+\left|\nabla^{\prime}\right| \sum_{i=1}^{u+1} \sum_{i_{1}+\cdots+i_{4}=u+1-i}^{i_{4}+i-1}\left(\nabla^{g_{s t}}-\nabla^{g_{0}}\right) d(\exp s \theta Y) \mid \\
\left|\nabla^{i_{1}} \nabla_{c^{\prime}}\left(\nabla^{g_{s t}}-\nabla^{g_{0}}\right)\right|\left|\nabla^{i_{2}} d(\exp s \theta Y)\right|\left|\nabla^{i_{3}} c^{\prime}\right|
\end{gathered}
$$




$$
\begin{aligned}
& +\sum_{i=1}^{u} \sum_{i_{1}+\cdots+i_{4}=u-i}\left|\nabla^{i_{1}} \nabla_{c^{\prime}}\left(\nabla^{g_{s t}}-\nabla^{g_{0}}\right)\right|\left|\nabla^{i_{2}} d(\exp s \theta Y)\right|\left|\nabla^{i_{3}} c^{\prime}\right| \\
& \cdot\left|\nabla^{i_{4}+i-1} \nabla_{c^{\prime}}\left(\nabla^{g_{s t}}-\nabla^{g_{0}}\right) d(\exp s \theta Y)\right| .
\end{aligned}
$$

We estimate the single terms.

$$
\begin{gathered}
\left|c^{\prime}\right|^{2} \leq 2\left(\left|Y_{1}\right|+\left|Y_{2}-Y_{1}\right|\right)^{2}, \\
\left|\nabla^{i_{1}}\left(\nabla^{g_{s t}}-\nabla^{g_{0}}\right)\right| \leq\left\{\begin{array}{cc}
\sup _{x}\left|\nabla^{i_{1}}\left(\Gamma\left(g_{s t}\right)-\Gamma\left(g_{0}\right)\right)\right|<\infty, & 0 \leq i_{1} \leq 1, \\
\text { Sobolev function, } & i_{1} \geq 2 .
\end{array}\right. \\
\left|\nabla^{i_{2}}(d(\exp s \theta Y))\left(e_{i}\right)\right| \leq\left\{\begin{array}{cc}
P_{i_{2}}\left(|Y|, \cdots,\left|\nabla^{i_{2}} Y\right|\right), & i_{2} \geq 1, \\
\text { const. }, & i_{2}=0 .
\end{array}\right. \\
\left|\nabla^{i_{3}} c^{\prime}\right|=\left|\nabla^{i_{3}}\left(P_{s \theta Y} Y\right)\right| \leq \sum_{j_{1}+j_{2}=i_{3}}\left|\nabla^{j_{1}} P_{s \theta Y}\right|\left|\nabla^{j_{2}} Y\right| .
\end{gathered}
$$

We have in $(7.48),(7.49)$ terms of the kind $\nabla^{j} \nabla_{c^{\prime}}\left(\nabla^{g_{s t}}-\nabla^{g_{0}}\right) d(\exp s \theta Y)$, but

$$
\begin{aligned}
& \nabla^{j} \nabla_{c^{\prime}}\left(\nabla^{g_{s t}}-\nabla^{g_{0}}\right) d(\exp s \theta Y)=\nabla_{c^{\prime}} \nabla^{j}\left(\nabla^{g_{s t}}-\nabla^{g_{0}}\right) d(\exp s \theta Y) \\
& +\sum_{i=1}^{j} \nabla^{j-i} R\left(\left(\nabla^{g_{s t}}-\nabla^{g_{0}}\right) d(\exp s \theta Y), c^{\prime}\right) \nabla^{i-1}\left(\nabla^{g_{s t}}-\nabla^{g_{0}}\right) d(\exp s \theta Y),
\end{aligned}
$$

hence,

$$
\begin{aligned}
& \left|\nabla^{j} \nabla_{c^{\prime}}\left(\nabla^{g_{s t}}-\nabla^{g_{0}}\right) d(\exp s \theta Y)\right| \\
\leq & \left|c^{\prime}\right| \sum_{j_{1}+j_{2}=j}\left|\nabla^{j_{1}}\left(\nabla^{g_{s t}}-\nabla^{g_{0}}\right)\right|\left|\nabla^{j_{2}} d(\exp s \theta Y)\right| \\
& +\sum_{i=1}^{j} \sum_{j_{1}+\cdots+j_{4}=j-i}\left|\nabla^{j_{1}}\left(\nabla^{g_{s t}}-\nabla^{g_{0}}\right)\right|\left|\nabla^{j_{2}} d(\exp s \theta Y)\right|\left|\nabla^{j_{3}} c^{\prime}\right| \\
& \cdot \sum_{k_{1}+k_{2}=j_{4}+i-1}\left|\nabla^{k_{1}}\left(\nabla^{g_{s t}}-\nabla^{g_{0}}\right)\right|\left|\nabla^{k_{2}} d(\exp s \theta Y)\right| .
\end{aligned}
$$

Inserting (7.50)-(7.53) into (7.47)-(7.49) yields

$$
\left|\nabla^{u} \nabla_{c^{\prime}} \nabla_{c^{\prime}} t r_{g_{0}}\left(\nabla^{g_{s t}}-\nabla^{g_{0}}\right) d(\exp s \theta Y)\right| \leq \tilde{Q}_{u}\left(|Y|, \cdots,\left|\nabla^{u+2} Y\right|\right),
$$

where $\tilde{Q}_{u}$ is a polynomial in the indicated variables with positive coefficients (possibly Sobolev functions) independent of $t_{0}$ and without monomials of degree not greater that one.

We conclude that

$$
\begin{aligned}
\left|\nabla^{u} P_{-s \theta Y} \operatorname{tr}_{g_{0}} \nabla_{c^{\prime}}\left(\nabla^{g_{s t}}-\nabla^{g_{0}}\right) d(\exp s \theta Y)\right| & \leq \sum_{i_{1}+i_{2}=u}\left|\nabla^{i_{1}} P_{-s \theta Y}\right| \tilde{Q}_{i_{2}}\left(|Y|, \cdots,\left|\nabla^{i_{2}+2} Y\right|\right) \\
& =Q_{u}\left(|Y|, \cdots,\left|\nabla^{u+2} Y\right|\right) .
\end{aligned}
$$


All monomials in (7.52) stem from the Leibniz rule, and we can apply the module structure theorem. Summing up $u=0, \cdots, r-2$, we get

$$
\begin{aligned}
\left|P_{-s \theta Y} t r_{g_{0}} \nabla_{c^{\prime}}\left(\nabla^{g_{s t}}-\nabla^{g_{0}}\right) d(\exp s \theta Y)\right|_{r-2} & \leq Q_{r-2}\left(|Y|_{r}\right) \\
& =P_{r-2}\left(|Y|_{r}\right)|Y|_{r} \\
& =o(1)|Y|_{r},
\end{aligned}
$$

where $o(1) \rightarrow 0$ as $|Y|_{r} \rightarrow 0$, and $p_{r-2}\left(|Y|_{r}\right)$ is a polynomial in the indicated variables with positive coefficients independent of $t_{0}$ and without constant term.

Finally, we infer from (7.33), (7.46) and (7.55) that

$$
|g(t, Y)-g(t, 0)|_{r-2} \leq o(1)|Y|_{r},
$$

where $o(1) \rightarrow 0\left(|Y|_{r} \rightarrow 0\right)$ with rate independent of $t_{0}$. The proof of Proposition 7.4 is done.

Proposition 7.5. Suppose $f_{t_{0}}:\left(M^{2}, g_{0}\right) \rightarrow\left(M^{2}, g_{t_{0}}\right)$ is a harmonic diffeomorphism. Then there holds for $Y_{1}, Y_{2} \in \Omega^{2, r}\left(f_{t_{0}}^{*} T M\right), r>3,\left|Y_{i}\right|<\min \left\{\frac{1}{2}, \inf _{t} r_{i n j}\left(g_{t}\right)\right\}$, $\left|Y_{i}\right|_{r}<\frac{1}{2}$

$$
\left|g\left(t, Y_{1}\right)-g\left(t, Y_{2}\right)\right|_{r-2} \leq o_{2}(1)\left|Y_{1}-Y_{2}\right|_{r}
$$

where

$$
o_{2}(1) \rightarrow 0 \quad \text { as } \quad\left|Y_{i}\right|_{r} \rightarrow 0
$$

with rate independent of $t_{0}$.

Proof.

$$
\begin{aligned}
& \left|g\left(t, Y_{1}\right)-g\left(t, Y_{2}\right)\right|_{r-2} \\
= & \left|g\left(t, Y_{1}\right)-g\left(t, Y_{1}+\left(Y_{2}-Y_{1}\right)\right)\right|_{r-2} \\
\leq & \left.\sup _{0<\theta<1}\left|\frac{d}{d \tau}\right|_{\tau=0} g\left(t, Y_{1}+(\theta+\tau)\left(Y_{2}-Y_{1}\right)\right)\right|_{r-2} \\
= & \sup _{0<\theta<1}\left|\frac{d}{d \tau}\right|_{\tau=0} F_{X}\left(t_{0}, 0\right)\left(Y_{1}+(\theta+\tau)\left(Y_{2}-Y_{1}\right)\right) \\
& -\left.\left.\frac{d}{d \tau}\right|_{\tau=0}\left[P_{-\left(Y_{1}+(\theta+\tau)\left(Y_{2}-Y_{1}\right)\right)} \operatorname{tr}_{g_{0}} \nabla^{g_{t}} d\left(\exp \left(Y_{1}+(\theta+\tau)\left(Y_{2}-Y_{1}\right)\right)\right)\right]\right|_{r-2} \\
= & \sup _{0<\theta<1} \mid F_{X}\left(t_{0}, 0\right)\left(Y_{2}-Y_{1}\right) \\
& -\left.\left.\frac{d}{d \tau}\right|_{\tau=0}\left[P_{-\left(Y_{1}+(\theta+\tau)\left(Y_{2}-Y_{1}\right)\right)} \operatorname{tr}_{g_{0}} \nabla^{g_{t}} d\left(\exp \left(Y_{1}+(\theta+\tau)\left(Y_{2}-Y_{1}\right)\right)\right)\right]\right|_{r-2} \\
= & \sup _{0<\theta<1} \mid-\Delta_{f_{t_{0}}}\left(Y_{2}-Y_{1}\right)+t r_{g_{0}} R^{g_{t_{0}}}\left(Y_{2}-Y_{1}, d f_{t_{0}}\right) d f_{t_{0}} \\
& -\left.\left.\frac{d}{d \tau}\right|_{\tau=0}\left[P_{-\left(Y_{1}+(\theta+\tau)\left(Y_{2}-Y_{1}\right)\right)} \operatorname{tr}_{g_{0}} \nabla^{g_{t}} d\left(\exp \left(Y_{1}+(\theta+\tau)\left(Y_{2}-Y_{1}\right)\right)\right)\right]\right|_{r-2}
\end{aligned}
$$

We calculate

$$
\left.\frac{d}{d \tau}\right|_{\tau=0}\left[P_{-\left(Y_{1}+(\theta+\tau)\left(Y_{2}-Y_{1}\right)\right)} \operatorname{tr}_{g_{0}} \nabla^{g_{t}} d\left(\exp \left(Y_{1}+(\theta+\tau)\left(Y_{2}-Y_{1}\right)\right)\right)\right]
$$


as follows. Denote $Y_{\theta}:=Y_{1}+\theta\left(Y_{2}-Y_{1}\right)$, let $P_{Y_{\theta}}$ be the parallel transport along $\exp _{f_{t_{0}}} s Y_{\theta}$ from $f_{t_{0}}$ to $\exp Y_{\theta}$, and $P_{-Y_{\theta}}$ be the corresponding parallel transport from $\exp Y_{\theta}$ to $f_{t_{0}}$. Denote by $c_{Y_{\theta}}(\tau)$ the curve $\exp \left(Y_{\theta}+\tau\left(Y_{2}-Y_{1}\right)\right)$, and let $P_{0 \rightarrow \tau}$ be the parallel transport along $c_{Y_{\theta}}(\tau)$ from $\exp Y_{\theta}$ to $\exp \left(Y_{\theta}+\tau\left(Y_{2}-Y_{1}\right)\right), P_{\tau \rightarrow 0}$ is the inverse of $P_{0 \rightarrow \tau}$. Here and in the sequel $\exp , \nabla, R$ always have to be understood as with respect to $g_{0}$.

Denote by $X(\tau)$ a vector field along $c_{Y_{\theta}}(\tau)$, then

$$
\begin{aligned}
\left.\frac{d}{d \tau}\right|_{\tau=0} P_{-\left(Y_{\theta}+\tau\left(Y_{2}-Y_{1}\right)\right)} X(\tau)= & \lim _{\tau \rightarrow 0} \frac{1}{\tau}\left[P_{-\left(Y_{\theta}+\tau\left(Y_{2}-Y_{1}\right)\right)} X(\tau)-P_{-Y_{\theta}} X(0)\right] \\
= & \lim _{\tau \rightarrow 0} \frac{1}{\tau}\left[\left(P_{-\left(Y_{\theta}+\tau\left(Y_{2}-Y_{1}\right)\right)} P_{0 \rightarrow \tau}-P_{-Y_{\theta}}\right) X(0)\right] \\
& +\lim _{\tau \rightarrow 0} \frac{1}{\tau}\left[P_{-\left(Y_{\theta}+\tau\left(Y_{2}-Y_{1}\right)\right)} P_{0 \rightarrow \tau}\left(P_{\tau \rightarrow 0} X(\tau)-X(0)\right)\right] \\
= & \lim _{\tau \rightarrow 0} \frac{1}{\tau}\left[\left(P_{-\left(Y_{\theta}+\tau\left(Y_{2}-Y_{1}\right)\right)} P_{0 \rightarrow \tau}-P_{-Y_{\theta}}\right) X(0)\right] \\
& +\left.P_{-Y_{\theta}} \nabla_{c_{Y_{\theta}}^{\prime}} X\right|_{\tau=0} .
\end{aligned}
$$

For $X(\tau)=\operatorname{tr}_{g_{0}} \nabla^{g_{t}} d\left(\exp \left(Y_{\theta}+\tau\left(Y_{2}-Y_{1}\right)\right)\right),(7.60)$ becomes

$$
P_{-Y_{\theta}} \nabla_{c_{Y_{\theta}}^{\prime}} \nabla^{g_{t}} d\left(\exp Y_{\theta}\right) \text {. }
$$

$c_{Y_{\theta}}(\tau)=\exp Y_{\theta}+\tau\left(Y_{2}-Y_{1}\right)$ is the image of the straight line $\tau \rightarrow Y_{\theta}+\tau\left(Y_{2}-Y_{1}\right) \subset$ $T_{f_{t_{0}}(x)} M$ under the exponential map w.r.t. $g_{0}$. Hence the tangent vector at $\tau=0$ is

$$
d\left(\exp Y_{\theta}\right)\left(\left.\frac{d}{d \tau}\right|_{\tau=0}\left(Y_{\theta}+\tau\left(Y_{2}-Y_{1}\right)\right)\right)=d\left(\exp Y_{\theta}\right)\left(Y_{2}-Y_{1}\right) .
$$

But the latter is $J(1)$, where $J(s)$ is the Jacobi field along $s \rightarrow \exp s Y_{\theta}$ with $J(0)=0, J^{\prime}(0)=Y_{2}-Y_{1}$.

Quite parallel to (7.13), we calculate

$$
\begin{aligned}
& \nabla_{c_{Y_{\theta}}^{\prime}} t_{g_{0}} \nabla^{g_{t}} d\left(\exp Y_{\theta}\right) \\
= & \operatorname{rr}_{g_{0}} \nabla_{c_{Y_{\theta}}^{\prime}} \nabla^{g_{t}} d\left(\exp Y_{\theta}\right) \\
= & g_{0}^{i j} \nabla_{c_{Y_{\theta}}^{\prime}}\left(\nabla^{g_{t}}\right){ }_{j} d\left(\exp Y_{\theta}\right)\left(e_{i}\right) \\
= & g_{0}^{i j} \nabla_{c_{Y_{\theta}}^{\prime}} \nabla_{j} d\left(\exp Y_{\theta}\right)\left(e_{i}\right)+g_{0}^{i j} \nabla_{c_{Y_{\theta}}^{\prime}}\left(\nabla^{g_{t}}-\nabla^{g_{0}}\right)_{j} d\left(\exp Y_{\theta}\right)\left(e_{i}\right) \\
= & g_{0}^{i j} \nabla_{j} \nabla_{{c_{Y_{\theta}}}^{\prime}} d\left(\exp Y_{\theta}\right)\left(e_{i}\right)+g_{0}^{i j} R^{g_{0}}\left(c_{Y_{\theta}}^{\prime}, d\left(\exp Y_{\theta}\right)\left(e_{j}\right)\right) d\left(\exp Y_{\theta}\right)\left(e_{i}\right) \\
& +g_{0}^{i j} \nabla_{{C_{Y_{\theta}}}^{\prime}}\left(\nabla^{g_{t}}-\nabla^{g_{0}}\right)_{j} d\left(\exp Y_{\theta}\right)\left(e_{i}\right) \\
= & g_{0}^{i j} \nabla_{j} \nabla_{i} c_{Y_{\theta}}^{\prime}+g_{0}^{i j} R^{g_{0}}\left(c_{Y_{\theta}}^{\prime}, d\left(\exp Y_{\theta}\right)\left(e_{j}\right)\right) d\left(\exp Y_{\theta}\right)\left(e_{i}\right) \\
& +g_{0}^{i j} \nabla_{c_{Y_{\theta}}^{\prime}}\left(\nabla^{g_{t}}-\nabla^{g_{0}}\right)_{j} d\left(\exp Y_{\theta}\right)\left(e_{i}\right),
\end{aligned}
$$

where again we used $\nabla_{s} \frac{\partial}{\partial t} c(s, t)=\nabla_{t} \frac{\partial}{\partial s} c(s, t)$.

Hence,

$$
\begin{aligned}
& F_{X}\left(t_{0}, 0\right)\left(Y_{2}-Y_{1}\right)-P_{-Y_{\theta}} \nabla_{c_{Y_{\theta}}^{\prime}} \operatorname{tr}_{g_{0}} \nabla^{g_{t}}\left(\operatorname{dexp} Y_{\theta}\right) \\
& =-\Delta_{f_{t_{0}}}\left(Y_{2}-Y_{1}\right)+t r_{g_{0}} R^{g_{t_{0}}}\left(Y_{2}-Y_{1}, d f_{t_{0}}\right) d f_{t_{0}}-P_{-Y_{\theta}} \nabla_{c_{Y_{\theta}}^{\prime}} t_{g_{0}} \nabla^{g_{t}} d\left(\exp Y_{\theta}\right) \\
& =-\Delta_{f_{t_{0}}}\left(Y_{2}-Y_{1}\right)+P_{-Y_{\theta}} \Delta_{\exp Y_{\theta}} c_{Y_{\theta}}^{\prime}+t r_{g_{0}} R^{g_{t_{0}}}\left(Y_{2}-Y_{1}, d f_{t_{0}}\right) d f_{t_{0}} \\
& -P_{-Y_{\theta}} t_{g_{0}} R^{g_{0}}\left(c_{Y_{\theta}}^{\prime}, d\left(\exp Y_{\theta}\right)\right) d\left(\exp Y_{\theta}\right)-P_{-Y_{\theta}} g_{0}^{i j} \nabla_{{c_{Y}^{\prime}}_{Y_{\theta}}}\left(\nabla^{g_{t}}-\nabla^{g_{0}}\right)_{j} d\left(\exp Y_{\theta}\right)\left(e_{i}\right) .
\end{aligned}
$$


Note that

$$
\begin{aligned}
-\Delta_{f_{t_{0}}}\left(Y_{2}-Y_{1}\right)+P_{-Y_{\theta}} \Delta_{\exp Y_{\theta}} c_{Y_{\theta}}^{\prime} & =\int_{0}^{1} \frac{d}{d s}\left[P_{-s Y_{\theta}} \Delta_{\exp s Y_{\theta}} d\left(\exp s Y_{\theta}\right)\left(Y_{2}-Y_{1}\right)\right] d s \\
& =\int_{0}^{1} P_{-s Y_{\theta}} \nabla_{c^{\prime}}\left(\Delta_{\exp s Y_{\theta}} d\left(\exp s Y_{\theta}\right)\left(Y_{2}-Y_{1}\right)\right) d s,
\end{aligned}
$$

where $c(s)=\exp s Y_{\theta}, c^{\prime}=P_{s Y_{\theta}} Y_{\theta}$, and $\nabla_{c^{\prime}} \equiv \nabla_{s} \equiv \nabla_{s}^{g_{0}}$.

We would like to estimate as in (7.13)-(7.33), but unfortunately we have here

$$
P_{-s Y_{\theta}} \nabla_{c^{\prime}}\left(\Delta_{\exp s Y_{\theta}} d\left(\exp s Y_{\theta}\right)\left(Y_{2}-Y_{1}\right)\right)
$$

rather than

$$
P_{-s Y_{\theta}} \nabla_{c^{\prime}}\left(\Delta_{\exp s Y_{\theta}} P_{s Y_{\theta}}\left(Y_{2}-Y_{1}\right)\right) .
$$

For this reason, we write

$$
\begin{gathered}
P_{-s Y_{\theta}} \nabla_{c^{\prime}}\left(\Delta_{\exp s Y_{\theta}} d\left(\exp s Y_{\theta}\right)\left(Y_{2}-Y_{1}\right)\right) \\
=P_{-s Y_{\theta}} \nabla_{c^{\prime}}\left(\Delta_{\exp s Y_{\theta}} P_{s Y_{\theta}}\left(Y_{2}-Y_{1}\right)\right) \\
+P_{-s Y_{\theta}} \nabla_{c^{\prime}}\left(\Delta_{\exp s Y_{\theta}}\left[d\left(\exp s Y_{\theta}\right)\left(Y_{2}-Y_{1}\right)-P_{s Y_{\theta}}\left(Y_{2}-Y_{1}\right)\right] .\right.
\end{gathered}
$$

If we replace $Y$ by $Y_{2}-Y_{1}$ in (7.13)-(7.33), then we get

$$
\left|P_{-Y_{\theta}} \Delta_{\exp Y_{\theta}} P_{Y_{\theta}}\left(Y_{2}-Y_{1}\right)-\Delta_{f_{t_{0}}}\left(Y_{2}-Y_{1}\right)\right|_{r-2} \leq p_{r-1}\left(\left|Y_{2}-Y_{1}\right|_{r}\right)\left|Y_{2}-Y_{1}\right|_{r}
$$

We write

$$
\left[d\left(\exp s Y_{\theta}\right)\right] s\left(Y_{2}-Y_{1}\right)-P_{s Y_{\theta}}\left(Y_{2}-Y_{1}\right):=J(s)-P_{s}\left(Y_{2}-Y_{1}\right)
$$

and have to estimate

$$
P_{-Y_{\theta}} \nabla_{c^{\prime}} \Delta_{\exp Y_{\theta}}\left(J(1)-P_{1}\left(Y_{2}-Y_{1}\right)\right) .
$$

The zero-th step is to estimate $J(1)-P_{1}\left(Y_{2}-Y_{1}\right)$. Consider for this $c(s, \tau)=\exp s\left(Y_{\theta}+\right.$ $\left.\tau\left(Y_{2}-Y_{1}\right)\right), c^{\prime}(s)=\left.\frac{\partial}{\partial s} c(s, \tau)\right|_{\tau=0}=P_{s Y_{\theta}} Y_{\theta},{ }^{\prime} \equiv \nabla_{s} \equiv \nabla_{s}^{g_{0}}$ and the equation

$$
\left(J(s)-P_{s} J^{\prime}(0)-s J^{\prime}(s)\right)^{\prime}=s R\left(J, c^{\prime}\right) c^{\prime},
$$

which implies for the pointwise norms

$$
\begin{aligned}
&\left|J(s)-P_{s} J^{\prime}(0)-s J^{\prime}(s)\right|^{\prime} \leq\left|\left(J(s)-P_{s} J^{\prime}(0)-s J^{\prime}(s)\right)^{\prime}\right| \leq s\left|R\left(J, c^{\prime}\right) c^{\prime}\right|, \\
&\left|J(1)-P_{1} J^{\prime}(0)-J^{\prime}(1)\right|-\left|J^{\prime}(0)\right| \leq \int_{0}^{1} s|R||J|\left|c^{\prime}\right|^{2} \\
& \leq \frac{\sqrt{2}}{2} \max |J(s)|\left|Y_{\theta}\right|^{2} \\
& \leq \max |J(s)|\left(\left|Y_{1}\right|+\left|Y_{2}-Y_{1}\right|\right)^{2} \\
&= \mid \frac{\sinh \left|Y_{\theta}\right|}{\left|Y_{\theta}\right|} P_{1}\left(Y_{2}-Y_{1}\right)^{(n)} \\
&+P_{1}\left(Y_{2}-Y_{1}\right)^{(t)} \mid\left(\left|Y_{1}\right|+\left|Y_{2}-Y_{1}\right|\right)^{2} \\
& \leq 3\left(\left|Y_{1}\right|+\left|Y_{2}-Y_{1}\right|\right)^{2}\left|Y_{2}-Y_{1}\right| .
\end{aligned}
$$


We obtain from

$$
J(s)=\frac{\sinh \left(s\left|Y_{\theta}\right|\right)}{\left|Y_{\theta}\right|} P_{s}\left(Y_{2}-Y_{1}\right)^{(n)}+s P_{s}\left(Y_{2}-Y_{1}\right)^{(t)}
$$

that

$$
J^{\prime}(s)=\cosh \left(s\left|Y_{\theta}\right|\right) P_{s}\left(Y_{2}-Y_{1}\right)^{(n)}+P_{s}\left(Y_{2}-Y_{1}\right)^{(t)},
$$

and $J^{\prime}(0)=Y_{2}-Y_{1}$ as it should be. (7.68)-(7.70) yield

$$
\begin{aligned}
\left|J(1)-P_{1} J^{\prime}(0)\right| \leq & \left|J^{\prime}(0)\right|+\left|J^{\prime}(1)\right|+\int_{0}^{1} s|R||J|\left|c^{\prime}\right|^{2} d s \\
\leq & \left|Y_{2}-Y_{1}\right|+|\cosh | Y_{\theta} P_{1}\left(Y_{2}-Y_{1}\right)^{(n)}+P_{1}\left(Y_{2}-Y_{1}\right)^{(t)} \mid \\
& +3\left(\left|Y_{1}\right|+\left|Y_{2}-Y_{1}\right|\right)^{2}\left|Y_{2}-Y_{1}\right| \\
\leq & \left.C\left(1+\left|Y_{1}\right|+\left|Y_{2}-Y_{1}\right|\right)^{2}\left|Y_{2}-Y_{1}\right|\right) .
\end{aligned}
$$

We have to calculate the derivative $\nabla_{c^{\prime}} \Delta\left(J(1)-P_{1}\left(Y_{2}-Y_{1}\right)\right)$ and start with the equation ( with $^{\prime}=\nabla_{s}=\nabla_{c^{\prime}}$ )

$$
\begin{aligned}
& \left(\nabla_{Z} J(s)-\nabla_{Z} P_{s} J^{\prime}(0)-s \nabla_{Z} J^{\prime}(s)\right)^{\prime} \\
= & \nabla_{s} \nabla_{Z} J-\nabla_{s} \nabla_{Z} P_{s} J^{\prime}(0)-\nabla_{Z} \nabla_{s} J-s \nabla_{s} \nabla_{Z} \nabla_{s} J \\
= & \nabla_{s} \nabla_{Z} J-\nabla_{Z} \nabla_{s} J-\nabla_{s} \nabla_{Z} P_{s} J^{\prime}(0)-s \nabla_{s} \nabla_{Z} \nabla_{s} J \\
= & R\left(c^{\prime}, Z\right) J-\nabla_{Z} \nabla_{s} P_{s} J^{\prime}(0)-R\left(c^{\prime}, Z\right) P_{s} J^{\prime}(0)-s \nabla_{s} \nabla_{s}\left(\nabla_{Z} J\right)-s \nabla_{s} R\left(Z, c^{\prime}\right) J \\
= & R\left(c^{\prime}, Z\right) J-R\left(c^{\prime}, Z\right) P_{s} J^{\prime}(0)-s \nabla_{s} \nabla_{s}\left(\nabla_{Z} J\right)-s \nabla_{s} R\left(Z, c^{\prime}\right) J \\
= & R\left(c^{\prime}, J\right)\left(J-P_{s} J^{\prime}(0)\right)+s \mathscr{R}(J, J)-s R\left(\nabla_{Z} J, c^{\prime}\right) c^{\prime}-\nabla_{s} R\left(Z, c^{\prime}\right) J
\end{aligned}
$$

where we used (cf. [10], [12])

$$
\begin{aligned}
& \nabla_{s} \nabla_{s}\left(\nabla_{Z} J\right)+R\left(\nabla_{Z} J, c^{\prime}\right) c^{\prime}=-\mathscr{R}(Z, J), \\
\mathscr{R}(X, Y) \equiv & \left(\nabla_{s} R\right)\left(X, c^{\prime}\right) Y+\left(\nabla_{Z} R\right)\left(Y, c^{\prime}\right) c^{\prime}+2 R\left(X, c^{\prime}\right) \nabla_{s} Y \\
& +2 R\left(Y, c^{\prime}\right) \nabla_{s} X
\end{aligned}
$$

and assume $|Z|=1,\left|\nabla_{c^{\prime}} Z\right|$ bounded. Hence we obtain

$$
\begin{aligned}
\left|\nabla_{Z} J-\nabla_{Z} P_{s} J^{\prime}(0)-s \nabla_{Z} J^{\prime}\right|^{\prime} \leq\left|\left(\nabla_{Z} J-\nabla_{Z} P_{s} J^{\prime}(0)-s \nabla_{Z} J^{\prime}\right)^{\prime}\right| \\
=\left|R\left(c^{\prime}, J\right)\left(J-P_{s} J^{\prime}(0)\right)+s \mathscr{R}(J, J)-s R\left(\nabla_{Z} J, c^{\prime}\right) c^{\prime}-\nabla_{s} R\left(Z, c^{\prime}\right) J\right|, \\
\left|\nabla_{Z}\left(J(1)-P_{1} J^{\prime}(0)\right)\right| \equiv \\
\leq\left|\nabla_{Z}\left[\left(d\left(\exp Y_{\theta}\right)\right)\left(Y_{2}-Y_{1}\right)-P_{Y_{\theta}}\left(Y_{2}-Y_{1}\right)\right]\right| \\
\leq\left|\nabla_{Z} J^{\prime}(1)\right|+\left|\nabla_{Z} J^{\prime}(0)\right|+\int_{0}^{1} \mid R\left(c^{\prime}, J\right)\left(J-P_{s} J^{\prime}(0)\right) \\
+s \mathscr{R}(J, J)-s R\left(\nabla_{Z} J, c^{\prime}\right) c^{\prime}-\nabla_{s} R\left(Z, c^{\prime}\right) J \mid d s .
\end{aligned}
$$

We estimate the single terms of (7.75).

$$
\nabla_{Z} J^{\prime}(0)=\nabla_{Z}\left(Y_{2}-Y_{1}\right)
$$




$$
\begin{aligned}
\nabla_{Z} J^{\prime}(1)= & \nabla_{Z}\left[\cosh \left(\left|Y_{\theta}\right|\right) P_{1}\left(Y_{2}-Y_{1}\right)^{(n)}+P_{1}\left(Y_{2}-Y_{1}\right)^{(t)}\right] \\
= & \sinh \left(\left|Y_{\theta}\right|\right) \nabla_{Z}\left|Y_{\theta}\right| P_{1}\left(Y_{2}-Y_{1}\right)^{(n)}+\cosh \left(\left|Y_{\theta}\right|\right)\left(\nabla_{Z} P_{1}\right)\left(Y_{2}-Y_{1}\right)^{(n)} \\
& +\cosh \left(\left|Y_{\theta}\right|\right) P_{1} \nabla_{Z}\left(Y_{2}-Y_{1}\right)^{(n)}+\left(\nabla_{Z} P_{1}\right)\left(Y_{2}-Y_{1}\right)^{(t)} \\
& +P_{1} \nabla_{Z}\left(Y_{2}-Y_{1}\right)^{(t)} .
\end{aligned}
$$

Hence we obtain for $Z$ a unit vector

$$
\left|\nabla_{Z} J^{\prime}(0)\right| \leq\left|\nabla\left(Y_{2}-Y_{1}\right)\right|
$$

and

$$
\begin{aligned}
\left|\nabla_{Z} J^{\prime}(1)\right| \leq & C_{0}\left(\left|\nabla Y_{1}\right|+\left|\nabla\left(Y_{2}-Y_{1}\right)\right|\right)\left|Y_{2}-Y_{1}\right|+C_{1}\left|Y_{2}-Y_{1}\right|+C_{2}\left|\nabla\left(Y_{2}-Y_{1}\right)\right| \\
& +\left|\left(1-\cosh \left|Y_{\theta}\right|\right) \nabla_{Z}\left(Y_{2}-Y_{1}\right)^{(t)}\right| .
\end{aligned}
$$

But

$$
\begin{aligned}
\nabla_{Z}\left(Y_{2}-Y_{1}\right)^{(t)}= & \nabla_{Z}\left[\left(Y_{2}-Y_{1}, \frac{c^{\prime}}{\left|c^{\prime}\right|}\right) \frac{c^{\prime}}{\left|c^{\prime}\right|}\right] \\
= & \left(\nabla_{Z}\left(Y_{2}-Y_{1}\right), \frac{c^{\prime}}{\left|c^{\prime}\right|}\right) \frac{c^{\prime}}{\left|c^{\prime}\right|}+\left(Y_{2}-Y_{1},-\frac{1}{\left|c^{\prime}\right|^{3}}\left(\nabla c^{\prime}, c^{\prime}\right) c^{\prime}+\frac{\nabla c^{\prime}}{\left|c^{\prime}\right|}\right) \frac{c^{\prime}}{\left|c^{\prime}\right|} \\
& +\left(Y_{2}-Y_{1}, \frac{c^{\prime}}{\left|c^{\prime}\right|}\right)\left[-\frac{1}{\left|c^{\prime}\right|^{3}}\left(\nabla c^{\prime}, c^{\prime}\right) c^{\prime}+\frac{\nabla c^{\prime}}{\left|c^{\prime}\right|}\right]
\end{aligned}
$$

which immediately implies

$$
\begin{aligned}
& \left|\left(1-\cosh \left|Y_{\theta}\right|\right) \nabla_{Z}\left(Y_{2}-Y_{1}\right)^{(t)}\right| \leq C\left[\left|\nabla\left(Y_{2}-Y_{1}\right)\right|\left(\left|Y_{1}+\right| Y_{2}-Y_{1} \mid\right)^{2}\right. \\
& \left.+\left|Y_{2}-Y_{1}\right|\left(\left|\nabla Y_{1}\right|+\left|\nabla\left(Y_{2}-Y_{1}\right)\right|\right)\left(\left|Y_{1}+\right| Y_{2}-Y_{1} \mid\right)\right]
\end{aligned}
$$

and

$$
\begin{aligned}
\left|\nabla_{Z} J^{\prime}(1)\right| \leq & C\left[\left(\left|\nabla Y_{1}\right|+\left|\nabla\left(Y_{2}-Y_{1}\right)\right|\right)\left|Y_{2}-Y_{1}\right|+\left|Y_{2}-Y_{1}\right|+\left|\nabla\left(Y_{2}-Y_{1}\right)\right|\right. \\
& +\left|\nabla\left(Y_{2}-Y_{1}\right)\right|\left(\left|Y_{1}\right|+\left|Y_{2}-Y_{1}\right|\right)^{2} \\
& \left.+\left(\left|\nabla Y_{1}\right|+\left|\nabla\left(Y_{2}-Y_{1}\right)\right|\right)\left(\left|Y_{1}\right|+\left|Y_{2}-Y_{1}\right|\right)\left|Y_{2}-Y_{1}\right|\right] .
\end{aligned}
$$

We estimate the terms in (7.71).

$$
\begin{gathered}
\left|R\left(c^{\prime}, J\right)\left(J-P_{s} J^{\prime}(0)\right)\right|=2\left(\left|Y_{1}\right|+\left|Y_{2}-Y_{1}\right|\right)|J|\left|J-P_{s} J^{\prime}(0)\right| \\
\leq 12\left(\left|Y_{1}\right|+\left|Y_{2}-Y_{1}\right|\right)^{3}\left|Y_{2}-Y_{1}\right|^{2} \\
\left|R\left(\nabla_{Z} J, c^{\prime}\right) c^{\prime}\right| \leq 2 C\left(\left|Y_{1}\right|+\left|Y_{2}-Y_{1}\right|\right)^{2}\left[\left(\left|\nabla Y_{1}\right|+\left|\nabla\left(Y_{2}-Y_{1}\right)\right|\right)\left|Y_{2}-Y_{1}\right|\right. \\
\left.+\left|\nabla\left(Y_{2}-Y_{1}\right)\right|+\left(\left|\nabla Y_{1}\right|+\left|\nabla\left(Y_{2}-Y_{1}\right)\right|\right)\left(\left|Y_{1}\right|+\left|Y_{2}-Y_{1}\right|\right)\left|Y_{2}-Y_{1}\right|\right] \\
\left|\nabla_{s} R\left(Z, c^{\prime}\right) J\right| \leq\left|c^{\prime}\right|\left|\nabla R\left(Z, c^{\prime}\right) J\right| \\
\leq \sqrt{2}\left(\left|Y_{1}\right|+\left|Y_{2}-Y_{1}\right|\right) \cdot C \cdot\left[\left(\left|Y_{1}\right|+\left|Y_{2}-Y_{1}\right|\right)\left|Y_{2}-Y_{1}\right|\right. \\
\left.\quad+\left(\left|\nabla Y_{1}\right|+\left|\nabla\left(Y_{2}-Y_{1}\right)\right|\right)\left|Y_{2}-Y_{1}\right|+\left(\left|Y_{1}\right|+\left|Y_{2}-Y_{1}\right|\right)\right]
\end{gathered}
$$


We see from (7.75)-(7.85) that

$$
\begin{aligned}
\left|\nabla\left(J(1)-P_{1} J^{\prime}(0)\right)\right| \leq & P_{1}\left(\left(\left|Y_{1}\right|+\left|Y_{2}-Y_{1}\right|\right),\left(\left|\nabla Y_{1}\right|+\left|\nabla\left(Y_{2}-Y_{1}\right)\right|\right),\right. \\
& \left.\left|Y_{2}-Y_{1}\right|,\left|\nabla\left(Y_{2}-Y_{1}\right)\right|\right),
\end{aligned}
$$

where $P_{1}$ is a polynomial in the indicated variables with positive coefficients independent of $t_{0}$ and such that each monomial contains a factor $\left|Y_{2}-Y_{1}\right|$ or $\left|\nabla\left(Y_{2}-Y_{1}\right)\right|$.

Next we must estimate

$$
\begin{gathered}
\left|g_{0}^{i j} \nabla_{j} \nabla_{i}\left(J(1)-P_{1} J^{\prime}(0)\right)\right| \\
\left|\nabla_{c^{\prime}} g_{0}^{i j} \nabla_{j} \nabla_{i}\left(J(1)-P_{1} J^{\prime}(0)\right)\right|
\end{gathered}
$$

and

$$
\left|\nabla^{u} \nabla_{c^{\prime}} g_{0}^{i j} \nabla_{j} \nabla_{i}\left(J(1)-P_{1} J^{\prime}(0)\right)\right|, \quad 0 \leq u \leq r-2 .
$$

We again apply our standard formula

$$
\nabla_{Z}^{u} \nabla_{c^{\prime}} V=\nabla_{c^{\prime}} \nabla_{Z}^{u}+\sum_{i=1}^{u} \nabla_{Z}^{u-i} R\left(V, c^{\prime}\right) \nabla_{Z}^{i-1} V
$$

and estimate

$$
\nabla_{c^{\prime}} \nabla_{Z}^{u}\left(J(1)-P_{1} J^{\prime}(0)\right)+\sum_{i=1}^{u} \nabla_{Z}^{u-i} R\left(\left(J(1)-P_{1} J^{\prime}(0)\right), c^{\prime}\right) \nabla_{Z}^{i-1}\left(J(1)-P_{1} J^{\prime}(0)\right) .
$$

Suppose $\nabla_{Z}^{u-1}\left(J(1)-P_{1} J^{\prime}(0)\right)$ can be estimated by a polynomial $Q_{u-1}\left(\left(\left|Y_{1}\right|+\mid Y_{2}-\right.\right.$ $\left.\left.Y_{1} \mid\right), \cdots,\left(\left|\nabla^{u-1} Y_{1}\right|+\left|\nabla^{u-1}\left(Y_{2}-Y_{1}\right)\right|\right),\left|Y_{2}-Y_{1}\right|, \cdots,\left|\nabla^{u-1}\left(Y_{2}-Y_{1}\right)\right|\right)$ in the indicated variables with positive coefficients independent of $t_{0}$ and such that each monomial contains a factor $\left|\nabla^{j}\left(Y_{2}-Y_{1}\right)\right|$. Then, as immediately follows from the chain and Leibniz rule

$$
\sum_{i=1}^{u} \nabla_{Z}^{u-i} R\left(\left(J(1)-P_{1} J^{\prime}(0)\right), c^{\prime}\right) \nabla_{Z}^{i-1}\left(J(1)-P_{1} J^{\prime}(0)\right) \leq Q_{u-1}(\cdots) .
$$

There remains to investigate $\nabla_{Z}^{u}\left(J(1)-P_{1} J^{\prime}(0)\right)$. For this, we consider the expression

$$
\begin{aligned}
& {\left[\nabla_{Z}^{u}\left(J(s)-\nabla_{Z}^{u} P_{s} J^{\prime}(0)-s \nabla_{Z}^{u} J^{\prime}(s)\right]^{\prime}\right.} \\
& =-\sum_{i=1}^{u} \nabla_{Z}^{u-i} R\left(\left(J(s), c^{\prime}\right) \nabla_{Z}^{i-1} J(s)-\nabla_{Z}^{u} \nabla_{s} P_{s} J^{\prime}(0)\right. \\
& +\sum_{i=1}^{u} \nabla_{Z}^{u-i} R\left(P_{s} J^{\prime}(0), c^{\prime}\right) \nabla_{Z}^{i-1} P_{s} J^{\prime}(0) \\
& -\nabla_{Z}^{u} \nabla_{s} J(s)-s\left[\nabla_{s} \nabla_{s} \nabla_{Z}^{u} J(s)+\sum_{i=1}^{u} \nabla_{Z}^{u-i} R\left(J(s), c^{\prime}\right) \nabla_{Z}^{i-1} P_{s} J(s)\right] \\
& =-(1+s) \sum_{i=1}^{u} \nabla_{Z}^{u-i} R\left(\left(J(s), c^{\prime}\right) \nabla_{Z}^{i-1} J(s)\right. \\
& +\sum_{i=1}^{u} \nabla_{Z}^{u-i} R\left(P_{s} J^{\prime}(0), c^{\prime}\right) \nabla_{Z}^{i-1} P_{s} J^{\prime}(0)-\nabla_{Z}^{u} \nabla_{s} P_{s} J^{\prime}(0) \\
& +s\left[R\left(\nabla_{Z}^{u} J(s), c^{\prime}\right) c^{\prime}+\mathscr{R}\left(Z, \nabla_{Z}^{u-2} J\right)+\nabla_{Z} \mathscr{R}\left(Z, \nabla_{Z}^{u-3} J\right)+\cdots+\nabla_{Z}^{u-2} \mathscr{R}(Z, J)\right],
\end{aligned}
$$


since

$$
\nabla_{s} \nabla_{Z}^{u} J=\nabla_{Z}^{u} \nabla_{s} J-\sum_{i=1}^{u} \nabla_{Z}^{u-i} R\left(J, c^{\prime}\right) \nabla_{Z}^{i-1} J
$$

and (cf. [10])

$$
\begin{aligned}
0= & \nabla_{s} \nabla_{s} \nabla_{Z}^{u} J+R\left(\nabla_{Z}^{u} J(s), c^{\prime}\right) c^{\prime}+\mathscr{R}\left(Z, \nabla_{Z}^{u-2} J\right) \\
& +\nabla_{Z} \mathscr{R}\left(Z, \nabla_{Z}^{u-3} J\right)+\cdots+\nabla_{Z}^{u-2} \mathscr{R}(Z, J) .
\end{aligned}
$$

Clearly, $\nabla_{Z}^{u} \nabla_{s} P_{s} J^{\prime}(0)=0$. We start with the estimate of the single terms in (7.92).

$$
\begin{gathered}
-\sum_{i=1}^{u} \nabla_{Z}^{u-i} R\left(\left(J(s), c^{\prime}\right) \nabla_{Z}^{i-1} J(s)+\sum_{i=1}^{u} \nabla_{Z}^{u-i} R\left(P_{s} J^{\prime}(0), c^{\prime}\right) \nabla_{Z}^{i-1} P_{s} J^{\prime}(0)\right. \\
=\sum_{i=1}^{u} \nabla_{Z}^{u-i} R\left(P_{s} J^{\prime}(0)-J(s), c^{\prime}\right) \nabla_{Z}^{i-1} P_{s} J^{\prime}(0) \\
+\sum_{i=1}^{u} \nabla_{Z}^{u-i} R\left(J(s), c^{\prime}\right) \nabla_{Z}^{i-1}\left(P_{s} J^{\prime}(0)-J(s)\right)
\end{gathered}
$$

(7.93) or (7.94) is a sum of terms of the kind

$$
R\left(\nabla_{Z}^{i_{1}}\left(P_{s} J^{\prime}(0)-J(s)\right), \nabla_{Z}^{i_{2}} c^{\prime}\right) \nabla_{Z}^{i_{3}} P_{s} J^{\prime}(0)
$$

or

$$
R\left(\nabla_{Z}^{i_{1}}\left(J(s), \nabla_{Z}^{i_{2}} c^{\prime}\right) \nabla_{Z}^{i_{3}}\left(P_{s} J^{\prime}(0)-J(s)\right),\right.
$$

$i_{1}+i_{2}+i_{3}=u-1$ respectively.

(7.95) can be estimated as follows.

$$
\begin{gathered}
\left|R\left(\nabla_{Z}^{i_{1}}\left(P_{s} J^{\prime}(0)-J(s)\right), \nabla_{Z}^{i_{2}} c^{\prime}\right) \nabla_{Z}^{i_{3}} P_{s} J^{\prime}(0)\right| \\
\leq \sqrt{2}\left|\nabla_{Z}^{i_{1}}\left(P_{s} J^{\prime}(0)-J(s)\right)\right|\left|\nabla_{Z}^{i_{2}} c^{\prime}\right|\left|\nabla_{Z}^{i_{3}} P_{s} J^{\prime}(0)\right| \\
\left|\nabla_{Z}^{i_{1}}\left(P_{s} J^{\prime}(0)-J(s)\right)\right| \leq Q_{i_{1}}^{(1)}\left(\left(\left|Y_{1}\right|+\left|Y_{2}-Y_{1}\right|\right), \cdots,\left|\nabla^{i_{1}}\left(Y_{2}-Y_{1}\right)\right|\right), \\
\left|\nabla_{Z}^{i_{2}} c^{\prime}\right| \leq Q_{i_{2}}^{(2)}\left(\left(\left|Y_{1}\right|+\left|Y_{2}-Y_{1}\right|\right), \cdots,\left(\left|\nabla^{i_{2}} Y_{1}\right|+\left|\nabla^{i_{2}}\left(Y_{2}-Y_{1}\right)\right|\right)\right), \\
\left|\nabla_{Z}^{i_{3}} P_{s} J^{\prime}(0)\right| \leq Q_{i_{3}}^{(3)}\left(\left|Y_{2}-Y_{1}\right|, \cdots,\left|\nabla^{i_{3}}\left(Y_{2}-Y_{1}\right)\right|\right)
\end{gathered}
$$

and (7.96) as

$$
\begin{gathered}
\mid R\left(\nabla_{Z}^{i_{1}}\left(J(s), \nabla_{Z}^{i_{2}} c^{\prime}\right) \nabla_{Z}^{i_{3}}\left(P_{s} J^{\prime}(0)-J(s)\right) \mid\right. \\
\leq\left|\sqrt{2} \nabla_{Z}^{i_{1}} J(s)\right|\left|\nabla_{Z}^{i_{2}} c^{\prime}\right|\left|\nabla_{Z}^{i_{3}} P_{s} J^{\prime}(0)\right|, \\
\left|\nabla_{Z}^{i_{2}} c^{\prime}\right| \leq Q_{i_{2}}^{(2)}\left(\left(\left|Y_{1}\right|+\left|Y_{2}-Y_{1}\right|\right), \cdots,\left(\left|\nabla^{i_{2}} Y_{1}\right|+\left|\nabla^{i_{2}}\left(Y_{2}-Y_{1}\right)\right|\right)\right),
\end{gathered}
$$




$$
\left|\nabla_{Z}^{i_{3}} P_{s} J^{\prime}(0)-J(s)\right| \leq Q_{i_{3}}^{(1)}\left(\left(\left|Y_{1}\right|+\left|Y_{2}-Y_{1}\right|\right), \cdots,\left|\nabla^{i_{3}}\left(Y_{2}-Y_{1}\right)\right|\right) .
$$

We need to estimate $\nabla_{Z}^{i_{1}} J(s)$.

$$
\begin{aligned}
& \nabla_{Z} J(s) \\
= & \left.\nabla_{Z}\left[\frac{\sinh \left(s\left|Y_{\theta}\right|\right)}{\left|Y_{\theta}\right|} P_{s}\left(Y_{2}-Y_{1}\right)^{(n)}+s P_{s}\left(Y_{2}-Y_{1}\right)^{(t)}\right)\right] \\
= & \frac{\cosh \left(s\left|Y_{\theta}\right|\right)}{\left|Y_{\theta}\right|} s \nabla_{Z}\left|Y_{\theta}\right| P_{s}\left(Y_{2}-Y_{1}\right)^{(n)}+\frac{\sinh \left(s\left|Y_{\theta}\right|\right)}{\left|Y_{\theta}\right|}\left(\nabla_{Z} P_{s}\right)\left(Y_{2}-Y_{1}\right)^{(n)} \\
& +\frac{\sinh \left(s\left|Y_{\theta}\right|\right)}{\left|Y_{\theta}\right|} P_{s} \nabla_{Z}\left(Y_{2}-Y_{1}\right)^{(n)}+s\left(\nabla_{Z} P_{s}\right)\left(Y_{2}-Y_{1}\right)^{(t)}+s P_{s}\left(\nabla_{Z}\left(Y_{2}-Y_{1}\right)^{(t)}\right) \\
= & \frac{\cosh \left(s\left|Y_{\theta}\right|\right)}{\left|Y_{\theta}\right|} s \nabla_{Z}\left|Y_{\theta}\right| P_{s}\left(Y_{2}-Y_{1}\right)^{(n)}+\frac{\sinh \left(s\left|Y_{\theta}\right|\right)}{\left|Y_{\theta}\right|}\left[\left(\nabla_{Z} P_{s}\right)\left(Y_{2}-Y_{1}\right)\right. \\
& \left.+P_{s} \nabla_{Z}\left(Y_{2}-Y_{1}\right)\right]+\frac{s\left|Y_{\theta}\right|-\sinh \left(s\left|Y_{\theta}\right|\right)}{\left|Y_{\theta}\right|}\left[\left(\nabla_{Z} P_{s}\right)\left(Y_{2}-Y_{1}\right)^{(t)}\right. \\
& \left.+P_{s} \nabla_{Z}\left(Y_{2}-Y_{1}\right)^{(t)}\right] .
\end{aligned}
$$

We estimate $\left|\nabla_{Z}\left(Y_{2}-Y_{1}\right)^{(t)}\right|\left|Y_{\theta}\right|^{2}$ according to (7.80):

$\left|\nabla_{Z}\left(Y_{2}-Y_{1}\right)^{(t)}\right|\left|Y_{\theta}\right|^{2} \leq C\left|\nabla\left(Y_{2}-Y_{1}\right)\right|\left(\left|Y_{1}\right|+\left|Y_{2}-Y_{1}\right|\right)+\left|Y_{2}-Y_{1}\right|\left(\left|Y_{1}\right|+\left|Y_{2}-Y_{1}\right|\right)$,

then

$$
\begin{aligned}
\left|\nabla_{Z} J(s)\right| \leq & C \frac{\cosh \left(\left|Y_{\theta}\right|\right)}{\left|Y_{\theta}\right|}\left(\left|\nabla Y_{1}\right|+\left|\nabla\left(Y_{2}-Y_{1}\right)\right|\right)\left|Y_{2}-Y_{1}\right|+\left|Y_{2}-Y_{1}\right| \\
& +\left|\nabla\left(Y_{2}-Y_{1}\right)\right|+\left(\left|Y_{1}\right|+\left|Y_{2}-Y_{1}\right|\right)^{2}\left|Y_{2}-Y_{1}\right| \\
& +\left|\nabla\left(Y_{2}-Y_{1}\right)\right|\left(\left|Y_{1}\right|+\left|Y_{2}-Y_{1}\right|\right)+\left(\left|Y_{1}\right|+\left|Y_{2}-Y_{1}\right|\right)\left|Y_{2}-Y_{1}\right| \\
= & Q_{1}^{(4)}\left(\left(\left|Y_{1}\right|+\left|Y_{2}-Y_{1}\right|\right),\left(\left|\nabla Y_{1}\right|+\left|\nabla\left(Y_{2}-Y_{1}\right)\right|\right),\left|Y_{2}-Y_{1}\right|,\left|\nabla\left(Y_{2}-Y_{1}\right)\right|\right),
\end{aligned}
$$

where $Q_{1}^{(4)}$ is a polynomial in the indicated variables with positive coefficients independent of $t_{0}$ without constant term and such that each term contains $\left|Y_{2}-Y_{1}\right|$ or $\left|\nabla\left(Y_{2}-Y_{1}\right)\right|$ as a factor.

To estimate $\nabla_{Z}^{i} J(s)$, we have to differentiate (7.104). A simple induction and again the chain and Leibniz rule yield an estimate

$$
\left|\nabla_{Z}^{i} J(s)\right| \leq Q_{1}^{(4)}\left(\left(\left|Y_{1}\right|+\left|Y_{2}-Y_{1}\right|\right), \cdots,\left|\nabla^{i}\left(Y_{2}-Y_{1}\right)\right|\right),
$$

where $Q_{i}^{(4)}$ is a polynomial with analogous properties as $Q_{1}^{(4)}$. Here we remark that $\frac{1}{\left|c^{\prime}\right|}$ in $\frac{1}{\left|c^{\prime}\right|^{3}}\left(\nabla c^{\prime}, c^{\prime}\right) c^{\prime}$ and $\frac{\nabla c^{\prime}}{\left|c^{\prime}\right|}$ disappears since we have to multiply with $\left|c^{\prime}\right|^{2}$ (which comes from $\left.\left|\frac{s\left|Y_{\theta}\right|-\sinh \left(s\left|Y_{\theta}\right|\right)}{\left|Y_{\theta}\right|}\right|\right)$.

We remark further that $Q_{i}^{(4)}$ contains monomials of degree one, namely certain $\left|\nabla\left(Y_{2}-Y_{1}\right)\right|$.

We accomplished the pointwise estimate of the terms (7.93) and (7.94).

The last step is the estimate of (7.92) which is the sum of terms

$$
\begin{aligned}
\nabla_{Z}^{i-2} \mathscr{R}\left(Z, \nabla_{Z}^{u-i} J\right)= & \nabla_{Z}^{i-2}\left(\nabla_{s} R\right)\left(Z, c^{\prime}\right) \nabla_{Z}^{u-i} J+\nabla^{i-2}\left(\nabla_{Z} R\right)\left(\nabla_{Z}^{u-i} J, c^{\prime}\right) c^{\prime} \\
& +2 \nabla_{Z}^{i-2} R\left(Z, c^{\prime}\right) \nabla_{s} \nabla_{Z}^{u-i} J+2 \nabla_{Z}^{i-2} R\left(\nabla^{u-i} J, c^{\prime}\right) \nabla_{s} Z \\
= & 2 \nabla_{Z}^{i-2} R\left(Z, c^{\prime}\right) \nabla_{s} \nabla_{Z}^{u-i} J
\end{aligned}
$$


since $\nabla_{Z} R=0=\nabla_{s} R$ and we assume $Z$ to be parallel along $c(s)=\exp s Y_{\theta}$, moreover, $|Z|=1$. (7.106) splits into a sum of terms of the kind

$$
2 R\left(\nabla_{Z}^{i_{1}} Z, \nabla_{Z}^{i_{2}} c^{\prime}\right) \nabla^{i_{3}} \nabla_{s} \nabla_{Z}^{u-i} J
$$

$i_{1}+i_{2}+i_{3}=i-2$. We assume $\left|\nabla_{Z}^{i_{1}} Z\right| \leq C^{\prime}, i_{2} \leq i-2$.

Hence

$$
\begin{aligned}
& \left|R\left(\nabla_{Z}^{i_{1}} Z, \nabla_{Z}^{i_{2}} c^{\prime}\right) \nabla_{Z}^{i_{3}} \nabla_{s} \nabla_{Z}^{u-i} J\right| \leq C^{\prime}\left|\nabla_{Z}^{i_{2}} c^{\prime}\right|\left|\nabla_{Z}^{i_{3}} \nabla_{s} \nabla_{Z}^{u-i} J\right| \\
& \leq C Q_{i_{2}}^{(2)}\left(\left(\left|Y_{1}\right|+\left|Y_{2}-Y_{1}\right|\right), \cdots,\left(\left|\nabla^{i_{2}} Y_{1}\right|+\left|\nabla^{i_{2}}\left(Y_{2}-Y_{1}\right)\right|\right)\right)\left|\nabla_{Z}^{i_{3}} \nabla_{s} \nabla_{Z}^{u-i} J\right|,
\end{aligned}
$$

we note that

$$
\begin{aligned}
\left|\nabla_{Z}^{i_{3}} \nabla_{s} \nabla_{Z}^{u-i} J\right| & =\left|\nabla_{s} \nabla_{Z}^{i_{3}} \nabla_{Z}^{u-i} J+\sum_{j=1}^{i_{3}} \nabla_{Z}^{i_{3}-j} R\left(\nabla_{Z}^{u-i} J, c^{\prime}\right) \nabla_{Z}^{j-1} \nabla_{Z}^{u-i} J\right| \\
\leq & \left|\nabla_{s} \nabla_{Z}^{u-i+i_{3}} J\right|+\sum_{j=1}^{i_{3}} \nabla_{Z}^{i_{3}-j} R\left(\nabla_{Z}^{u-i} J, c^{\prime}\right) \nabla_{Z}^{u-i+j-1} J \mid, \\
\left|\nabla_{s} \nabla_{Z}^{u-i+i_{3}} J\right| \leq & \left|c^{\prime}\right|\left|\nabla\left(\nabla_{Z}^{u-i+i_{3}} J\right)\right| \\
\leq & \left(\left|Y_{1}\right|+\left|Y_{2}-Y_{1}\right|\right) C Q_{u-i+i_{3}+1}^{(4)}\left(\left(\left|Y_{1}\right|+\left|Y_{2}-Y_{1}\right|\right), \cdots,\right. \\
& \left.\left|\nabla^{u-i+i_{3}+1}\left(Y_{2}-Y_{1}\right)\right|\right),
\end{aligned}
$$

since $|\nabla X|=\left(\sum_{i}\left|\nabla_{e_{i}} X\right|^{2}\right)^{\frac{1}{2}}$ and in our case $Z$ runs through $\left\{e_{i}\right\}_{i}$. From this we infer

$$
\begin{aligned}
& \left|R\left(\nabla_{Z}^{i_{1}} z, \nabla_{Z}^{i_{2}} c^{\prime}\right) \nabla_{Z}^{i_{3}} \nabla_{s} \nabla_{Z}^{u-i} J\right| \\
& \leq C^{\prime} Q_{i_{2}}^{(2)}\left(\left(\left|Y_{1}\right|+\left|Y_{2}-Y_{1}\right|\right), \cdots,\left(\left|\nabla^{i_{2}} Y_{1}\right|+\left|\nabla^{i_{2}}\left(Y_{2}-Y_{1}\right)\right|\right)\right) \\
& \cdot\left\{C\left(\left|Y_{1}\right|+\left|Y_{2}-Y_{1}\right|\right) Q_{u-i+i_{3}+1}^{(4)}\left(\left(\left|Y_{1}\right|+\left|Y_{2}-Y_{1}\right|\right), \cdots,\left|\nabla^{u-i+i_{3}+1}\left(Y_{2}-Y_{1}\right)\right|\right)\right. \\
& \left.+\sum_{j=1}^{i_{3}} \sum_{k_{1}^{(3)}+k_{2}^{(3)}+k_{3}^{(3)}=i_{3}-j} Q_{u-i+k_{1}^{(3)}}^{(4)} Q_{k_{2}^{(3)}}^{(2)} Q_{u-i+j-1+k_{3}^{(3)}}^{(4)}\right\} \\
& =Q_{i_{1}, i_{2}, i_{3}}^{(u-i, i-2)}\left(\left(\left|Y_{1}\right|+\left|Y_{2}-Y_{1}\right|\right), \cdots,\left(\left|\nabla^{u-2} Y_{1}\right|+\left|\nabla^{u-2}\left(Y_{2}-Y_{1}\right)\right|,\left|Y_{2}-Y_{1}\right|\right.\right. \\
& \left.\cdots,\left|\nabla^{u-2}\left(Y_{2}-Y_{1}\right)\right|\right),
\end{aligned}
$$

where $Q_{i_{1}, i_{2}, i_{3}}^{(u-i, 2)}$ is a polynomial in the indicated variables with positive coefficients independent of $t_{0}$ and such that each monomial contains a factor $\left(\left|\nabla^{i} Y_{1}\right|+\mid \nabla^{i}\left(Y_{2}-\right.\right.$ $\left.\left.Y_{1}\right) \mid\right)\left|\nabla^{j}\left(Y_{2}-Y_{1}\right)\right|$.

Summing up, we get

$$
\begin{aligned}
\left|\nabla_{Z}^{i-2} \mathscr{R}\left(Z, \nabla_{Z}^{u-i} J\right)\right| & =2\left|\nabla_{Z}^{i-2} R\left(Z, c^{\prime}\right) \nabla_{s} \nabla_{Z}^{u-i} J\right| \\
& \leq Q^{(u-i, i-2)}\left(\left(\left|Y_{1}\right|+\left|Y_{2}-Y_{1}\right|\right), \cdots,\left|\nabla^{u-2}\left(Y_{2}-Y_{1}\right)\right|\right) \\
& =\sum_{i_{1}+i_{2}+i_{3}=i-2} Q_{i_{1}, i_{2}, i_{3}}^{(u-i, i-2)}
\end{aligned}
$$


and

$$
\begin{aligned}
& \left.\mid \mathscr{R}\left(Z, \nabla_{Z}^{u-2} J\right)+\nabla_{Z} \mathscr{R}\left(Z, \nabla_{Z}^{u-3} J\right)+\cdots+\nabla_{Z}^{u-2} \mathscr{R}(Z, J)\right) \mid \\
& \leq Q^{(u-2)}\left(\left(\left|Y_{1}\right|+\left|\nabla\left(Y_{2}-Y_{1}\right)\right|\right), \cdots,\left|\nabla^{u-2}\left(Y_{2}-Y_{1}\right)\right|\right) \\
& \equiv \sum_{i=1}^{u-2} Q^{(u-i, i-2)}
\end{aligned}
$$

where $Q^{u-2}$ is a polynomial in the indicated variables with positive coefficients independent of $t_{0}$ and such that each monomial contains a factor $\left(\left|\nabla^{i} Y_{1}\right|+\mid \nabla^{i}\left(Y_{2}-\right.\right.$ $\left.\left.Y_{1}\right) \mid\right)\left|\nabla^{j}\left(Y_{2}-Y_{1}\right)\right|$.

The remaining terms in $(7.92)$ are $-\nabla_{Z}^{u} \nabla_{s} P_{s} J^{\prime}(0)=0$ and $s R\left(\nabla_{Z}^{u} J(s), c^{\prime}\right) c^{\prime}$.

$$
\begin{aligned}
\left|R\left(\nabla_{Z}^{u} J(s), c^{\prime}\right) c^{\prime}\right| & \leq C^{\prime}\left(\left|Y_{1}\right|+\left|Y_{2}-Y_{1}\right|\right)^{2}\left|\nabla_{Z}^{u} J(s)\right| \\
& \leq C\left(\left|Y_{1}\right|+\left|Y_{2}-Y_{1}\right|\right)^{2} Q_{u}^{(4)}\left(\left(\left|Y_{1}\right|+\left|Y_{2}-Y_{1}\right|\right), \cdots,\left|\nabla^{u}\left(Y_{2}-Y_{1}\right)\right|\right) .
\end{aligned}
$$

We add up all our estimates:

$$
\begin{aligned}
& \mid-(1+s) \sum_{i=1}^{u} \nabla_{Z}^{u-i} R\left(\left(J(s), c^{\prime}\right) \nabla_{Z}^{i-1} J(s)+\sum_{i=1}^{u} \nabla_{Z}^{u-i} R\left(P_{s} J^{\prime}(0), c^{\prime}\right) \nabla_{Z}^{i-1} P_{s} J^{\prime}(0)\right. \\
& -\nabla_{Z}^{u} \nabla_{s} P_{s} J^{\prime}(0)+s R\left(\nabla_{Z}^{u} J(s), c^{\prime}\right) c^{\prime}+\mathscr{R}\left(Z, \nabla_{Z}^{u-2} J\right)+\cdots+\nabla_{Z}^{u-2} \mathscr{R}(Z, J) \mid \\
& =\mid-s \sum_{i=1}^{u} \nabla_{Z}^{u-i} R\left(\left(J(s), c^{\prime}\right) \nabla_{Z}^{i-1} J(s)+\sum_{i=1}^{u} \nabla_{Z}^{u-i} R\left(P_{s} J^{\prime}(0)-J(s), c^{\prime}\right) \nabla_{Z}^{i-1} P_{s} J^{\prime}(0)\right. \\
& +\sum_{i=1}^{u} \nabla_{Z}^{u-i} R\left(J(s), c^{\prime}\right) \nabla_{Z}^{i-1} P_{s}\left(J^{\prime}(0)-J(s)\right)+s R\left(\nabla_{Z}^{u} J(s), c^{\prime}\right) c^{\prime} \\
& +2 \sum_{i=2}^{u} \nabla_{Z}^{i-2} R\left(\left(Z, c^{\prime}\right) \nabla_{s} \nabla_{Z}^{u-i} J \mid\right. \\
& \leq C\left[\sum_{i=1}^{u} \sum_{i_{1}+i_{2}+i_{3}=u-i} Q_{i_{1}}^{(4)} Q_{i_{2}}^{(2)} Q_{i-1+i_{3}}^{(4)}\right. \\
& +\sum_{i=1}^{u} \sum_{i_{1}+i_{2}+i_{3}=u-i} Q_{i_{1}}^{(1)} Q_{i_{2}}^{(2)} Q_{i-1+i_{3}}^{(3)} \\
& +\sum_{i=1}^{u} \sum_{i_{1}+i_{2}+i_{3}=u-i} Q_{i_{1}}^{(4)} Q_{i_{2}}^{(2)} Q_{i-1+i_{3}}^{(1)} \\
& +Q_{u}^{(4)}\left(\left|Y_{1}\right|+\left|Y_{2}-Y_{1}\right|\right)^{2} \\
& \left.+Q^{(u-2)}\right] \\
& =p^{(u)}\left(\left(\left|Y_{1}\right|+\left|Y_{2}-Y_{1}\right|\right), \cdots,\left(\left|\nabla^{u} Y_{1}+\right| \nabla^{u}\left(Y_{2}-Y_{1}\right) \mid\right),\left|Y_{2}-Y_{1}\right|,\right. \\
& \left.\cdots,\left|\nabla^{u}\left(Y_{2}-Y_{1}\right)\right|\right),
\end{aligned}
$$

where $p^{(u)}$ is a polynomial in the indicated variables with positive coefficients independent of $t_{0}$ and such that each monomial contains a factor $\left(\left|\nabla^{i} Y_{1}\right|+\mid \nabla^{i}\left(Y_{2}-\right.\right.$ $\left.\left.Y_{1}\right) \mid\right)\left|\nabla^{j}\left(Y_{2}-Y_{1}\right)\right|$. The latter follows from the fact that in (7.112)-(7.116) $Q_{i}^{(4)}$ (which contains monomials $\left.\left|\nabla^{k}\left(Y_{2}-Y_{1}\right)\right|\right)$ always appears in connection with $Q_{j}^{2}$ or powers of $\left(\left|\nabla^{t} Y_{1}+\right| \nabla^{t}\left(Y_{2}-Y_{1}\right) \mid\right)$. 
We turn to equation (7.92),

$$
\begin{aligned}
\mid \nabla_{Z}^{u}\left(J(s)-\nabla_{Z}^{u} P_{s} J^{\prime}(0)-\left.s \nabla_{Z}^{u} J^{\prime}(s)\right|^{\prime}\right. & \leq \mid\left[\nabla_{Z}^{u}\left(J(s)-\nabla_{Z}^{u} P_{s} J^{\prime}(0)-s \nabla_{Z}^{u} J^{\prime}(s)\right]^{\prime} \mid\right. \\
& \leq p^{(u)}\left(\left(\left|Y_{1}\right|+\left|Y_{2}-Y_{1}\right|\right), \cdots,\left|\nabla^{u}\left(Y_{2}-Y_{1}\right)\right|\right),
\end{aligned}
$$

from

$$
\mid \nabla_{Z}^{u}\left(J(1)-\nabla_{Z}^{u} P_{1} J^{\prime}(0)-\nabla_{Z}^{u} J^{\prime}(1)|-| \nabla_{Z}^{u} J^{\prime}(0) \mid \leq \int_{0}^{1} p^{(u)} d s,\right.
$$

we conclude that

$$
\begin{aligned}
\mid \nabla_{Z}^{u}\left(J(1)-\nabla_{Z}^{u} P_{1} J^{\prime}(0) \mid \leq\right. & \left|\nabla_{Z}^{u} J^{\prime}(0)\right|+\left|\nabla_{Z}^{u} J^{\prime}(1)\right|+p^{(u)} \\
\leq & 2 Q_{u}^{(4)}\left(\left(\left|Y_{1}\right|+\left|Y_{2}-Y_{1}\right|\right), \cdots,\left|\nabla^{u}\left(Y_{2}-Y_{1}\right)\right|\right)+p^{(u)} \\
= & Q_{u}\left(\left(\left|Y_{1}\right|+\left|Y_{2}-Y_{1}\right|\right), \cdots,\left(\left|\nabla^{u} Y_{1}\right|+\left|\nabla^{u}\left(Y_{2}-Y_{1}\right)\right|\right),\right. \\
& \left.\left|Y_{2}-Y_{1}\right|, \cdots,\left|\nabla^{u}\left(Y_{2}-Y_{1}\right)\right|\right),
\end{aligned}
$$

where $Q_{u}$ is a polynomial in the indicated variables with positive coefficients independent of $t_{0}$ without constant term and such that each term contains $\left|\nabla^{j}\left(Y_{2}-Y_{1}\right)\right|$ as a factor.

(7.118) accomplishes our induction proof of the estimate of $\left|\nabla^{u}\left(J(1)-P_{1} J^{\prime}(0)\right)\right|$.

But our task was to estimate

$$
\nabla^{u} P_{-Y_{\theta}} \nabla_{c^{\prime}} \Delta_{\exp Y_{\theta}}\left(J(1)-P_{1}\left(Y_{2}-Y_{1}\right)\right),
$$

which reduces to the estimate of

$$
\begin{aligned}
& \nabla^{u} \nabla_{c^{\prime}} g_{0}^{i j} \nabla_{j} \nabla_{i}\left(J(1)-P_{1}\left(Y_{2}-Y_{1}\right)\right) \\
& =g_{0}^{i j} \nabla^{u} \nabla_{c^{\prime}} \nabla_{j} \nabla_{i}\left(J(1)-P_{1}\left(Y_{2}-Y_{1}\right)\right) \\
& =\nabla_{c^{\prime}} \nabla^{u}\left(J(1)-P_{1}\left(Y_{2}-Y_{1}\right)\right) \\
& +\sum_{i=1}^{u} \nabla^{u-i} R\left(J(1)-P_{1}\left(Y_{2}-Y_{1}\right), c^{\prime}\right) \nabla^{i-1}\left(J(1)-P_{1}\left(Y_{2}-Y_{1}\right)\right),
\end{aligned}
$$

as we have seen in (7.91).

The first term on the RHS of (7.119) can be estimated as

$$
\begin{aligned}
& \left|\nabla_{c^{\prime}} \nabla^{u}\left(J(1)-P_{1}\left(Y_{2}-Y_{1}\right)\right)\right| \\
\leq & \left|c^{\prime}\right|\left|\nabla^{u+1}\left(J(1)-P_{1}\left(Y_{2}-Y_{1}\right)\right)\right| \\
\leq & C\left(\left|Y_{1}\right|+\left|Y_{2}-Y_{1}\right|\right) Q_{u+1} \\
= & P_{u}\left(\left(\left|Y_{1}\right|+\left|Y_{2}-Y_{1}\right|\right), \cdots,\left(\left|\nabla^{u+1} Y_{1}+\right| \nabla^{u+1}\left(Y_{2}-Y_{1}\right) \mid\right),\right. \\
& \left.\left|Y_{2}-Y_{1}\right|, \cdots,\left|\nabla^{u+1}\left(Y_{2}-Y_{1}\right)\right|\right),
\end{aligned}
$$

where $P_{u}$ is a polynomial in the indicated variables with positive coefficients independent of $t_{0}$ and such that each monomial contains a factor $\left(\left|\nabla^{i} Y_{1}\right|+\mid \nabla^{i}\left(Y_{2}-\right.\right.$ $\left.\left.Y_{1}\right) \mid\right)\left|\nabla^{j}\left(Y_{2}-Y_{1}\right)\right|$.

Since all norm products stem from the Leibniz rule, they are in $L_{2}$ according to the module structure theorem, and we obtain from the latter theorem

$$
\begin{aligned}
\left|\nabla_{c^{\prime}} \nabla^{u}\left(J(1)-P_{1}\left(Y_{2}-Y_{1}\right)\right)\right|_{L_{2}} \leq & C\left(\left|Y_{1}\right|_{L_{2}}+\left|Y_{2}-Y_{1}\right|_{L_{2}}\right)\left|Q_{u+1}\right|_{L_{2}} \\
\leq & C\left(\left|Y_{1}\right|_{L_{2}}+\left|Y_{2}-Y_{1}\right|_{L_{2}}\right) Q_{u+1}\left(\left|Y_{1}\right|_{r}+\left|Y_{2}-Y_{1}\right|_{r}\right. \\
& \left.\left|Y_{2}-Y_{1}\right|_{r}\right)
\end{aligned}
$$


where $Q_{u+1}$ is a polynomial in the indicated variables with positive coefficients independent of $t_{0}$ and such that each monomial contains $\left|Y_{2}-Y_{1}\right|_{r}$ as a factor.

Hence

$$
\left|\nabla_{c^{\prime}} \nabla^{u}\left(J(1)-P_{1}\left(Y_{2}-Y_{1}\right)\right)\right|_{L_{2}} \leq \tilde{Q}_{u+1}\left|Y_{2}-Y_{1}\right|_{r}
$$

where $\tilde{Q}_{u+1}=C\left(\left|Y_{1}\right|_{L_{2}}+\left|Y_{2}-Y_{1}\right|_{L_{2}}\right) Q_{u+1} /\left|Y_{2}-Y_{1}\right|_{r}$ has no constant term. If $u$ runs from 0 to $r-2$, we obtain

$$
\left|P_{-Y_{\theta}} \nabla_{c^{\prime}} \Delta_{\exp Y_{\theta}}\left(J(1)-P_{1}\left(Y_{2}-Y_{1}\right)\right)\right|_{r-2} \leq \tilde{p}_{r}\left|Y_{2}-Y_{1}\right|_{r}
$$

where $\tilde{p}_{r}=\tilde{p}_{r}\left(\left|Y_{1}\right|_{r}+\left|Y_{2}-Y_{1}\right|_{r},\left|Y_{2}-Y_{1}\right|_{r}\right)$ is a polynomial in the indicated variables with positive coefficients independent of $t_{0}$ and has no constant term, in particular,

$$
\left|P_{-Y_{\theta}} \nabla_{c^{\prime}} \Delta_{\exp Y_{\theta}}\left(J(1)-P_{1}\left(Y_{2}-Y_{1}\right)\right)\right|_{r-2}=o(1)\left|Y_{2}-Y_{1}\right|_{r},
$$

$o(1) \rightarrow 0\left(\left|Y_{1}\right|_{r},\left|Y_{2}\right|_{r} \rightarrow 0\right)$ with rate independent of $t_{0}$.

$|(7.65)|_{r-2}$ and (7.66) are completely estimated,

$$
\begin{aligned}
& \left|-\Delta_{f_{t_{0}}}\left(Y_{2}-Y_{1}\right)+P_{-Y_{\theta}} \Delta_{\exp Y_{\theta}} c_{Y_{\theta}}^{\prime}\right|_{r-2} \\
& \leq\left|P_{-Y_{\theta}} \Delta_{\exp Y_{\theta}} P_{Y_{\theta}}\left(Y_{2}-Y_{1}\right)-\Delta_{f_{t_{0}}}\left(Y_{2}-Y_{1}\right)\right|_{r-2} \\
& +\left|P_{-Y_{\theta}} \nabla_{c^{\prime}} \Delta_{\exp Y_{\theta}}\left(J(1)-P_{1}\left(Y_{2}-Y_{1}\right)\right)\right|_{r-2} \\
& =o(1)\left|Y_{2}-Y_{1}\right|_{r} .
\end{aligned}
$$

The remaining expressions are

$$
\begin{gathered}
t r_{g_{0}} R^{g_{t_{0}}}\left(Y_{2}-Y_{1}, d f_{t_{0}}\right) d f_{t_{0}}-P_{-Y_{\theta}} t r_{g_{0}} R^{g_{0}}\left(c_{Y_{\theta}}^{\prime}, d\left(\exp Y_{\theta}\right)\right) d\left(\exp Y_{\theta}\right) \\
-P_{-Y_{\theta}} g_{0}^{i j} \nabla_{c_{Y_{\theta}}^{\prime}}\left(\nabla^{g_{t}}-\nabla^{g_{0}}\right)_{j} d\left(\exp Y_{\theta}\right)\left(e_{i}\right) .
\end{gathered}
$$

We write (7.125) as

$t r_{g_{0}} R^{g_{t_{0}}}\left(Y_{2}-Y_{1}, d f_{t_{0}}\right) d f_{t_{0}}-P_{-Y_{\theta}} \operatorname{tr}_{g_{0}} R^{g_{t_{0}}}\left(d\left(\exp Y_{\theta}\right)\left(Y_{2}-Y_{1}\right), d\left(\exp Y_{\theta}\right)\right) d\left(\exp Y_{\theta}\right) \quad$ (7.127) $+P_{-Y_{\theta}} t r_{g_{0}} R^{g_{t_{0}}}\left(d\left(\exp Y_{\theta}\right)\left(Y_{2}-Y_{1}\right), d\left(\exp Y_{\theta}\right)\right) d\left(\exp Y_{\theta}\right)-P_{-Y_{\theta}} t r_{g_{0}} R^{g_{0}}\left(d\left(\exp Y_{\theta}\right)\left(Y_{2}-Y_{1}\right)\right.$, $\left.d\left(\exp Y_{\theta}\right)\right) d\left(\exp Y_{\theta}\right)$

and express (7.127) in local coordinates as

$$
\begin{gathered}
-\int_{0}^{1} \frac{d}{d s}\left[P_{-s Y_{\theta}} t r_{g_{0}} R^{g_{t_{0}}}\left(d\left(\exp s Y_{\theta}\right)\left(Y_{2}-Y_{1}\right), d\left(\exp s Y_{\theta}\right)\right) d\left(\exp s Y_{\theta}\right)\right] d s \\
=-\int_{0}^{1} P_{-s Y_{\theta}} \nabla_{c^{\prime}} t r_{g_{0}} R^{g_{t_{0}}}\left(d\left(\exp s Y_{\theta}\right)\left(Y_{2}-Y_{1}\right), d\left(\exp s Y_{\theta}\right)\right) d\left(\exp s Y_{\theta}\right) d s \\
\quad \operatorname{tr}_{g_{0}} \nabla_{c^{\prime}} R^{g_{t_{0}}}\left(d\left(\exp s Y_{\theta}\right)\left(Y_{2}-Y_{1}\right), d\left(\exp s Y_{\theta}\right)\right) d\left(\exp s Y_{\theta}\right) \\
=t r_{g_{0}}\left(\nabla_{c^{\prime}} R^{g_{t_{0}}}\right)\left(d\left(\exp s Y_{\theta}\right)\left(Y_{2}-Y_{1}\right), d\left(\exp s Y_{\theta}\right)\right) d\left(\exp s Y_{\theta}\right) \\
+\operatorname{tr}_{g_{0}} R^{g_{t_{0}}}\left(\nabla_{c^{\prime}}\left(d\left(\exp s Y_{\theta}\right)\left(Y_{2}-Y_{1}\right)\right), d\left(\exp s Y_{\theta}\right)\right) d\left(\exp s Y_{\theta}\right)
\end{gathered}
$$




$$
\begin{aligned}
& +t r_{g_{0}} R^{g_{t_{0}}}\left(d\left(\exp s Y_{\theta}\right)\left(Y_{2}-Y_{1}\right), \nabla_{c^{\prime}}\left(d\left(\exp s Y_{\theta}\right)\right)\right) d\left(\exp s Y_{\theta}\right) \\
& \quad+t r_{g_{0}} R^{g_{t_{0}}}\left(d\left(\exp s Y_{\theta}\right)\left(Y_{2}-Y_{1}\right), d\left(\exp s Y_{\theta}\right) \nabla_{c^{\prime}}\left(d\left(\exp s Y_{\theta}\right)\right) .\right.
\end{aligned}
$$

Since

$$
\begin{aligned}
\mid d\left(\exp s Y_{\theta}\right) & \left(Y_{2}-Y_{1}\right)|=| \frac{J(s)}{s}|\leq C| Y_{2}-Y_{1} \mid, \\
\left|\nabla_{c^{\prime}} R^{g_{t_{0}}}\right| & \leq\left|c^{\prime}\right|\left|\nabla R^{g_{t_{0}}}\right| \\
& =\left|c^{\prime}\right|\left|\nabla^{g_{t_{0}}} R^{g_{t_{0}}}-\left(\nabla^{g_{t_{0}}}-\nabla\right) R^{g_{t_{0}}}\right| \\
& =\left|c^{\prime}\right|\left|\nabla^{g_{t_{0}}}-\nabla\right|\left|R^{g_{t_{0}}}\right| \\
& \leq C_{2}\left(\left|Y_{1}\right|+\left|Y_{2}-Y_{1}\right|\right)\left|\nabla^{g_{t_{0}}}-\nabla\right|,
\end{aligned}
$$

hence,

$$
(7.129) \leq C\left|\nabla^{g_{t_{0}}}-\nabla\right|\left(\left|Y_{1}\right|+\left|Y_{2}-Y_{1}\right|\right)\left|Y_{2}-Y_{1}\right| .
$$

For the estimate of (7.130), we use

$$
\nabla\left[d\left(\exp s Y_{\theta}\right)\left(Y_{2}-Y_{1}\right)\right]=\nabla\left(d\left(\exp s Y_{\theta}\right)\right)\left(Y_{2}-Y_{1}\right)+d\left(\exp s Y_{\theta}\right)\left(\nabla\left(Y_{2}-Y_{1}\right)\right)
$$

and

$$
\left|\nabla^{u}(\operatorname{dexp} Y)\right| \leq P_{u}\left(|Y|,|\nabla Y|, \cdots,\left|\nabla^{u} Y\right|\right), \quad 1 \leq u \leq r,
$$

and obtain

$$
\begin{gathered}
(7.130) \leq C\left(\left|Y_{1}\right|+\left|Y_{2}-Y_{1}\right|\right)\left[P _ { 1 } \left(\left(\left|Y_{1}\right|+\left|Y_{2}-Y_{1}\right|\right),\right.\right. \\
\left.\left(\left|\nabla Y_{1}\right|+\left|\nabla\left(Y_{2}-Y_{1}\right)\right|\right)\left|Y_{2}-Y_{1}\right|+\left|\nabla\left(Y_{2}-Y_{1}\right)\right|\right] \\
(7.131) \leq C\left|Y_{2}-Y_{1}\right|\left(\left|Y_{1}\right|+\left|Y_{2}-Y_{1}\right|\right)\left|Y_{2}-Y_{1}\right| P_{1} \\
(7.132) \leq C\left|Y_{2}-Y_{1}\right|\left(\left|Y_{1}\right|+\left|Y_{2}-Y_{1}\right|\right)\left|Y_{2}-Y_{1}\right| P_{1}
\end{gathered}
$$

where $P_{u}$ has no constant term.

Next we must estimate

$$
\nabla^{u}(7.128)=\nabla^{u}(7.129)+\cdots+\nabla^{u}(7.132)
$$

and we perform induction. Suppose

$\left|\nabla^{i}(7.128)\right| \leq P_{i}\left(\left(\left|Y_{1}\right|+\left|Y_{2}-Y_{1}\right|\right), \cdots,\left(\left|\nabla Y_{1}\right|+\left|\nabla\left(Y_{2}-Y_{1}\right)\right|\right),\left|Y_{2}-Y_{1}\right|,\left|\nabla\left(Y_{2}-Y_{1}\right)\right|\right)$

for $0 \leq i \leq u-1$, where $P_{i}$ is a polynomial in the indicated variables with positive coefficients independent of $t_{0}$ and such that each monomial contains a factor $\left(\left|\nabla^{i} Y_{1}\right|+\right.$ $\left.\left|\nabla^{i}\left(Y_{2}-Y_{1}\right)\right|\right)\left|\nabla^{j}\left(Y_{2}-Y_{1}\right)\right|$. We admit additionally Sobolev functions as factors. For $i=0$, this follows from (7.134)-(7.137). 
We use again

$$
\begin{aligned}
\left|\nabla_{Z}^{u} \operatorname{tr}_{g_{0}} \nabla_{c^{\prime}} X\right| & =\operatorname{tr}_{g_{0}} \nabla_{Z}^{u} \nabla_{c^{\prime}} X \mid \\
& =\left|\operatorname{tr}_{g_{0}}\left(\nabla_{c^{\prime}} \nabla_{Z}^{u} X+\sum_{i=1}^{u} \nabla_{Z}^{u-i} R\left(X, c^{\prime}\right) \nabla_{Z}^{i-1} X\right)\right| \\
& \leq C\left[\left|c^{\prime}\right|\left|\nabla^{u+1} X\right|+\sum_{i=1}^{u} \sum_{i_{1}+i_{2}+i_{3}=u-i}\left|\nabla^{i_{1}} X\right|\left|\nabla^{i_{2}} c^{\prime}\right|\left|\nabla^{i_{3}} X\right|\right] .(7.1
\end{aligned}
$$

In our case

$$
X=R^{g_{t_{0}}}\left(\left(\operatorname{dexp} s Y_{\theta}\right)\left(Y_{2}-Y_{1}\right),\left(\operatorname{dexp} s Y_{\theta}\right)\left(e_{i}\right)\right)\left(\operatorname{dexp} s Y_{\theta}\right)\left(e_{i}\right) .
$$

By the case $i=0$ and our induction assumption, the term

$$
\sum_{i=1}^{u} \sum_{i_{1}+i_{2}+i_{3}=u-i}\left|\nabla^{i_{1}} X\right|\left|\nabla^{i_{2}} c^{\prime}\right|\left|\nabla^{i_{3}} X\right|
$$

has already the structure of such a polynomial. In $\left|c^{\prime}\right|\left|\nabla^{u+1} X\right|,\left|c^{\prime}\right| \leq \sqrt{2}\left(\left|Y_{1}\right|+\mid Y_{2}-\right.$ $\left.Y_{1} \mid\right)$ already generates a required factor. There remains to estimate

$$
\begin{aligned}
& \nabla^{u} R^{g_{t_{0}}}\left(\left(d \exp s Y_{\theta}\right)\left(Y_{2}-Y_{1}\right),\left(d \exp s Y_{\theta}\right)\left(e_{i}\right)\right)\left(\operatorname{dexp} s Y_{\theta}\right)\left(e_{i}\right) \\
& =\sum_{i_{1}+i_{2}+i_{3}+i_{4}=u}\left(\nabla^{i_{1}} R^{g_{t_{0}}}\right)\left(\nabla^{i_{2}}\left[\left(\operatorname{dexp} s Y_{\theta}\right)\left(Y_{2}-Y_{1}\right)\right],\right. \\
& \left.\nabla^{i_{3}}\left[\left(\operatorname{dexp} s Y_{\theta}\right)\left(e_{i}\right)\right]\right) \nabla^{i_{4}}\left[\left(\operatorname{dexp} s Y_{\theta}\right)\left(e_{i}\right)\right] .
\end{aligned}
$$

Quite parallel to (7.134), we get

$$
\left|\nabla^{i_{1}} R^{g_{t_{0}}}\right| \leq C\left|\nabla^{i_{1}}\left(\nabla^{g_{t_{0}}}-\nabla\right)\right|
$$

which is in $L_{2}$.

For $i_{1}>1$,

$$
\begin{aligned}
& \left|\nabla^{i_{2}}\left[\left(d \exp s Y_{\theta}\right)\left(Y_{2}-Y_{1}\right)\right]\right| \\
\leq & \left\{\begin{array}{c}
C\left|Y_{2}-Y_{1}\right|, \quad i_{2}=0, \\
C P_{j_{1}}\left(\left(\left|Y_{1}\right|+\left|Y_{2}-Y_{1}\right|\right), \cdots,\left(\left|\nabla^{j_{2}} Y_{1}\right|+\left|\nabla^{j_{2}}\left(Y_{2}-Y_{1}\right)\right|\right)\right. \\
\cdot\left|\nabla^{j_{2}}\left(Y_{2}-Y_{1}\right)\right|, \quad j_{1}+j_{2}=i_{2}>0 .
\end{array}\right. \\
\mid & \nabla^{i}\left[\left(\operatorname{dexp} s Y_{\theta}\right)\left(e_{i}\right)\right] \mid \\
\leq & \left\{\begin{array}{c}
C, \quad i=0, \\
C P_{i}\left(\left(\left|Y_{1}\right|+\left|Y_{2}-Y_{1}\right|\right), \cdots,\left(\left|\nabla^{i} Y_{1}\right|+\left|\nabla^{i}\left(Y_{2}-Y_{1}\right)\right|\right), \quad i \geq 1 .\right.
\end{array}\right.
\end{aligned}
$$

We see from (7.140)-(7.142) that (7.139) can be estimated by a sum of polynomials in $\left|Y_{1}\right|+\left|Y_{2}-Y_{1}\right|,\left|\nabla Y_{1}\right|+\left|\nabla\left(Y_{2}-Y_{1}\right)\right|, \cdots,\left|Y_{2}-Y_{1}\right|,\left|\nabla\left(Y_{2}-Y_{1}\right)\right|$, for $i_{1}>1$, the corresponding polynomials have an $\Omega^{2, r-2}$-function as factor, each polynomial has some $\left|\nabla^{j}\left(Y_{2}-Y_{1}\right)\right|$ as factor, but there are terms without $\left|\nabla^{j} Y_{1}\right|+\left|\nabla^{j}\left(Y_{2}-Y_{1}\right)\right|-$ factor $\left(i_{2}, i_{3}, i_{4}=0\right)$. This missing factor is covered by $\left|c^{\prime}\right| \leq \sqrt{2}\left(\left|Y_{1}\right|+\left|Y_{2}-Y_{1}\right|\right)$ in $\left|c^{\prime}\right|\left|\nabla^{u+1} X\right|$. All expressions in the estimate of $\left|\nabla^{u}(7.128)\right|$ stem from the Leibniz rule, and we can apply the module structure theorem. 
Finally, we have to estimate

$$
\begin{aligned}
& \mid \nabla^{u} P_{-s Y_{\theta}} t r_{g_{0}} R^{g_{0}}\left(d\left(\exp s Y_{\theta}\right)\left(Y_{2}-Y_{1}\right), d\left(\exp s Y_{\theta}\right)\left(d\left(\exp s Y_{\theta}\right)\right) \equiv\left|\nabla^{u} P X\right|\right. \\
& =\left|\sum_{i_{1}+i_{2}=u}\left(\nabla^{i_{1}} P\right)\left(\nabla^{i_{2}} X\right)\right| \\
& \leq \sum_{i_{1}+i_{2}=u}\left|\nabla^{i_{1}} P\right|\left|\nabla^{i_{2}} X\right| .
\end{aligned}
$$

According to $(7.26),(7.27)$, for $i_{1} \leq 2,\left|\nabla^{i_{1}} P\right|$ can be estimated by a constant, for $i_{1}>2$, it is an $\Omega^{2, r-2}$-function.

We obtain from (7.143) and the preceding estimates (7.133)-(7.142) that

$\left|\operatorname{tr}_{g_{0}} R^{g_{t_{0}}}\left(Y_{2}-Y_{1}, d f_{t_{0}}\right) d f_{t_{0}}-P_{-Y_{\theta}} t r_{g_{0}} R^{g_{t_{0}}}\left(d\left(\exp Y_{\theta}\right)\left(Y_{2}-Y_{1}\right), d\left(\exp Y_{\theta}\right)\right) d\left(\exp Y_{\theta}\right)\right|_{r-2}$ $\leq P_{r}\left(\left|Y_{1}\right|_{r}+\left|Y_{2}-Y_{1}\right|_{r},\left|Y_{2}-Y_{1}\right|_{r}\right)\left|Y_{2}-Y_{1}\right|_{r}$ $\leq o(1)\left|Y_{2}-Y_{1}\right|_{r}$,

where $o(1) \rightarrow 0$ as $\left|Y_{1}\right|_{r},\left|Y_{2}\right|_{r} \rightarrow 0$, and $p_{r}$ is a polynomial in the indicated variables with positive coefficients independent of $t_{0}$ and without constant term.

We still have to estimate

$$
\begin{aligned}
& P_{-Y_{\theta}} t_{g_{0}} R^{g_{t_{0}}}\left(d\left(\exp Y_{\theta}\right)\left(Y_{2}-Y_{1}\right), d\left(\exp Y_{\theta}\right)\right) d\left(\exp Y_{\theta}\right) \\
& -P_{-Y_{\theta}} \operatorname{tr}_{g_{0}} R^{g_{0}}\left(d\left(\exp Y_{\theta}\right)\left(Y_{2}-Y_{1}\right), d\left(\exp Y_{\theta}\right)\right) d\left(\exp Y_{\theta}\right)
\end{aligned}
$$

and

$$
P_{-Y_{\theta}} g_{0}^{i j} \nabla_{c_{Y_{\theta}}^{\prime}}\left(\nabla^{g_{t}}-\nabla^{g_{0}}\right)_{j}\left(d\left(\exp Y_{\theta}\right)\right)\left(e_{i}\right) .
$$

We start with (7.145) and write in normal coordinates (w.r.t. $g_{0}$ )

$$
\begin{aligned}
(7.145)= & \int_{0}^{1} \frac{d}{d s}\left[P_{-s Y_{\theta}} t r_{g_{0}}\left(R^{g_{s t_{0}}}-R^{g_{0}}\right)\left(d\left(\exp Y_{\theta}\right)\left(Y_{2}-Y_{1}\right), d\left(\exp Y_{\theta}\right)\right) d\left(\exp Y_{\theta}\right)\right] d s \\
= & \int_{0}^{1}\left[P _ { - s Y _ { \theta } } \nabla _ { c ^ { \prime } } t r _ { g _ { 0 } } ( R ^ { g _ { s t _ { 0 } } } - R ^ { g _ { 0 } } ) \left(d\left(\exp Y_{\theta}\right)\left(Y_{2}-Y_{1}\right)\right.\right. \\
& \left.\left.d\left(\exp Y_{\theta}\right)\right) d\left(\exp Y_{\theta}\right)\right] d s \\
& \left|P_{-s Y_{\theta}} \nabla_{c^{\prime}} t r_{g_{0}}\left(R^{g_{s t_{0}}}-R^{g_{0}}\right)\left(d\left(\exp Y_{\theta}\right)\left(Y_{2}-Y_{1}\right), d\left(\exp Y_{\theta}\right)\right) d\left(\exp Y_{\theta}\right)\right| \\
= & \left|t r_{g_{0}} \nabla_{c^{\prime}}\left(R^{g_{s t_{0}}}-R^{g_{0}}\right)\left(d\left(\exp Y_{\theta}\right)\left(Y_{2}-Y_{1}\right), d\left(\exp Y_{\theta}\right)\right) d\left(\exp Y_{\theta}\right)\right| \\
\leq & C_{0}\left|c^{\prime}\right|\left[\left|\nabla-\nabla^{g_{s t_{0}}}\right|\left|R^{g_{s t_{0}}}\right| g_{g_{0}}\left|Y_{2}-Y_{1}\right|\right. \\
\leq & C\left(\left|Y_{1}\right|+\left|Y_{2}-Y_{1}\right|\right)\left|Y_{2}-Y_{1}\right|
\end{aligned}
$$

where we used

$$
\begin{gathered}
\nabla\left(R^{g_{s t_{0}}}-R^{g_{0}}\right)=\nabla R^{g_{s t_{0}}}=\left[\left(\nabla-\nabla^{g_{s t_{0}}}\right)+\nabla^{g_{s t_{0}}}\right] R^{g_{s t_{0}}}=\left(\nabla-\nabla^{g_{s t_{0}}}\right) R^{g_{s t_{0}}}, \\
\nabla^{u} \nabla_{c^{\prime}} \operatorname{tr}_{g_{0}}\left(R^{g_{s t_{0}}}-R^{g_{0}}\right)\left(d\left(\exp s Y_{\theta}\right)\left(Y_{2}-Y_{1}\right), d\left(\exp s Y_{\theta}\right)\right) d\left(\exp s Y_{\theta}\right) \\
=\operatorname{tr}_{g_{0}}\left[\nabla_{c^{\prime}} \nabla^{u}\left(R^{g_{s t_{0}}}-R^{g_{0}}\right)\left(d\left(\exp s Y_{\theta}\right)\left(Y_{2}-Y_{1}\right), d\left(\exp s Y_{\theta}\right)\right) d\left(\exp s Y_{\theta}\right)\right.
\end{gathered}
$$




$$
\begin{aligned}
& +\sum_{i=1}^{u} \nabla^{u-i}\left(\left(R^{g_{s t_{0}}}-R^{g_{0}}\right)\left(d\left(\exp s Y_{\theta}\right)\left(Y_{2}-Y_{1}\right), d\left(\exp s Y_{\theta}\right)\right) d\left(\exp s Y_{\theta}\right),, c^{\prime}\right) \\
& \nabla^{i-1}\left(\left(R^{g_{s t_{0}}}-R^{g_{0}}\right)\left(d\left(\exp s Y_{\theta}\right)\left(Y_{2}-Y_{1}\right), d\left(\exp s Y_{\theta}\right)\right) d\left(\exp s Y_{\theta}\right)\right]
\end{aligned}
$$

We estimate (7.149) as above,

$$
\begin{aligned}
|(7.149)| \leq & C\left|c^{\prime}\right| \sum_{i_{1}+\cdots+i_{4}=u+1}\left|\nabla^{i_{1}} R^{g_{s t_{0}}}\right| \\
& \cdot\left|\nabla^{i_{2}}\left(d \exp s Y_{\theta}\right)\left(Y_{2}-Y_{1}\right)\right| \cdot\left|\nabla^{i_{3}}\left(\operatorname{dexp} s Y_{\theta}\right)\right| \cdot\left|\nabla^{i_{4}}\left(\operatorname{dexp} s Y_{\theta}\right)\right| .
\end{aligned}
$$

Here

$$
\begin{aligned}
&\left|\nabla^{i_{1}} R^{g_{s t_{0}}}\right| \leq\left\{\begin{array}{cl}
\text { const. }, & i_{1}=0 \\
L_{2}-\text { function, } & i_{1}>0,
\end{array}\right. \\
&\left|\nabla^{i_{2}}\left(\operatorname{dexp} s Y_{\theta}\right)\left(Y_{2}-Y_{1}\right)\right| \leq \sum_{j_{1}+j_{2}=i_{2}}\left|\nabla^{j_{1}} d \exp s Y_{\theta}\right|\left|\nabla^{j_{2}}\left(Y_{2}-Y_{1}\right)\right|,
\end{aligned}
$$

where

$$
\begin{aligned}
& \left|\nabla^{j_{1}} \operatorname{dexp} s Y_{\theta}\right| \\
& \leq\left\{\begin{array}{cc}
\text { const. }, & j_{1}=0 \\
P_{j_{1}}\left(\left(\left|Y_{1}\right|+\left|Y_{2}-Y_{1}\right|\right), \cdots,\left(\left|\nabla^{j_{1}} Y_{1}\right|+\left|\nabla^{j_{1}}\left(Y_{2}-Y_{1}\right)\right|\right),\right. & j_{1}>0 .
\end{array}\right.
\end{aligned}
$$

We then have that

$$
\begin{aligned}
|(7.149)| \leq & \tilde{Q}_{u}\left(\left(\left|Y_{1}\right|+\left|Y_{2}-Y_{1}\right|\right), \cdots,\left(\left|\nabla^{u+1} Y_{1}\right|+\left|\nabla^{u+1}\left(Y_{2}-Y_{1}\right)\right|\right),\right. \\
& \left.\left|Y_{2}-Y_{1}\right|, \cdots,\left|\nabla^{u+1}\left(Y_{2}-Y_{1}\right)\right|\right),
\end{aligned}
$$

where $\tilde{Q}_{u}$ is a polynomial in the indicated variables with positive coefficients (possibly Sobolev functions) independent of $t_{0}$ and such that each monomial contains a factor $\left|\nabla^{i} Y_{1}\right|+\left|\nabla^{i}\left(Y_{2}-Y_{1}\right)\right|\left|\nabla^{j}\left(Y_{2}-Y_{1}\right)\right|$ (the first factor is delivered already by $\left.\left|c^{\prime}\right|\right)$.

If we apply the inequalities (7.151)-(7.153) to (7.150), then we get quite analogous

$$
\begin{aligned}
|(7.150)| \leq & \tilde{Q}_{u-2}\left(\left(\left|Y_{1}\right|+\left|Y_{2}-Y_{1}\right|\right), \cdots,\left(\left|\nabla^{u-1} Y_{1}\right|+\left|\nabla^{u-1}\left(Y_{2}-Y_{1}\right)\right|\right),\right. \\
& \left.\left|Y_{2}-Y_{1}\right|, \cdots,\left|\nabla^{u-1}\left(Y_{2}-Y_{1}\right)\right|\right),
\end{aligned}
$$

where $\tilde{Q}_{u-2}$ has the same properties as $\tilde{Q}_{u}$ (the $c^{\prime}$ is contained in the curvature terms).

Altogether,

$$
\begin{aligned}
& \mid \nabla^{u} P_{-s Y_{\theta}} \nabla_{c^{\prime}} t r_{g_{0}}\left(R^{g_{t_{0}}}-R^{g_{0}}\right)\left(d\left(\exp Y_{\theta}\right)\left(Y_{2}-Y_{1}\right), d\left(\exp Y_{\theta}\right)\right) d\left(\exp Y_{\theta}\right) \\
& \leq \sum_{i_{1}+i_{2}=u+1}\left|\nabla^{i_{1}} P_{-s Y_{\theta}}\right|\left(\tilde{Q}_{i_{2}}+\tilde{Q}_{i_{2}-2}\right) \\
& =Q_{u}\left(\left(\left|Y_{1}\right|+\left|Y_{2}-Y_{1}\right|\right), \cdots,\left|\nabla^{u+1}\left(Y_{2}-Y_{1}\right)\right|\right) .
\end{aligned}
$$

All terms in the polynomial stem from the Leibniz rule. Summing up $u=0, \cdots, r-2$ and taking the $L_{2}$-norm, we obtain finally

$$
\begin{aligned}
& \left|P_{-Y_{\theta}} t_{g_{0}}\left(R^{g_{t_{0}}}-R^{g_{0}}\right)\left(d\left(\exp Y_{\theta}\right)\left(Y_{2}-Y_{1}\right), d\left(\exp Y_{\theta}\right)\right) d\left(\exp Y_{\theta}\right)\right|_{r-2} \\
& \leq Q_{r}\left(\left(\left|Y_{1}\right|_{r}+\left|Y_{2}-Y_{1}\right|_{r}\right),\left|Y_{2}-Y_{1}\right|_{r}\right) \\
& =P_{r}\left(\left(\left|Y_{1}\right|_{r}+\left|Y_{2}-Y_{1}\right|_{r}\right),\left|Y_{2}-Y_{1}\right|_{r}\right)\left|Y_{2}-Y_{1}\right|_{r} \\
& =o(1)\left|Y_{2}-Y_{1}\right|_{r}
\end{aligned}
$$


where $o(1) \rightarrow 0$ as $\left|Y_{1}\right|_{r},\left|Y_{2}\right|_{r} \rightarrow 0, P_{r}=Q_{r} /\left|Y_{2}-Y_{1}\right|_{r}$, and $Q_{r}\left(\left(\left|Y_{1}\right|_{r}+\left|Y_{2}-Y_{1}\right|_{r}\right), \mid Y_{2}-\right.$ $\left.\left.Y_{1}\right|_{r}\right)$ is a polynomial in the indicated variables with positive coefficients independent of $t_{0}$ and such that each monomial contains a factor $\left(\left|Y_{1}\right|_{r}+\left|Y_{2}-Y_{1}\right|_{r}\right)\left|Y_{2}-Y_{1}\right|_{r}$, which finishes the estimate of (7.125).

The last step in the estimate of (7.61), (7.62) is the estimate of

$$
\begin{aligned}
& P_{-Y_{\theta}} g_{0}^{i j} \nabla_{c_{Y_{\theta}^{\prime}}}\left(\nabla^{g_{t}}-\nabla^{g_{0}}\right)_{j} d\left(\exp Y_{\theta}\right)\left(e_{i}\right) \\
& =\int_{0}^{1} \frac{d}{d s}\left[P_{-s Y_{\theta}} t r_{g_{0}} \nabla_{c_{Y_{\theta}}^{\prime}}\left(\nabla^{g_{s t}}-\nabla^{g_{0}}\right) d\left(\exp s Y_{\theta}\right)\right] d s \\
& =\int_{0}^{1} P_{-s Y_{\theta}} \nabla_{c^{\prime}} t_{g_{0}} \nabla_{{c_{Y_{\theta}}^{\prime}}^{\prime}}\left(\nabla^{g_{s t}}-\nabla^{g_{0}}\right) d\left(\exp s Y_{\theta}\right) d s .
\end{aligned}
$$

But

$$
P_{-s Y_{\theta}} \nabla_{c^{\prime}} \operatorname{tr} r_{g_{0}} \nabla_{c_{Y_{\theta}}^{\prime}}\left(\nabla^{g_{s t}}-\nabla^{g_{0}}\right) d\left(\exp s Y_{\theta}\right)
$$

can be estimated similarly to

$$
P_{-s Y_{\theta}} \nabla_{c^{\prime}} t r_{g_{0}} \nabla_{c^{\prime}}\left(\nabla^{g_{s t}}-\nabla^{g_{0}}\right) d\left(\exp s Y_{\theta}\right),
$$

i.e. completely parallel to (7.47)-(7.55). One has only to replace $\theta Y$ by $Y_{\theta}$ and one of the $c^{\prime} s$ by $c_{Y_{\theta}}^{\prime}$. In our case here, $c^{\prime}=P_{s \theta Y} Y_{\theta}=P_{s \theta Y}\left(Y_{1}+\theta\left(Y_{2}-Y_{1}\right)\right)$, and $c_{Y_{\theta}}^{\prime}=$ $d\left(\exp s Y_{\theta}\right)\left(Y_{2}-Y_{1}\right)$. This has the consequence that in the polynomial $P_{r-2}$ of $(7.55)$ each monomial instead of a factor $|Y|_{r}^{2}$ now has a factor $\left(\left|Y_{1}\right|_{r}+\left|Y_{2}-Y_{1}\right|_{r}\right)\left|Y_{2}-Y_{1}\right|_{r}$. We will not repeat all details which are completely parallel to (7.47)-(7.54).

Hence,

$$
\begin{aligned}
& \left|P_{-Y_{\theta}} t r_{g_{0}} \nabla_{c_{Y_{\theta}}^{\prime}}\left(\nabla^{g_{t}}-\nabla^{g_{0}}\right) d\left(\exp Y_{\theta}\right)\right|_{r-2} \\
\leq & P_{r-2}\left(\left(\left|Y_{1}\right|_{r}+\left|Y_{2}-Y_{1}\right|_{r}\right),\left|Y_{2}-Y_{1}\right|_{r}\right)\left|Y_{2}-Y_{1}\right|_{r} \\
= & o(1)\left|Y_{2}-Y_{1}\right|_{r},
\end{aligned}
$$

where $o(1) \rightarrow 0$ as $\left|Y_{1}\right|_{r},\left|Y_{2}\right|_{r} \rightarrow 0, P_{r}=Q_{r} /\left|Y_{2}-Y_{1}\right|_{r}$, and $P_{r-2}$ are polynomials in the indicated variables with positive coefficients independent of $t_{0}$ and without constant term.

We infer from (7.124), (7.144), (7.154) and (7.156) that

$$
\begin{aligned}
|(7.61)|_{r-1} & =\left|P_{-Y_{\theta}} \nabla_{c_{Y_{\theta}}^{\prime}} \nabla^{g_{t}} d\left(\exp Y_{\theta}\right)\right|_{r-2} \\
& \leq o(1)\left|Y_{2}-Y_{1}\right|_{r},
\end{aligned}
$$

where $o(1) \rightarrow 0$ as $\left|Y_{1}\right|_{r},\left|Y_{2}\right|_{r} \rightarrow 0$.

The last step to prove Proposition 7.5 is to calculate and to estimate

$$
\lim _{\tau \rightarrow 0} \frac{1}{\tau}\left[P_{-\left(Y_{\theta}+\tau\left(Y_{2}-Y_{1}\right)\right)} P_{0 \rightarrow \tau}-P_{-Y_{\theta}}\right] X(0)
$$

which is the expression of (7.59).

We consider two curves, begining at $\exp s Y_{\theta}$, ending at $f_{t_{0}}$,

$$
\begin{gathered}
c_{1}(s)=\exp \left[-s P_{Y_{\theta}} Y_{\theta}=\left\{\exp s Y_{\theta}\right\}^{-1}, \quad 0 \leq s \leq 1,\right. \\
c_{2}(s)=\left\{\begin{array}{cc}
\exp \left(Y_{\theta}+s\left(Y_{2}-Y_{1}\right)\right), & 0 \leq s \leq \tau, \\
\exp -\frac{s-\tau}{1-\tau} P_{Y_{\theta}+\tau\left(Y_{2}-Y_{1}\right)}\left[Y_{\theta}+\tau\left(Y_{2}-Y_{1}\right)\right], & \tau \leq s \leq 1,
\end{array}\right.
\end{gathered}
$$


and the homotopy $c_{t}(s), 1 \leq t \leq 2$, connecting $c_{1}$ and $c_{2}$

$$
\begin{aligned}
& \exp \left(Y_{\theta}+(t-1) s\left(Y_{2}-Y_{1}\right)\right), \quad \text { when } \quad 0 \leq s \leq \tau, \\
c_{t}(s)= & \exp _{\exp Y_{\theta}+(t-1) \tau\left(Y_{2}-Y_{1}\right)}\left[-\frac{s-\tau}{1-\tau} P_{Y_{\theta}+(t-1) \tau\left(Y_{2}-Y_{1}\right)} Y_{\theta}\right. \\
& \left.+(t-1) \tau\left(Y_{2}-Y_{1}\right)\right], \quad \text { when } \quad \tau \leq s \leq 1 .
\end{aligned}
$$

Then for $t>1, s \rightarrow c_{t}(s)$ is not smooth at $s=\tau$.

We calculate the tangent vectors to $c_{t}(s)$,

$$
\begin{gathered}
\frac{\partial c_{t}(s)}{\partial s}=\left(d \exp \left(Y_{\theta}+(t-1) s\left(Y_{2}-Y_{1}\right)\right)(t-1)\left(Y_{2}-Y_{1}\right), \quad 0 \leq s \leq \tau^{-},\right. \\
\frac{\partial c_{t}(s)}{\partial s}=-\frac{1}{1-\tau} P_{-\frac{s-\tau}{1-\tau}\left(Y_{\theta}+(t-1) \tau\left(Y_{2}-Y_{1}\right)\right)} P_{Y_{\theta}+(t-1) \tau\left(Y_{2}-Y_{1}\right)}\left[Y_{\theta}+(t-1) \tau\left(Y_{2}-Y_{1}\right)\right] \\
\leq-\frac{1}{1-\tau} P_{1-\frac{s-\tau}{1-\tau}\left(Y_{\theta}+(t-1) \tau\left(Y_{2}-Y_{1}\right)\right)}\left[Y_{\theta}+(t-1) \tau\left(Y_{2}-Y_{1}\right)\right] \\
\quad \tau^{+} \leq s \leq 1 \\
\frac{\partial c_{t}(s)}{\partial t}=\left(\operatorname{dexp}\left(Y_{\theta}+(t-1) s\left(Y_{2}-Y_{1}\right)\right) s\left(Y_{2}-Y_{1}\right), \quad 0 \leq s \leq \tau^{-}\right.
\end{gathered}
$$

The calculation of $\frac{\partial c_{t}(s)}{\partial t}$ for $s>\tau$ is a little bit more complicated. We set $\sigma=\frac{s-\tau}{1-\tau}$. Then $s$ runs through $[\tau, 1]$ if and only if $\sigma$ runs through $[\delta, 1]$. With $\sigma=\frac{s-\tau}{1-\tau}$, the curve $\left\{c_{t}(\sigma)\right\}_{0 \leq \sigma \leq 1}$ for $s \geq \tau^{+}$is just the inverse curve to

$$
\left\{\tilde{c}_{t}(\tilde{\sigma})\right\}_{0 \leq \tilde{\sigma} \leq 1}=\left\{\exp \tilde{\sigma}\left(Y_{\theta}+(t-1) \tau\left(Y_{2}-Y_{1}\right)\right)\right\}_{0 \leq \tilde{\sigma} \leq 1},
$$

in particular

$$
\begin{gathered}
c_{t}(\sigma)=\tilde{c}_{t}(1-\tilde{\sigma})=\tilde{c}_{t}(\tilde{\sigma}), \quad 0^{+} \leq \sigma \leq 1 \\
\frac{\partial c_{t}(s)}{\partial s}=\frac{\partial c_{t}(\sigma)}{\partial \sigma} \frac{\partial \sigma}{\partial s}=\frac{\partial c_{t}(\sigma)}{\partial \sigma} \frac{1}{1-\tau}=\frac{\partial \tilde{c}_{t}(\tilde{\sigma})}{\partial \tilde{\sigma}} \frac{\partial \tilde{\sigma}}{\partial \sigma} \frac{\partial \sigma}{\partial s}=-\frac{\partial \tilde{c}_{t}(\tilde{\sigma})}{\partial \tilde{\sigma}} \frac{1}{1-\tau}
\end{gathered}
$$

and

$$
\begin{aligned}
\left.\frac{\partial c_{t}(s)}{\partial t}\right|_{\sigma} & = \pm\left.\frac{\partial \tilde{c}_{t}(\tilde{s})}{\partial t}\right|_{\tilde{\sigma}} \\
& =\frac{\partial}{\partial t}\left[\exp (1-\sigma)\left(Y_{\theta}+(t-1) \tau\left(Y_{2}-Y_{1}\right)\right)\right] \\
& =\left[\operatorname{dexp}\left((1-\sigma)\left(Y_{\theta}+(t-1) \tau\left(Y_{2}-Y_{1}\right)\right)\right] \tau\left(Y_{2}-Y_{1}\right)\right. \\
& =d \exp \left[\frac{1-s}{1-\tau}\left(Y_{\theta}+(t-1) \tau\left(Y_{2}-Y_{1}\right)\right] \tau\left(Y_{2}-Y_{1}\right)\right.
\end{aligned}
$$

Now we come back to (7.158). The main idea how to attack it is represented in [1], p.92-93. In our case, we have two curves beginning at $\exp Y_{\theta}$ and ending at $f_{t_{0}}$, namely $c_{1}$ and $c_{2}$, and we have our homotopy $c_{t}$ between them. Denote $X(s, t)=P_{c_{t}(s)} X(0)$ the vector field along the homotopy which is parallel along the paths $c_{t}$. Then we denote $X_{i}(s)=P_{c_{i}(s)} X(0)$ and get

$$
X(0, t)=X(0,1)=X(0,2)=X(0), \quad \nabla_{s} X(s, t)=0,
$$


where $\nabla_{s} \equiv \nabla_{\frac{\partial c}{\partial s}}=\nabla_{\frac{\partial c_{t}}{\partial s}}, \nabla_{t} \equiv \nabla_{\frac{\partial c_{t}}{\partial t}}$. (7.158) can be rewritten as

$$
\lim _{\tau \rightarrow 0} \frac{X_{2}(1)-X_{1}(1)}{\tau} \text {. }
$$

We compare the tangent vectors at $\tau^{+}, \tau^{-}$, to determine the jump:

$$
\begin{gathered}
\left.\frac{\partial c_{t}(s)}{\partial s}\right|_{s=\tau^{-}}=\left(\operatorname{dexp}\left(Y_{\theta}+(t-1) \tau\left(Y_{2}-Y_{1}\right)\right)\right)(t-1)\left(Y_{2}-Y_{1}\right)=\frac{J_{t}(\tau)}{\tau}, \\
\left.\frac{\partial c_{t}(s)}{\partial s}\right|_{s=\tau^{+}}=-\frac{1}{1-\tau} P_{Y_{\theta}+(t-1) \tau\left(Y_{2}-Y_{1}\right)}\left[Y_{\theta}+(t-1) \tau\left(Y_{2}-Y_{1}\right)\right] .
\end{gathered}
$$

We see, the jump is just the difference between the parallel displacement and the (Jacobi field) $/ \tau$. For the $t$ derivative, the situation is much better:

$$
\left.\frac{\partial c_{t}(s)}{\partial t}\right|_{s=\tau^{-}}=\left(\operatorname{dexp}\left(Y_{\theta}+(t-1) \tau\left(Y_{2}-Y_{1}\right)\right)\right) \tau\left(Y_{2}-Y_{1}\right)=\left.\frac{\partial c_{t}(s)}{\partial t}\right|_{s=\tau^{+}} .
$$

If $s \rightarrow c_{t}(s)$ would be $C^{1}$, then, using $\nabla_{t} X(0, t)=0, \nabla_{t} \nabla_{s} X(s, t)=0$, we obtain

$$
\begin{aligned}
\left|X_{2}(1)-X_{1}(1)\right|_{g_{0}, f_{t_{0}}(x)} & \leq \int_{1}^{2}\left|\nabla_{t} X(1, t)\right| d t \\
& \leq \int_{1}^{2} \int_{0}^{1}\left|\nabla_{s} \nabla_{t} X(s, t)\right| d s d t \\
& =\int_{1}^{2} \int_{0}^{1}\left|R\left(\frac{\partial c_{t}}{\partial s}, \frac{\partial c_{t}}{\partial t}\right) X(s, t)\right| d s d t
\end{aligned}
$$

which holds for an arbitrary vector bundle.

Unfortunately, for $t>1$ our $c_{t}(s)$ are not $C^{1}$, so we cannot apply this procedure immediately. There are two ways out from this situation. The first one is to take into account the jump and its consequences into all calculations and estimates. Using (7.24), one can calculate and estimate the jump of $X(s, t)$. The other way is to use a family of arbitrarily dense $C^{1}$-approximations $\left\{c_{t}^{\tau}(s)\right\}_{\tau \rightarrow 0}$ of $c_{t}$, which has the following properties:

(1) For $s \notin[\tau-\varepsilon(\tau), \tau+\varepsilon(\tau)], \varepsilon(\tau)=o(\tau)$ for $\tau \rightarrow 0$, there holds

$$
c_{t}^{\tau}(s)=c_{t}(s)
$$

$(2)$

$$
\int_{1}^{2} \int_{0}^{1} \operatorname{dist}\left(c_{t}^{\tau}(s), c_{t}(s)\right) d s d \tau=o(\tau), \quad \text { for } \quad \tau \rightarrow 0
$$

(3) In normal coordinates,

$$
\sup \left|\nabla^{i}\left(\frac{\partial c_{t}^{\tau}}{\partial s}-\frac{\partial c_{t}}{\partial s}\right)\right|=o(\tau), \quad 0 \leq i \leq 2
$$

and

$$
\int_{1}^{2} \int_{0}^{1}\left|\nabla^{i}\left(\frac{\partial c_{t}^{\tau}}{\partial s}-\frac{\partial c_{t}}{\partial s}\right)\right| d s d t=o(\tau), \quad 2 \leq i \leq r-2
$$


These properties imply by means of $(7.24)$

$$
\begin{array}{r}
\sup \left|\nabla^{i}\left(X(s, t)-X^{\tau}(s, t)\right)\right| d s d t=o(\tau), \quad 0 \leq i \leq 2, \\
\int_{1}^{2} \int_{0}^{1}\left|\nabla^{i}\left(X(s, t)-X^{\tau}(s, t)\right)\right| d s d t=o(\tau), \quad 2 \leq i \leq r-2 .
\end{array}
$$

where $X^{\tau}(s, t)=P_{c_{t}^{\tau}(s)} X(0)$.

The estimate of such approximations is a standard fact in differential topology and geometry and is already contained in [25]. In our case of bounded geometry, we can do this uniformly on $M$.

Now we can estimate

$$
\begin{aligned}
& \left|X_{2}(1)-X_{1}(1)\right| \\
\leq & \left|X_{2}(1)-X_{2}^{\tau}(1)\right|+\left|X_{2}^{\tau}(1)-X_{1}^{\tau}(1)\right|+\left|X_{1}^{\tau}(1)-X_{1}(1)\right| \\
\leq & \int_{1}^{2} \int_{0}^{1}\left|R\left(\frac{\partial c_{t}^{\tau}}{\partial s}, \frac{\partial c_{t}^{\tau}}{\partial t}\right) X^{\tau}(s, t)\right| d s d t+o(\tau) \\
\leq & \sqrt{2} \sup _{s, t}\left|\frac{\partial c_{t}^{\tau}}{\partial s}\right| \cdot\left|\sup _{s, t} \frac{\partial c_{t}^{\tau}}{\partial t}\right| \cdot\left|\sup _{s, t}\right| X^{\tau}(s, t) \mid+o(\tau) \\
\leq & \left.\sqrt{2} \sup _{s, t}\left(\left|\frac{\partial c_{t}^{\tau}}{\partial s}-\frac{\partial c_{t}}{\partial s}\right|+\left|\frac{\partial c_{t}}{\partial s}\right|\right) \cdot \sup _{s, t}\left|\frac{\partial c_{t}^{\tau}}{\partial t}-\frac{\partial c_{t}}{\partial t}\right|+\left|\frac{\partial c_{t}}{\partial t}\right|\right)|\cdot| X(0) \mid+o(\tau) \\
\leq & \sqrt{2} \sup _{s, t}\left(\left|\frac{\partial c_{t}}{\partial s}\right|+o(\tau)\right) \cdot \sup _{s, t}\left(\left|\frac{\partial c_{t}}{\partial t}\right|+o(\tau)\right) \cdot|X(0)|+o(\tau) \\
= & \sqrt{2} \sup _{s, t}\left|\frac{\partial c_{t}}{\partial s}\right| \cdot \sup _{s, t}\left|\frac{\partial c_{t}}{\partial t}\right| \cdot|X(0)|+o(\tau) \\
= & \sqrt{2}\left\{\operatorname { m a x } _ { s , t } \left\{\sup \mid\left(d \exp \left(Y_{\theta}+(t-1) s\left(Y_{2}-Y_{1}\right)\right)(t-1)\left(Y_{2}-Y_{1}\right) \mid,\right.\right.\right. \\
& \left.\sup _{t} \frac{1}{1-\tau}\left|Y_{\theta}+(t-1) \tau\left(Y_{2}-Y_{1}\right)\right|\right\} \cdot \max \left\{\sup _{s, t} \mid d \exp \left(Y_{\theta}+(t-1) s\right.\right. \\
& \left.\left.\left.\cdot\left(Y_{2}-Y_{1}\right)\right) s\left(Y_{2}-Y_{1}\right)\left|, \sup _{s, t}\right| d \exp \frac{1-s}{1-\tau}\left(Y_{\theta}+(t-1) \tau\left(Y_{2}-Y_{1}\right)\right) \tau\left(Y_{2}-Y_{1}\right) \mid\right\}\right\} \\
& \cdot|X(0)|+o(\tau) .
\end{aligned}
$$

If we estimate the numerator in (7.168) by (7.178), then we see that the $\tau$ in the denominator of (7.168) cancels out against the $s \leq \tau$ or $\tau$ in the terms of (7.178).

Moreover,

$$
\begin{gathered}
\lim _{\tau \rightarrow 0^{+}} \sup _{\substack{s \leq \tau \\
t}}\left|d \exp \left(Y_{\theta}+(t-1) s\left(Y_{2}-Y_{1}\right)\right)(t-1)\left(Y_{2}-Y_{1}\right)\right|=\left|\left(d \exp Y_{\theta}\right)\left(Y_{2}-Y_{1}\right)\right|, \\
\lim _{\tau \rightarrow 0^{+}}\left|Y_{\theta}+(t-1) \tau\left(Y_{2}-Y_{1}\right)\right|=\left|Y_{\theta}\right|
\end{gathered}
$$$$
\lim _{\tau \rightarrow 0^{+}} \sup _{\substack{s \leq \tau \\ t}}\left|\exp \left(Y_{\theta}+(t-1) s\left(Y_{2}-Y_{1}\right)\right)\left(Y_{2}-Y_{1}\right)\right|=\left|\left(\operatorname{dexp} Y_{\theta}\right)\left(Y_{2}-Y_{1}\right)\right|,
$$ 


$$
\begin{aligned}
& \lim _{\tau \rightarrow 0^{+}} \sup _{\tau \leq s \leq 1}\left|\left[\operatorname{dexp} \frac{1-s}{1-\tau}\left(Y_{\theta}+(t-1) \tau\left(Y_{2}-Y_{1}\right)\right)\right]\left(Y_{2}-Y_{1}\right)\right| \\
& =\sup _{0 \leq s \leq 1}\left|\left(\exp (1-s) Y_{\theta}\right)\left(Y_{2}-Y_{1}\right)\right| .
\end{aligned}
$$

We obtain

$$
\begin{aligned}
& \lim _{\tau \rightarrow 0} \frac{1}{\tau}\left[\left(P_{-Y_{\theta}+\tau\left(Y_{2}-Y_{1}\right)} P_{0 \rightarrow \tau}-P_{-Y_{\theta}}\right) X(0)\right] \\
& \leq C\left|Y_{2}-Y_{1}\right| \cdot\left|Y_{2}-Y_{1}\right|
\end{aligned}
$$

or

$$
\leq C\left(\left|Y_{1}\right|+\left|Y_{2}-Y_{1}\right|\right)\left|Y_{2}-Y_{1}\right|
$$

Let $Z \in T_{f_{t_{0}}} M^{2}$ be a unit vector, radially parallel transfered to all directions and consider, according to (7.171),

$$
\begin{aligned}
& \left|\nabla_{Z} P_{c_{2}(1)} X(0)-\nabla_{Z} P_{c_{1}(1)} X(0)\right| \\
& \leq \int_{1}^{2} \int_{0}^{1}\left|\nabla_{s} \nabla_{t}\left(\nabla_{Z} P_{c_{t}(s)} X(0)\right)\right| d s d t \\
& =\int_{1}^{2} \int_{0}^{1}\left|\nabla_{s} \nabla_{Z} \nabla_{t} P_{c_{t}(s)} X(0)+\nabla_{s} R\left(\frac{\partial c_{t}}{\partial t}, Z\right) P_{c_{t}(s)} X(0)\right| d s d t
\end{aligned}
$$

$=\int_{1}^{2} \int_{0}^{1}\left|\nabla_{Z} \nabla_{s} \nabla_{t} P_{c_{t}(s)} X(0)+R\left(\frac{\partial c_{t}}{\partial s}, Z\right) \nabla_{t} P_{c_{t}(s)} X(0)+\nabla_{s} R\left(\frac{\partial c_{t}}{\partial t}, Z\right) P_{c_{t}(s)} X(0)\right| d s d t$ $\leq \int_{1}^{2} \int_{0}^{1} \mid \nabla_{Z} R\left(\frac{\partial c_{t}}{\partial s}, \frac{\partial c_{t}}{\partial t} P_{c_{t}(s)} X(0)|+| R\left(\frac{\partial c_{t}}{\partial s}, Z\right) \nabla_{t} P_{c_{t}(s)} X(0) \mid\right.$

$+\left|\nabla_{s} R\left(\frac{\partial c_{t}}{\partial t}, Z\right) P_{c_{t}(s)} X(0)\right| d s d t$

i.e., we have to estimate

$$
\begin{gathered}
\int_{1}^{2} \int_{0}^{1} \sqrt{2}\left\{\left[\left|\nabla \frac{\partial c_{t}}{\partial s} \| \frac{\partial c_{t}}{\partial t}\right|+\left|\frac{\partial c_{t}}{\partial s}\right|\left|\nabla \frac{\partial c_{t}}{\partial t}\right|\right]|X(0)|+\left|\frac{\partial c_{t}}{\partial s} \| \frac{\partial c_{t}}{\partial t}\right|\right. \\
\left.\cdot\left(\nabla P_{c_{t}(s)}\right) X(0)+P_{c_{t}(s)} \nabla X(0) \mid\right\} d s d t \\
\left.+\int_{1}^{2} \int_{0}^{1} \sqrt{2}\left|\frac{\partial c_{t}}{\partial s}\left\|\frac{\partial c_{t}}{\partial t}\right\|\left(\nabla P_{c_{t}(s)}\right) X(0)+P_{c_{t}(s)} \nabla X(0)\right|\right\} d s d t \\
+\int_{1}^{2} \int_{0}^{1} \sqrt{2}\left|\frac{\partial c_{t}}{\partial s}\left\|\nabla \frac{\partial c_{t}}{\partial t}\right\| X(0)\right| d s d t
\end{gathered}
$$

We see from (7.181)-(7.183) that each term contains a factor of one of the following forms:

$$
\left|Y_{2}-Y_{1}\right| \cdot\left|Y_{2}-Y_{1}\right|
$$




$$
\begin{gathered}
\left(\left|Y_{1}\right|+\left|Y_{2}-Y_{1}\right|\right)\left|Y_{2}-Y_{1}\right|, \\
\left|\nabla\left(Y_{2}-Y_{1}\right)\right| \cdot\left|Y_{2}-Y_{1}\right|, \\
\left(\left|\nabla Y_{1}\right|+\left|\nabla\left(Y_{2}-Y_{1}\right)\right|\right)\left|Y_{2}-Y_{1}\right|, \\
\left(\left|Y_{1}\right|+\left|Y_{2}-Y_{1}\right|\right)\left|\nabla\left(Y_{2}-Y_{1}\right)\right|,
\end{gathered}
$$

assuming for a moment $c_{t}(s) \in C^{1}$.

Now it is visible and absolutely parallel to the procedure above how to handle the case

$$
\left|\nabla^{i} P_{c_{2}(1)} X(0)-\nabla^{i} P_{c_{1}(1)} X(0)\right|,
$$

1). one changes $\nabla^{i} \nabla_{s} \nabla_{t}$ to $\nabla_{s} \nabla_{t} \nabla^{i}$, thus producing curvature terms containing lower derivatives;

2 ). one distributes derivatives $\nabla^{j}$ as $\nabla^{j_{r}}$ of the single components of a term, according to the Leibniz rule.

Thus one obtains

$$
\begin{aligned}
\left|\nabla^{i} P_{c_{2}(1)} X(0)-\nabla^{i} P_{c_{1}(1)} X(0)\right| \leq & \tau Q_{i}\left(\left|Y_{1}\right|+\left|Y_{2}-Y_{1}\right|\right), \cdots,\left|\nabla^{i} Y_{1}\right|+\left|\nabla^{i}\left(Y_{2}-Y_{1}\right)\right|, \\
& \left.\left|Y_{2}-Y_{1}\right|, \cdots,\left|\nabla^{i}\left(Y_{2}-Y_{1}\right)\right|\right),
\end{aligned}
$$

where $Q_{i}$ is a polynomial in the indicated variables with positive coefficients independent of $t_{0}$ without monomials of degree not greater than one and such that each monomial contains a $\left|\nabla^{j}\left(Y_{2}-Y_{1}\right)\right|$ as a factor.

Applying the module structure theorem, taking the $L_{2}$-norm and summing up $\sum_{i=0}^{r-2}$, we get

$$
\begin{aligned}
\left|P_{c_{2}(1)} X(0)-P_{c_{1}(1)} X(0)\right|_{r-2} & \leq \tau P_{r}\left(\left(\left|Y_{1}\right|_{r}+\left|Y_{2}-Y_{1}\right|_{r}\right),\left|Y_{2}-Y_{1}\right|_{r}\right) \cdot\left|Y_{2}-Y_{1}\right|_{r} \\
& =\tau \cdot o(1) \cdot\left|Y_{2}-Y_{1}\right|_{r}
\end{aligned}
$$

where $o(1) \rightarrow 0$ as $\left|Y_{1}\right|_{r},\left|Y_{2}\right|_{r} \rightarrow 0, P_{r}$ is a polynomial in the indicated variables with positive coefficients independent of $t_{0}$ and without constant term.

Our $c_{t}(s)$ is not smooth enough, but we approximate by $c_{t}^{\tau}(s)$, use (7.172)-(7.177) and obtain

$$
\left|P_{c_{2}(1)} X(0)-P_{c_{1}(1)} X(0)\right|_{r-2} \leq \tau \cdot o(1) \cdot\left|Y_{2}-Y_{1}\right|_{r}+o(\tau),
$$

for $\tau \rightarrow 0$.

We infer from (7.157) and (7.191)

$$
\left|g\left(t, Y_{2}\right)-g\left(t, Y_{1}\right)\right|_{r-2} \leq o(1)\left|Y_{2}-Y_{1}\right|_{r}
$$

$o(1) \rightarrow 0\left(\left|Y_{1}\right|_{r},\left|Y_{2}\right|_{r} \rightarrow 0\right)$ with rate independent of $t_{0}$. This finishes the proof of Proposition 7.5. $\square$

REMARK. Our variation of the continuity method by reduction to a uniform Banach fixed point theorem requires more or less the same efforts like the classical continuity method. There we have to prove the closedness of $L$ in $[0,1]$. A sequence $\left(\exp _{\nu} Y_{\nu}\right)_{\nu}=\left(\exp _{f_{t_{0}}} t_{\nu} Y_{\nu}\right)_{\nu}=\left(\exp t_{\nu} Y_{\nu} \circ f_{t_{0}}\right)_{\nu}$ of harmonic maps with $t_{\nu} \rightarrow t^{*}$ is a Cauchy sequence if and only if $\left(Y_{\nu}\right)_{\nu}$ is a Cauchy sequence, i.e. $\left|Y_{\mu}-Y_{\nu}\right|_{r} \rightarrow 0$ $(\mu, \nu \rightarrow 0)$. If $f_{\nu} \rightarrow f^{*}$, then $f^{*}$ is harmonic if and only if $F\left(t^{*}, Y_{\nu}\right) \rightarrow 0$. The latter is equivalent with $T_{t^{*}} Y_{\nu} \rightarrow Y^{*}$. This can be controlled by our estimate (7.192). 


\section{Proof of the main theorem.}

THEOREM 8.1. Suppose $\left(M^{2}, g_{0}\right)$ be an open surface of constant curvature $K_{g_{0}} \equiv$ $-1, r_{i n j}\left(g_{0}\right)>0, g \in \operatorname{comp}\left(g_{0}\right)_{-1},\left\{g_{t}\right\}_{0 \leq t \leq 1}$ a smooth arc between $g_{0}$ and $g_{1}$ in $\operatorname{comp}\left(g_{0}\right)_{-1}$, and suppose inf $\sigma_{e}\left(\Delta\left(g_{0}\right)\right)>0$. Then there exists a unique smooth arc $\left\{f_{t}\right\}_{0 \leq t \leq 1}$ of harmonic diffeomorphisms $f_{t}:\left(M^{2}, g_{0}\right) \rightarrow\left(M^{2}, g_{t}\right)$ bounded homotopic to the identity.

Proof. We considered in section 7 an arc $\left\{f_{t}\right\}_{0 \leq t \leq 1}$ of harmonic maps $f_{t}$ : $\left(M^{2}, g_{0}\right) \rightarrow\left(M^{2}, g_{t}\right)$, with $f_{t}$ bounded homotopic to $i d: M \rightarrow M$, since it results from the composition of exponential maps $x \rightarrow \exp _{x} X_{i}$, the $X_{i}$ Sobolev vector fields. According to theorem 4.1 of [31], p.1025, each $f_{t}$ is uniquely determined hence the whole arc is uniquely determined.

We use the following

Proposition 8.2. Let $f$ be a harmonic map of degree one between surfaces of constant curvature -1 . Then the Jacobian of $f$ is everywhere positive. In particular, $f$ is locally a diffeomorphism.

Proof. There are several proofs. We refer to [16], [22], [24] and [26]. One can infer from these proofs a proof for the case $M^{2}$ open. Denote by $J(f)$ the Jacobian determinant of $\mathrm{f}$.

LEMma 8.3. Under our assumptions, $J(f) \geq 0$.

Proof. This has been proven in [26], Lemma B.32, p. 182 or in [24], pp.270-271 by purely local methods like the local maximum principle.

We recall Proposition 2.2 from [24] as

LEMma 8.4. Let $\Omega \subset\left(M, g_{0}\right)$ be open, connected and $f: \Omega \rightarrow\left(M, g_{1}\right)$ be a harmonic map satisfying $J(f) \geq 0$. Then either $J(f)$ is identically zero or all zeros of $J(f)$ are isolated. Moreover, if there is a number $k$ so that $\# f^{-1}(q) \leq k$ for each regular value $q \in\left(M, g_{1}\right)$ of $f$, then each isolated zero of $J(f)$ is a non-trivial branch point of $f$.

Now let $\Omega \subset\left(M, g_{0}\right)$ be as above and relative compact. According to Lemma 8.4, isolated zeros of $J(f)$ correspond to non-trivial branch points of $f$. But we have degree $(f)=1$, hence such branch points do not exist, $J(f)>0$ on $\Omega$ and hence on all of $M$.

REMARK. For open manifolds there exists a well-defined fundamental cycle in locally finite bounded homology. Hence the notion of mapping degree one is welldefined.

Back to the proof of Theorem 8.1. In our case, any map $x \rightarrow \exp _{x} X$ is proper, since the preimage of any compact set $K \subset M$ is a closed subset of $\frac{\mathscr{U}_{r_{i n j}}(K)}{}$. The finite composition of proper maps is also proper. Hence $f$ is proper, and a finite covering of itself. Since degree $(f)=1$, there is only one leaf, $f$ is a diffeomorphism and the whole curve $\left\{f_{t}\right\}_{0 \leq t \leq 1}$ consists of harmonic diffeomorphisms. 


\section{REFERENCES}

[1] P. Buser And H. Karcher, Gromov's almost flat manifolds. Astérisque, 81. Paris: Societé Mathématique de France; 1981.

[2] S. Y. Cheng, Liouville theorem for harmonic maps, Proc. Sympos. Pure Math., 36 (1980), pp. $147-151$.

[3] W. Y. Ding AND Y. D. WANG, Harmonic maps of complete noncompact Riemannian manifolds, Intern. J. Math., 2 (1991), pp. 617-633.

[4] J. Eells and L. Lemaire, Two reports on harmonic maps, World Scientific Publishing Co., Inc., River Edge, NJ, 1995.

[5] J. EichHorn, Poincare's theorem and Teichmüller theory for open surfaces, Asian J. Math., 2 (1998), pp. 355-404

[6] J. EichHorn, The manifold structure of maps between open manifolds, Ann. Global Anal. Geom., 11:3 (1993), pp. 253-300.

[7] J. EichHorn, A slice theorem for open manifolds, Geom. Dedicata, 91 (2002), pp. 85-116.

[8] J. EichHoRn, Spaces of Riemannian metrics on open manifolds, Results Math., 27 (1995), pp. 256-283.

[9] J. EichHORN, Uniform structures of metric spaces and open manifolds, Results Math0., 40 (2001), pp. 144-191.

[10] J. EichHORN, The boundedness of connection coefficients and their derivatives, Math. Nachr., 153 (1991), pp. 145-158.

[11] J. EichHORN AND J. Fricke, The module structure theorem for Sobolev spaces on open manifolds, Math. Nachr., 194 (1998), pp. 35-47.

[12] J. EichHorn, Global analysis on open manifolds, Nova Science Publ., 664pp, New York 2007.

[13] D. Freed And D. Groisser, The basic geometry of the manifold of Riemannian metrics and its quotient by the diffeomorphism group, Mich. Math. J., 36 (1989), pp. 324-344.

[14] D. Gilbarg And N. Trudinger, Elliptic partial differential equations of second order, Springer, 2001.

[15] J. Jost, Nonlinear methods in Riemannian and Kählerian geometry, Birkhüser Verlag, 1988.

[16] J. Jost, Two-dimensional geometric variational problems, Wiley Interscience Publ., New York, 1990.

[17] J. Y. LI, The heat flows and harmonic maps of complete noncompact Riemannian manifolds, Math. Z., 212 (1993), pp. 161-173.

[18] M. LI, Harmonic maps equations on noncompact Riemannian manifolds, Suplems Sci. Math., 13 (2000), pp. 333-336.

[19] P. Li AND L. F. TAM, The heat equation and harmonic maps on complete manifolds, Invent. Math., 105 (1991), pp. 1-46.

[20] J. LohkAmp, An existence theorem for harmonic maps, Manuscripta Math., 67 (1990), pp. 2123.

[21] J. Lohkamp, Harmonic diffeomorphisms and Teichmüller theory, Manuscripta Math., 71 (1991), pp. 339-360.

[22] J. H. SAmpson, Some properties and applications of harmonic mappings, Ann. Scient. Ec. Norm. Sup., 11 (1978), pp. 211-228.

[23] R. Schoen And S.-T. YAU, Compact group actions and the topology of manifolds with nonpositive curvature, Topology, 18 (1979), pp. 361-380.

[24] R. Schoen And S.-T. Yau, On univalent harmonic maps between surfaces, Invent. math., 44 (1978), pp. 265-278.

[25] N. E. Steenrod, Topology of fibre bundles, Princeton University Press, Princeton 1951.

[26] A. Tromba, Teichmüller theory in Riemannian geometry, Birkhüser Verlag, Basel 1992.

[27] Y. D. WANG, Harmonic maps from complete noncompact Riemannian manifolds, J. Part. Diff. Equs., 12 (1999), pp. 262-280.

[28] J. WlokA, Partielle Differentialgleichungen, B. G. Teubner, Stuttgart, 1982.

[29] M. Wolf, Measured foliations and harmonic maps of surfaces, J. Differential Geom., 49:3 (1998), pp. 437-467.

[30] M. Weber AND M. Wolf, Teichmüller theory and handle addition for minimal surfaces, Ann. of Math. (2), 156:3 (2002), pp. 713-795.

[31] D. YANG, Deforming a map into a harmonic map, Trans. AMS., 352 (1999), pp. 1021-1038.

[32] S.-T. YAU, Some function-theoretic properties of complete Riemannian manifolds and their applications to geometry, Indiana Univ. Math. J., 25 (1976). 
Q. CHEN AND J. EICHHORN 